Estimação e diagnóstico na disribuição WeibullBinomial-Negativa em análise de sobrevivência 


\title{
Estimação e diagnóstico na disribuição Weibull-Binomial- Negativa em análise de sobrevivência
}

\author{
Bao Yiqi \\ Orientador: Prof. Dr. Vicente Garibay Cancho \\ Dissertação apresentada ao Instituto de Ciências \\ Matemáticas e de Computação - ICMC-USP, como parte \\ dos requisitos para obtenção do título de Mestre em \\ Ciências - Ciências de Computação e Matemática \\ Computacional. VERSÃO REVISADA
}

USP - São Carlos

Junho de 2012 
Ficha catalográfica elaborada pela Biblioteca Prof. Achille Bassi e Seção Técnica de Informática, ICMC/USP, com os dados fornecidos pelo(a) autor(a)

B221e Bao, Yiqi Binomial-Negativa em análise de sobrevivência / Yiqi Bao; orientador Vicente Garibay Cancho. -- São Carlos, 2012.

$95 \mathrm{p}$.

Dissertação (Mestrado - Programa de Pós-Graduação en Ciências de Computação e Matemática Computacional) -Instituto de Ciências Matemáticas e de Computação, Universidade de São Paulo, 2012.

1. análise de sobrevivência. 2. distribuição Binomial Negativa. 3. distribuição Weibull. 4. inferência Bayesiana. 5. modelos de sobrevivência de fração de cura. I. Cancho, Vicente Garibay, orient. II. Título. 
Aos meus pais, Bao Ke Wei e Wang Cuiqin, pelo incentivo e esforço para minha formação.

Aos meus amigos do laboratório de estatística, pela amizade e apoio. 


\section{Agradecimentos}

Primeiramente agradeço a Deus por tudo, pela minha saúde, minha família, e por ter me dado a oportunidade desta conquista.

Ao meu orientador, Vicente Garibay Cancho, pela orientação, sugestões, paciência e amizade que contribuíram para o meu crescimento e minha formação acadêmica.

Aos meus pais que sempre me ajudam, apoiam e incentivam, e a quem sempre estarei em dívida.

Aos professores Francisco Louzada Neto, Heleno Bolfarine e à professora Juliana Cobre, pelas sugestões.

Aos professores e funcionários da biblioteca Prof. Achille Bassi e da pós-graduação do ICMC, pelo excelente convívio.

A todos os meus amigos do ICMC, Ariadne, Alina, Aline, Daiane, Guto, Mateus e Willian pelos momentos compartilhados durante a minha formação.

A todas as pessoas que não foram nominalmente mencionadas, mas que de alguma forma contribuíram para viabilizar este trabalho.

Finalmente, agradeço à Fundação de Amparo à Pesquisa do Estado de São Paulo (FAPESP) pelo suporte financeiro concedido para a realização deste trabalho. 


\section{Resumo}

Neste trabalho propomos a distribuição Weibull-Binomial-Negativa (WBN) considerando uma estrutura de ativação latente para explicar a ocorrência do evento de interesse, em que o número de causas competitivas é modelado pela distribuição Binomial Negativa, e os tempos não observados devido às causas seguem a distribuição Weibull. Em geral, as causas competitivas podem ter diferentes mecanismos de ativação, sendo assim os casos de primeira ativação, última ativação e ativação aleatória foram considerados no estudo. Desse modo o modelo proposto inclui uma ampla distribuição, tais como Weibull-Geométrico (WG) e Exponencial-Poisson Complementar (EPC), introduzidas por Barreto-Souza et al. (2011) e G. et al. (2011), respectivamente. Baseando-nos na mesma estrutura, consideramos o modelo de regressão locação-escala baseado na distribuição proposta (WBN) e o modelo para dados de sobrevivência com fração de cura. Os principais objetivos deste trabalho é estudar as propriedades matemáticas dos modelos propostos e desenvolver procedimentos de inferências desde uma perspectiva clássica e Bayesiana. Além disso, as medidas de diagnóstico Bayesiana baseadas na $\psi$-divergência (Peng \& Dey, 1995; Weiss, 1996), que inclui como caso particular a medida de divergência Kullback-Leibler (K-L), foram consideradas para detectar observações influentes.

Palavras-chave: análise de sobrevivência, distribuição Binomial Negativa, distribuição Weibull, inferência Bayesiana, modelos de sobrevivência de fração de cura. 


\section{Abstract}

In this work we propose the Weibull-Negative-Binomial (WNB) considering a latent activation structure to explain the occurrence of an event of interest, where the number of competing causes are modeled by the Negative Binomial distribution and the no observed time due to the causes following the Weibull distribution. In general, the competitive causes may have different activation mechanisms, cases of first, last and random activation were considered in the study. Thus, the proposed model includes a wide distribution such as Weibull-Geometric distribution (WG) and Exponential-Poisson complementary (EPC) introduced by (Barreto-Souza et al., 2011) and (G. et al., 2011) respectively. Based on the same structure, we propose a location-scale regression model based on the proposed distribution (WNB) and the model for survival data with cure fraction. The main objectives of this work is to study the mathematical properties of the proposed models and develop procedures inferences from a classical and Bayesian perspective. Moreover, the Bayesian diagnostic measures based on the $\psi$-divergence (Peng \& Dey, 1995; Weiss, 1996), which includes Kullback-Leibler (K-L) divergence measure as a particular case, were considered to detect influential observations.

Keywords: Bayesian inference, negative binomial distribution, survival analysis, survival models with a cure fraction, Weibull distribution. 


\section{Lista de Figuras}

1.1 (a) Função de sobrevivência e (b) Função de risco da distribuição Weibull com $\lambda=0$ e diferentes valores de $\alpha$ selecionados . . . . . . . . . . . . . 4

2.1 (a)Função de sobrevivência; (b)Função de risco da distribuição WBN com parâmetros $\lambda=0, \alpha=1,5, \eta=10$ e $\theta=2 \ldots \ldots \ldots$

2.2 Função densidade de probabilidade da distribuição WBN-PA para alguns valores selecionados . . . . . . . . . . . . . . . . . . 17

2.3 Função de risco da distribuição WBN-PA para alguns valores selecionados .

2.4 Função densidade de probabilidade da distribuição WBN-UA para alguns valores selecionados ......................... 24

2.5 Função de risco da distribuição WBN-UA para alguns valores selecionados 26

2.6 Figura da função densidade de probabilidade e função densidade acumulada dos modelos ajustados . . . . . . . . . . . . . . . 37

2.7 Figura da função densidade de probabilidade e função densidade acumulada dos quatro melhores modelos ajustados . . . . . . . . . . . .

3.1 Funções de (a) densidade probabilidade e (b) risco da distribuição LWBN-PA para alguns parâmetros selecionados . . . . . . . . . . . . .

3.2 Funções de (a) densidade probabilidade e (b) risco da distribuição LWBNUA para alguns parâmetros selecionados . . . . . . . . . . . . 
3.3 Estimativas de Monte Carlo das medidas de $\psi$-divergência do modelo de regressão LWBN-PA ajustado. Painel superior esquerdo: Configuração A, conjunto de dados sem perturbação. Painel superior direito: Configuração B, conjunto de dados com observação 45 perturbada. Painel inferior esquerdo: Configuração C, conjunto de dados com observação 350 perturbada. Painel inferior direito: Configuração D, conjunto de dados com observações 45 e 350 perturbadas. . . . . . . . . . . . . . . .

3.4 Estimativas de Monte Carlo das medidas de $\psi$-divergência do modelo de regressão LWBN-UA ajustado. Painel superior esquerdo: Configuração A, conjunto de dados sem perturbação. Painel superior direito: Configuração B, conjunto de dados com observação 45 perturbada. Painel inferior esquerdo: Configuração C, conjunto de dados com observação 350 perturbada. Painel inferior direito: Configuração D, conjunto de dados com observações 45 e 350 perturbadas. . . . . . . . . . . . . . . . .

3.5 Dados originais. Painel esquerdo: Função densidade acumulada ajustada pelo modelo de regressão LWBN-PA. Painel direito: Estimativas de Monte Carlo das medidas de $\psi$-divergência do modelo de regressão LWBN-PA

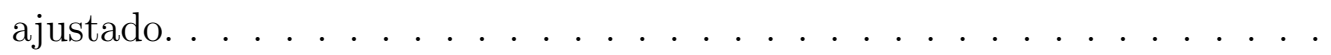

3.6 Dados reais sem observações 686 e 687. Painel esquerdo: Função densidade acumulada ajustada pelo modelo de regressão LWBN-PA. Painel direito: Estimativas de Monte Carlo das medidas de $\psi$-divergência do modelo de regressão LWBN-PA ajustado. . . . . . . . . . . . . . . . .

4.1 Função de sobrevivência da distribuição WBN com fração de cura sobre três diferentes esquemas de ativação com parâmetros $\lambda=0, \alpha=1.5, \eta=3 \mathrm{e}$

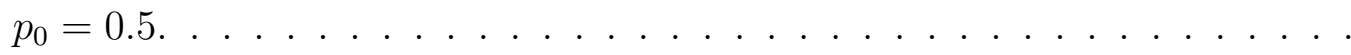


4.2 Estimativas de Monte Carlo das medidas de $\psi$-divergência do modelo de regressão WBNcr-PA ajustado. Painel superior esquerdo: Configuração A, conjunto de dados sem perturbação. Painel superior direito: Configuração B, conjunto de dados com observação 18 perturbada. Painel inferior esquerdo: Configuração C, conjunto de dados com observação 300 perturbada. Painel inferior direito: Configuração D, conjunto de dados com observações 18 e 300 perturbadas. . . . . . . . . . . . . . . . . .

4.3 Estimativas de Monte Carlo das medidas de $\psi$-divergência do modelo de regressão WBNcr-UA ajustado. Painel superior esquerdo: Configuração A, conjunto de dados sem perturbação. Painel superior direito: Configuração B, conjunto de dados com observação 18 perturbada. Painel inferior esquerdo: Configuração C, conjunto de dados com observação 300 perturbada. Painel inferior direito: Configuração D, conjunto de dados com observações 18 e 300 perturbadas. . . . . . . . . . . . . . . . . .

4.4 Estimativas de Monte Carlo das medidas de $\psi$-divergência do modelo de regressão WBNcr-AA ajustado. Painel superior esquerdo: Configuração A, conjunto de dados sem perturbação. Painel superior direito: Configuração B, conjunto de dados com observação 18 perturbada. Painel inferior esquerdo: Configuração C, conjunto de dados com observação 300 perturbada. Painel inferior direito: Configuração D, conjunto de dados com observações 18 e 300 perturbadas. . . . . . . . . . . . . . . . .

4.5 Painel esquerdo: Estimativas de Kaplan-Meier da função de sobrevivência. Painel direito: Gráfico QQ dos resíduos quantis aleatorizados e normalizados do modelo ajustado WBNcr-PA . . . . . . . . . . . . . . .

4.6 Estimativas de Kaplan-Meier e paramétricas da função de sobrevivência de acordo com a categoria nódulo $(1$ a 4$)$. . . . . . . . . . . . . .

4.7 Estimativas de Monte Carlo das medidas de $\psi$-divergência do modelo de regressão WBNcr-PA ajustado para conjunto de dados original . . . . . . . 
4.8 Estimativas de Monte Carlo das medidas de $\psi$-divergência do modelo de regressão WBNcr-PA ajustado sem covariáveis não significantes e observação 23,176 e $279 \ldots \ldots \ldots \ldots \ldots \ldots$

4.9 (a): Estimativas de Kaplan-Meier e paramétrica da função de sobrevivência do modelo de regressão WBNcr-PA de acordo com a categoria de nódulo (1-4) e (b): Funções de sobrevivência ajustadas estratificadas por nódulo para o paciente sem fração de cura . . . . . . . . . . . . . . . 


\section{Lista de Tabelas}

2.1 Momento ordinário, variância, assimetria e curtose da distribuição WBN-PA para alguns valores selecionados . . . . . . . . . . . . . . . . 21

2.2 Momentos, variância, assimetria e curtose das estatísticas de ordem WBNPA para alguns valores selecionados . . . . . . . . . . . . . . . . 23

2.3 Momento ordinário, variância, assimetria e curtose da distribuição WBN-UA para alguns valores selecionados . . . . . . . . . . . . . . . 27

2.4 Momentos, variância, assimetria e curtose das estatísticas de ordem da distribuição WBN-UA para alguns valores selecionados . . . . . . . . . . . 29

2.5 Estimativas da função de sobrevivência na mediana, EP e RQEM . . . . . 35

2.6 Porcentagem de amostras em que o modelo ajustado foi indicado como o melhor modelo de acordo com o critério AIC . . . . . . . . . . . . . . 35

2.7 AIC e SBC para os modelos ajustados . . . . . . . . . . . . . . 36

2.8 Frequências observadas e esperadas dos números de adultos de Tribolium confusum cultivados em $29^{\circ} \mathrm{C}$ e DMA entre as frequências . . . . . . . . . 37

2.9 Estimativas de função de verossimilhança e valor AIC para as distribuições ajustadas. . . . . . . . . . . . . . . . . . 38

2.10 As estimativas de máxima verossimilhança dos parâmetros para os modelos WBN-UA, WG-UA, WP-UA e Weibull . . . . . . . . . . . . . . 38

3.1 Estimativas dos critérios Bayesianos dos modelos ajustados para os dados

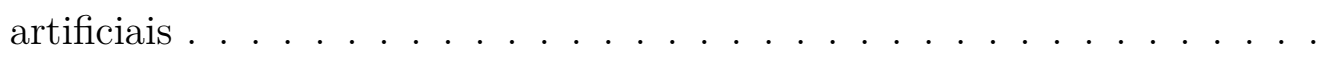


3.2 Estimativas da função de sobrevivência na mediana, EP e RQEM para os dados artificiais . . . . . . . . . . . . . . . . . . 48

3.3 Estimativas da função de sobrevivência na mediana, EP e RQEM para os dados artificiais ............................ 50

3.4 Estimativas dos critérios Bayesianos dos modelos ajustados para os dados

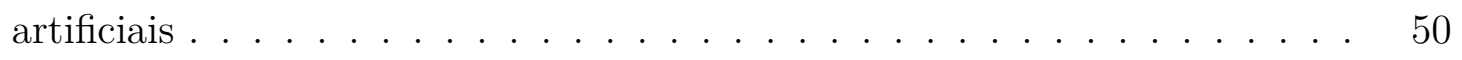

3.5 Estimativas das medidas de $\psi$-divergência para os dados artificiais. . . . . 51

3.6 Conjunto de dados reais. Critérios Bayesianos para modelos selecionados. . 54

3.7 Conjunto de dados reais. Resumo a posteriori dos parâmetros do modelo de regressão LWBN-PA. . . . . . . . . . . . . . . . . . . 54

3.8 Conjunto de dados reais. Resumo a posteriori dos parâmetros do modelo de regressão LWBN-PA. . . . . . . . . . . . . . . . . . .

4.1 Função de Sobrevivência $S_{\text {pop }}(y)$ e $f d p f_{\text {pop }}(y)$ para o modelo WBN com fração de cura sobre três diferentes esquemas de ativação . . . . . . . . . .

4.2 Porcentagem de amostras em que o modelo ajustado foi indicado como o melhor modelo de acordo com o critério AIC . . . . . . . . . . . . . . 69

4.3 EMV, EP e RQEM das frações de cura $\left(p_{0}^{(0)}\right.$ e $\left.p_{0}^{(1)}\right)$ para os dados simulados 70

4.4 Estimativas dos critérios Bayesianos dos modelos ajustados para os dados artificiais . . . . . . . . . . . . . . . . . 73

4.5 Estimativas das frações de cura, EP e RQEM para os dados artificiais . . . 73

4.6 Estimativas das frações de cura, EP e RQEM para os dados artificiais . . . 75

4.7 Estimativas dos critérios Bayesianos dos modelos ajustados para os dados artificiais ............................ 76

4.8 Estimativas das medidas de $\psi$-divergência para os dados artificiais. . . . . . 77

4.9 Critérios AIC e SBC para os modelos ajustados . . . . . . . . . . . . 82

4.10 Estimativas dos EMV dos parâmetros para o modelo WBNcr-PA . . . . . . 82 
4.11 Estimativas dos EMV dos parâmetros para o modelo WBNcr-PA . . . . . . 83

4.12 Resumo da fração de cura estratificada por categoria do nódulo. . . . . . . . 83

4.13 Conjunto de dados reais. Critérios Bayesianos para modelos selecionados. . 84

4.14 Conjunto de dados reais. Resumo a posteriori dos parâmetros para o modelo de regressão WBNcr-PA. . . . . . . . . . . . . . . . 84

4.15 Possíveis observações influentes detectadas por $\psi$-divergence . . . . . . . . 85

4.16 Resumo a posteriori dos parâmetros do modelo de regressão WBNcr-PA para dados reais . . . . . . . . . . . . . . 86

4.17 Resumo a posteriori dos parâmetros do modelo de regressão WBNcr-PA para dados reais com covariável significante . . . . . . . . . . . . 87

4.18 Resumo a posteriori das frações de cura de acordo com categoria de nódulo 88 


\section{Sumário}

1 Introdução $\ldots \ldots \ldots \ldots \ldots \ldots \ldots \ldots \ldots \ldots \ldots$

1.1 Caracterização da distribuição de tempo de vida . . . . . . . . . . . . . . . 3

1.2 Distribuição de Weibull . . . . . . . . . . . . . . . . . . . 3

1.2.1 Quantis e momentos .................. 4

1.3 Critérios de comparação de modelos . . . . . . . . . . . . . . . 5

1.4 Método de diagnóstico . . . . . . . . . . . . . . . 6

1.4.1 Análise de influência caso a caso . . . . . . . . . . . . . . 7

1.5 Organização dos capítulos . . . . . . . . . . . . . . . 10

2 Modelo Weibull-Binomial-Negativo . . . . . . . . . . . 11

2.1 Introdução . . . . . . . . . . . . . . . . . . . . . . 11

2.2 Formulação do modelo . . . . . . . . . . . . . . . . . . . . . . 12

2.2.1 A distribuição WBN sob esquema de primeira ativação . . . . . . . 16

2.2.2 A distribuição WBN sob esquema de última ativação . . . . . . . . . 24

2.3 Inferência Clássica . . . . . . . . . . . . . . . . . . . . . . . 29

2.4 Simulação . . . . . . . . . . . . . . . . . . . . 34

2.5 Aplicação . . . . . . . . . . . . . . . . . . . 36

2.6 Conclusão . . . . . . . . . . . . . . . . . . . . . . 39 
3 Modelo de regressão de Log-Weibull-Binomial-Negativo . . . . . . . . . 40

3.1 Introdução . . . . . . . . . . . . . . . . . . . . . . . . . 40

3.2 Formulação do modelo de regressão LWBN . . . . . . . . . . . . . . . . . . 41

3.3 Inferência Bayesiana . . . . . . . . . . . . . . . . . . . . . . 45

3.4 Estudo de dados artificiais . . . . . . . . . . . . . . . . . . . 47

3.4.1 Análise de influência . . . . . . . . . . . . . . . . . . . . 49

3.5 Aplicação . . . . . . . . . . . . . . . . . . . . . . . . 54

3.6 Conclusão . . . . . . . . . . . . . . . . . . . . . . . . . 56

4 Modelo de sobrevivência com fração de cura . . . . . . . . . . . . 57

4.1 Introdução . . . . . . . . . . . . . . . . . . . . . . . . . 57

4.2 Formulação do modelo . . . . . . . . . . . . . . . . . . . . . . . 58

4.3 Inferência Clássica . . . . . . . . . . . . . . . . . . . . . . 64

4.4 Estudo de simulação . . . . . . . . . . . . . . . . . . . . . . . . 67

4.5 Inferência Bayesiana . . . . . . . . . . . . . . . . . . . 70

4.6 Estudo de dados artificiais . . . . . . . . . . . . . . . . . . . 72

4.6 .1 Análise de influência . . . . . . . . . . . . . . . . . . . . . . . 73

4.7 Aplicação . . . . . . . . . . . . . . . . . . . . . . . 81

4.7.1 Análise Clássica . . . . . . . . . . . . . . . . . . . . . 81

4.7.2 Análise Bayesiana . . . . . . . . . . . . . . . . . . . . . . 84

4.8 Conclusão . . . . . . . . . . . . . . . . . . . . . . . . . . . . . . . . 89

5 Considerações finais . . . . . . . . . . . . . . . . . 90

Referências Bibliográficas ................... 92 


\section{Capítulo 1}

\section{Introdução}

A análise de sobrevivência é formada pelo conjunto de técnicas estatísticas para analisar dados correspondentes ao tempo até a ocorrência de um determinado evento de interesse, o qual é denominado tempo de sobrevivência ou tempo de falha. Por exemplo: tempo de vida até morte de um paciente com uma doença previamente diagnosticada; tempo até a falha de um componente eletrônico; ou tempo até a cura de um paciente após tratamento adequado. É comum em análise de sobrevivência a presença de observações parciais ou incompletas, ou seja, observações que não apresentam o evento de interesse, as quais são denominadas observações censuradas, podendo ocorrer por uma variedade de razões:

(i) A perda do acompanhamento do paciente no decorrer do estudo;

(ii) A não ocorrência do evento de interesse até o término do experimento;

(iii) O término do experimento antes da ocorrência do evento de interesse.

Os tempos de censuras devem ser incorporados às análises estatísticas, pois informam que até aquele instante o indivíduo estava vivo, e a sua omissão pode acarretar conclusões viciadas. As censuras são classificadas em tipo I, tipo II e aleatória. Mais detalhes podem ser encontrados em Colosimo \& Giolo (2006).

Em muitas situações a ocorrência de um evento de interesse pode ser devido a uma dentre várias causas competitivas; tanto o número de causas como o tempo de sobrevivência associado à cada causa, em geral, não são observados. Quando a probabilidade do número 
de causas competitivas for igual a zero e ela for não nula, temos que os dados de tempos de vida observados apresentarão uma fração de indivíduos para os quais o evento de interesse não ocorre; desse modo, esses tipos de dados podem ser acomodados por modelos de sobrevivência com fração de cura. Considerando diferentes distribuições para o número de causas competitivas e para os tempos de vida, muitos modelos de sobrevivência com fração de cura tem sido propostos na literatura (veja, Maller \& Zhou (1996); Ibrahim et al. (2001); Tsodikov et al. (2003); Cooner et al. (2007); Tournoud \& Ecochard (2007); Lopes et al. (2012); Rodrigues et al. (2009); Cancho et al. (2009); Cancho et al. (2011); Rodrigues et al. (2010a); Rodrigues et al. (2010b)). No entanto, quando a probabilidade do número de causas competitivas for igual a zero e ela for nula, ou seja, se existe pelo menos uma causa com probabilidade diferente de zero, e considerando que a ordem de ativação das causas competitivas é aleatória ou fixa, uma nova classe de família de distribuição pode ser obtida.

O objetivo deste trabalho é propor modelos para dados de tempo de vida com (ou sem) fração de cura com causas competitivas e diferentes mecanismos de ativação.

Entretanto, quando se está ajustando um modelo a um conjunto de dados, é imprescindível que as estimativas obtidas a partir do modelo proposto sejam resistentes a pequenas pertubações nas observações ou no modelo, pois se o modelo ajustado não apresentar uma boa descrição dos dados que foram observados, poderá conduzir a inferências errôneas. Assim sendo, é importante que seja realizado um estudo sobre a robustez dos resultados obtidos com relação aos vários aspectos que envolvem a formulação do modelo e as estimativas dos seus parâmetros, através de uma análise de diagnóstico, formada por métodos para avaliar o grau de sensibilidade das inferências a perturbações nos dados ou mesmo no modelo proposto.

Neste trabalho discutimos a influência do diagnóstico em uma abordagem Bayesiana e propomos as medidas de diagnóstico baseadas na $\psi$-divergência (Peng \& Dey, 1995; Weiss, 1996), que inclui como caso particular a medida de divergência Kullback-Leibler (K-L). 


\subsection{Caracterização da distribuição de tempo de vida}

A análise estatística de tempo de vida é um tópico amplamente utilizado em diversas áreas, tais como medicina, biologia, epidemiologia, engenharia, entre outros. O tempo de falha se refere ao tempo até a ocorrência de um evento de interesse, podendo ser a morte, o aparecimento de um tumor, o desenvolvimento de uma doença, a quebra de um componente eletrônico e assim por diante.

O tempo de falha, denotado por $T$, é uma variável aleatória não negativa, caracterizada através da função de sobrevivência, definida como:

$$
S(t)=P[T \geq t]=1-F(t)=\int_{t}^{\infty} f(t) d t
$$

que é a probabilidade de um indivíduo sobreviver até o tempo $t$, em que $f(t)$ é a função densidade de probabilidade $(f d p), F(t)$ é a função densidade acumulada $(f d a)$ e $S(t)$ é uma função monótona decrescente contínua com $S(0)=1$ e $\lim _{t \rightarrow \infty} S(t)=0$.

Outra função importante é a função de taxa de falha (ou função de risco), a qual especifica a taxa instantânea de falha ou morte de um indivíduo no tempo $t$, dado que ele sobreviveu até o tempo $t$, ou seja, é útil para descrever a distribuição do tempo de vida da observação em estudo, e que é definida por:

$$
h(t)=\lim _{\delta t \rightarrow 0} \frac{P[t \leq T \leq t+\delta t \mid T \geq t]}{\delta t}=\frac{f(t)}{S(t)} .
$$

\subsection{Distribuição de Weibull}

Seja $T$ uma variável aleatória não negativa que denota o tempo de vida de um indivíduo em uma população, dizemos que $T$ segue uma distribuição Weibull com parâmetro de forma $\alpha>0$ e parâmetro de escala $e^{\lambda}, \lambda \in \mathbb{R}$, denotada por $T \sim W \operatorname{eibull}(\alpha, \lambda)$, e sua função densidade de probabilidade $(p d f)$ é dada por

$$
f(t)=\alpha t^{\alpha-1} \exp \left(\lambda-t^{\alpha} e^{\lambda}\right)
$$


A função densidade acumulada $(f d a)$ de $T$ é dada por

$$
F(t)=1-\exp \left(-t^{\alpha} e^{\lambda}\right)
$$

As funções de sobrevivência e risco de $T$ tem as seguintes expressões

$$
S(t)=\exp \left(-t^{\alpha} e^{\lambda}\right) \quad \text { e } \quad h(t)=\alpha e^{\lambda} t^{\alpha-1}
$$

respectivamente. A Figura 1.1 ilustrou que a função de risco de distribuição Weibull $h(t)$ é estritamente crescente para $\alpha>1$, estritamente decrescente para $\alpha<1$ e constante para $\alpha=1$; nesse caso $T$ segue uma distribuição exponencial com parâmetro $e^{\lambda}$, o que revela uma certa flexibilidade quanto ao comportamento da função de risco.
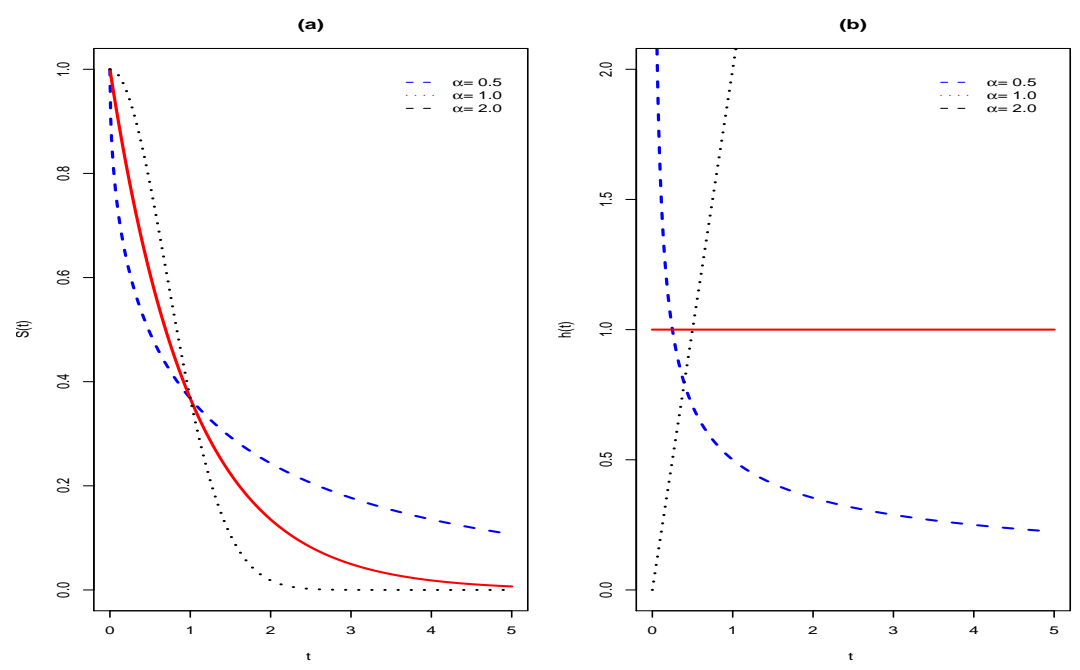

FIGURA 1.1: (a) Função de sobrevivência e (b) Função de risco da distribuição Weibull com $\lambda=0$ e diferentes valores de $\alpha$ selecionados

\subsubsection{Quantis e momentos}

Teorema 1.1. Seja T uma variável aleatória que tem distribuição Weibull com parâmetros $\alpha$ e $\lambda$. Então o k-ésimo momento de Té dado por:

$$
E\left[T^{k}\right]=\exp \left(-\frac{\lambda k}{\alpha}\right) \Gamma\left(\frac{k}{\alpha}+1\right)
$$

De acordo com o teorema 1.1, a média e a variância da distribuição Weibull são 
dadas por

$$
E[T]=\exp \left(-\frac{\lambda}{\alpha}\right) \Gamma\left(\frac{1}{\alpha}+1\right)
$$

e

$$
\operatorname{Var}[T]=\exp \left(-\frac{2 \lambda}{\alpha}\right)\left\{\Gamma\left(\frac{2}{\alpha}+1\right)-\left[\Gamma\left(\frac{1}{\alpha}+1\right)\right]^{2}\right\}
$$

sendo $\Gamma(z)=\int_{0}^{\infty} t^{z-1} e^{-t} \mathrm{dt}$ uma função gama.

O q-ésimo quantil da distribuição Weibull, obtido invertendo a função de distribuição acumulada de $T$, é dado por

$$
t_{q}=\left(-\log (1-q) / e^{\lambda}\right)^{1 / \alpha}
$$

e particularmente, a mediana é dada por

$$
t_{1 / 2}=\left(\log (2) / e^{\lambda}\right)^{1 / \alpha}
$$

\subsection{Critérios de comparação de modelos}

Na literatura, existe uma grande variedade de metodologias para comparar vários modelos, permitindo-se que seja selecionado o melhor para um determinado conjunto de dados; um dos critérios de comparação mais utilizado é o critério da ordenada preditiva condicional-CPO, apresentado por Gelfand et al. (1992) e Geisser \& Eddy (1979). Desse modo, temos em $\mathcal{D}$ os dados completos e em $\mathcal{D}^{(-i)}$ os dados com a i-ésima observação excluída; de onde denotamos a densidade a posteriori de $\boldsymbol{\vartheta}$ dada $\mathcal{D}^{(-i)}$ por $\pi\left(\boldsymbol{\vartheta} \mid \mathcal{D}^{(-i)}\right)$, $i=1, \ldots, n$. Para a i-ésima observação, o $C P O_{i}$ pode ser escrito como

$$
C P O_{i}=\int_{\vartheta} g\left(y_{i} \mid \boldsymbol{\vartheta}\right) \pi\left(\boldsymbol{\vartheta} \mid \mathcal{D}^{(-i)}\right) d \boldsymbol{\vartheta}=\left\{\int_{\vartheta} \frac{\pi(\boldsymbol{\vartheta} \mid \mathcal{D})}{g\left(y_{i} \mid \boldsymbol{\vartheta}\right)} d \boldsymbol{\vartheta}\right\}^{-1}
$$

em que $g\left(y_{i} \mid \boldsymbol{\vartheta}\right)$ é a função densidade de probabilidade. No contexto de análise de sobrevivência, $g\left(y_{i} \mid \boldsymbol{\vartheta}\right)=f\left(y_{i} ; \boldsymbol{\vartheta}\right)$ para os tempos observados do evento de interesse, e $g\left(y_{i} \mid \boldsymbol{\vartheta}\right)=$ $S\left(y_{i} ; \boldsymbol{\vartheta}\right)$ para os tempos censurados.

O maior valor de $C P O_{i}$ implica o melhor ajuste do modelo. Em geral, para os modelos complexos não há formas fechadas do $C P O_{i}$, porém, uma estimativa Monte Carlo 
para $C P O_{i}$, considerando uma amostra de tamanho $Q$ da distribuição a posteriori (Chen et al., 2000) é dada por

$$
\widehat{C P O}_{i}=\left\{\frac{1}{Q} \sum_{q=1}^{Q} \frac{1}{g\left(y_{i} \mid \boldsymbol{\vartheta}^{(q)}\right)}\right\}^{-1}
$$

Como em Cancho et al. (2010) utilizamos a estatística "log pseudo marginal likelihood-LPML definida por $L P M L=\sum_{i=1}^{n} \log \left(\widehat{C P O}_{i}\right)$. Assim, na seleção dos modelos, os maiores valores de LPML indicam o melhor modelo.

Outros critérios utilizados neste trabalho são o Deviance Information CriterionDIC (Spiegelhalter et al., 2002), o Expected Akaike Information Criterion-EAIC (Brooks, 2002), e o Expected Bayesian (or Schwarz) Information Criterion-EBIC (Carlin \& Louis, 2001), os quais são baseados na média a posteriori do desvio, que pode ser aproximada por $\bar{d}=\sum_{q=1}^{Q} d\left(\boldsymbol{\vartheta}_{q}\right) / Q$, no qual $d(\boldsymbol{\vartheta})=-2 \sum_{i=1}^{n} \log \left[g\left(y_{i} \mid \boldsymbol{\vartheta}\right)\right]$.

O critério DIC também pode ser estimado considerando amostras MCMC por $\widehat{D I C}=\bar{d}+\widehat{\rho_{d}}=2 \bar{d}-\widehat{d}$, em que $\rho_{d}=E\{d(\boldsymbol{\vartheta})\}-d\{E(\boldsymbol{\vartheta})\}$ é o número de parâmetros efetivos, no qual o desvio avaliado na média da posterior $d\{E(\boldsymbol{\vartheta})\}$ pode ser estimado como

$$
\widehat{d}=d\left(\frac{1}{Q} \sum_{q=1}^{Q} \boldsymbol{\vartheta}^{(q)}\right)
$$

Analogamente, os critérios EAIC e EBIC também podem ser aproximados por meio de $\widehat{E A I C}=\bar{d}+2 \#(\boldsymbol{\vartheta})$ e $\widehat{E B I C}=\bar{d}+\#(\boldsymbol{\vartheta}) \log (n)$, em que $\#(\boldsymbol{\vartheta})$ é o número dos parâmetros do modelo.

\subsection{Método de diagnóstico}

Após a modelagem é importante verificar as suposições feitas para o modelo, conduzindo para um estudo da robustez com o intuito de encontrar possíveis observações influentes, as quais podem causar distorções nos resultados da análise. Uma das propostas mais inovadoras em análise de sensibilidade foi apresentada por Cook (1986), que propôs avaliar a influência conjunta das observações sobre pequenas perturbações no modelo, ao invés da avaliação pela retirada individual ou conjunta de pontos. Essa metodologia é denominada influência local, e embora venha sendo aplicada com sucesso em diferentes 
áreas da estatística aplicada, observamos que dependendo da complexidade do modelo, a sua aplicação envolve extensas manipulações algébricas, e em alguns casos um duro trabalho computacional. Vários autores tem aplicado as técnicas de influência local, como Ortega et al. (2003), os quais apresentam uma aplicação a modelos de regressão log-gama generalizados com observações censuradas. Em Labra et al. (2005) é realizado um estudo de influência local em um modelo com erros de medição $t$ de Student com intercepto nulo, e em Ortega et al. (2006) é aplicado um estudo de influência local para o modelo de regressão logística.

Peng \& Dey (1995) apresentam duas distintas abordagens bayesianas para detectar observações influentes no ajuste de modelos de regressão, sendo uma baseada na distribuição a posteriori e a outra baseada na distribuição preditiva. Recentemente, Cho et al. (2009), propuseram um método bayesiano de análise de influência caso a caso para dados de sobrevivência, baseado na divergência Kullback-Leibler. Nesse trabalho, consideramos quatro medidas de divergência, propostas por Peng \& Dey (1995) para avaliar a influência de um caso nas distribuições a posteriori conjuntas e marginais fundamentadas. A seguir apresentamos essa metodologia.

\subsubsection{Análise de influência caso a caso}

Uma maneira comum de avaliar a influência de uma observação no ajuste de um modelo é por meio da deleção de casos (Cook \& Weisberg, 1982), em que o efeito de remover cada caso completamente da análise é estudado. Baseada no mesmo raciocínio, a metodologia da análise de influência caso a caso com um abordagem Bayesiana possibilita determinar quais casos podem influenciar nos resultados da análise.

Seja $D_{\psi}\left(P, P_{(-i)}\right)$, a qual denota a divergência $\psi$ entre $P$ e $P_{(-i)}$, em que $P$ é a distribuição a posteriori de $\boldsymbol{\vartheta}$ para dados completos, e $P_{(-i)}$ é a distribuição a posteriori de $\boldsymbol{\vartheta}$ sem i-ésimo caso. Especificamente,

$$
D_{\psi}\left(P, P_{(-i)}\right)=\int \psi\left(\frac{\pi\left(\boldsymbol{\vartheta} \mid \mathcal{D}^{(-i)}\right)}{\pi(\boldsymbol{\vartheta} \mid \mathcal{D})}\right) \pi(\boldsymbol{\vartheta} \mid \mathcal{D}) d \boldsymbol{\vartheta}
$$

$D_{\psi}\left(P, P_{(-i)}\right)$ mede o efeito de omitir o $i$-ésimo caso dos dados completos na distribuição $a$ posteriori de $\boldsymbol{\vartheta}$. Note que, em geral, $D_{\psi}\left(P, P_{(-i)}\right) \neq D_{\psi}\left(P_{(-i)}, P\right)$, e que $\psi$ é uma função 
convexa com $\psi(1)=0$; várias escolhas da função $\psi$ são dadas em Dey \& Birmiwal (1994). Por exemplo, se $\psi(z)=-\log (z)$ obtemos a divergência Kullback-Leibler $(K-L$ divergence); se $\psi(z)=(z-1) \log (z)$ temos que $J$-distance é uma versão simétrica da divergência Kullback-Leibler; se $\psi(z)=0.5|z-1|$ temos a distância variacional ou a norma $L_{1}$ e se $\psi(z)=(z-1)^{2}$, a divergência qui-quadrado $\left(\chi^{2}\right.$-divergence $)$ é obtida.

A relação entre o $C P O_{i}(1.4)$ e a medida da $\psi$-divergência é descrita na próxima proposição.

Proposição 1.1. A medida $\psi$-divergência pode ser escrita como

$$
D_{\psi}\left(P, P_{(-i)}\right)=E_{\vartheta \mid \mathcal{D}}\left[\psi\left(\frac{C P O_{i}}{g\left(y_{i} \mid \boldsymbol{\vartheta}\right)}\right)\right]
$$

em que o valor esperado é tomado com relação à distribuição conjunta a posteriori $\pi(\boldsymbol{\vartheta} \mid \mathcal{D})$.

Prova: Do teorema de Bayes a distribuição a posteriori do $\boldsymbol{\vartheta}$ é dada por

$$
\pi(\boldsymbol{\vartheta} \mid \mathcal{D})=\frac{\pi(\boldsymbol{\vartheta}) \prod_{j \in \mathcal{D}} g\left(y_{j} \mid \boldsymbol{\vartheta}\right)}{\int_{\boldsymbol{\vartheta} \in \Theta} \pi(\boldsymbol{\vartheta}) \prod_{j \in \mathcal{D}} g\left(y_{j} \mid \boldsymbol{\vartheta}\right) d \boldsymbol{\vartheta}}
$$

em que $\pi(\boldsymbol{\vartheta})$ e $\prod_{i=1}^{n} g\left(y_{i} \mid \boldsymbol{\vartheta}\right)$ representam a distribuição a priori a e a função de verossimilhança de $\boldsymbol{\vartheta}$, respectivamente. A razão entre as distribuições a posteriori é dada por

$$
\begin{aligned}
\frac{\pi\left(\boldsymbol{\vartheta} \mid \mathcal{D}^{(-i)}\right)}{\pi(\boldsymbol{\vartheta} \mid \mathcal{D})} & =\frac{\pi(\boldsymbol{\vartheta}) \prod_{j \in D^{(i)}} g\left(y_{i} \mid \boldsymbol{\vartheta}\right)}{\int_{\boldsymbol{\vartheta} \in \Theta} \pi(\boldsymbol{\vartheta}) \prod_{j \in D^{(i)}} g\left(y_{j} \mid \boldsymbol{\vartheta}\right) d \boldsymbol{\vartheta}} \times \frac{\int_{\boldsymbol{\vartheta} \in \Theta} \pi(\boldsymbol{\vartheta}) \prod_{j \in D} g\left(y_{j} \mid \boldsymbol{\vartheta}\right) d \boldsymbol{\vartheta}}{\pi(\boldsymbol{\vartheta}) \prod_{j \in D} g\left(y_{j} \mid \boldsymbol{\vartheta}\right)} \\
& =\frac{1}{g\left(y_{i} \mid \boldsymbol{\vartheta}\right)} \times \frac{\int_{\boldsymbol{\vartheta} \in \Theta} \pi(\boldsymbol{\vartheta}) \prod_{j \in D} g\left(y_{j} \mid \boldsymbol{\vartheta}\right) d \boldsymbol{\vartheta}}{\int_{\boldsymbol{\vartheta} \in \Theta} \frac{1}{g\left(y_{i} \mid \boldsymbol{\vartheta}\right)} \pi(\boldsymbol{\vartheta}) \prod_{j \in D} g\left(y_{j} \mid \boldsymbol{\vartheta}\right) d \boldsymbol{\vartheta}} \\
& =\frac{\left(\int_{\boldsymbol{\vartheta} \in \Theta} \frac{1}{g\left(y_{i} \mid \boldsymbol{\vartheta}\right)} \pi(\boldsymbol{\vartheta} \mid \mathcal{D}) d \boldsymbol{\vartheta}\right)^{-1}}{g\left(y_{i} \mid \boldsymbol{\vartheta}\right)}=\frac{C P O_{i}}{g\left(y_{i} \mid \boldsymbol{\vartheta}\right)}
\end{aligned}
$$

Então, (1.6) pode ser obtido, completando a prova.

De acordo com a proposição 1.1, a $K$-L divergence pode ser expressa como uma esperança da posteriori, dada por

$$
\begin{aligned}
D_{\mathrm{K}-\mathrm{L}}\left(P, P_{(-i)}\right) & =-E_{\boldsymbol{\vartheta} \mid \mathcal{D}}\left\{\log \left(C P O_{i}\right)\right\}+E_{\boldsymbol{\vartheta} \mid \mathcal{D}}\left\{\log \left[g\left(y_{i} \mid \boldsymbol{\vartheta}\right)\right]\right\} \\
& =-\log \left(C P O_{i}\right)+E_{\boldsymbol{\vartheta} \mid \mathcal{D}}\left\{\log \left[g\left(y_{i} \mid \boldsymbol{\vartheta}\right)\right]\right\}
\end{aligned}
$$

De (1.6) podemos calcular $D_{\psi}\left(P, P_{(-i)}\right)$ por amostragem a partir da distribuição $a$ 
posteriori do $\boldsymbol{\vartheta}$ através de métodos MCMC. Seja $\boldsymbol{\vartheta}^{(1)}, \ldots, \boldsymbol{\vartheta}^{(Q)}$ uma amostra de tamanho $Q$ de $\pi(\boldsymbol{\vartheta} \mid \mathcal{D})$. Então, a estimativa Monte Carlo $D_{\psi}\left(P, P_{(-i)}\right)$ é dada por

$$
\widehat{D_{\psi}}\left(P, P_{(-i)}\right)=\frac{1}{Q} \sum_{q=1}^{Q} \psi\left(\frac{\widehat{C P O}_{i}}{g\left(y_{i} \mid \boldsymbol{\vartheta}^{(q))}\right.}\right)
$$

De (1.8) a estimativa Monte Carlo da $K$ - $L$ divergence é dada por

$$
\widehat{D_{\mathrm{K}-\mathrm{L}}}\left(P, P_{(-i)}\right)=-\log \left(\widehat{C P O}_{i}\right)+\frac{1}{Q} \sum_{q=1}^{Q} \log \left[g\left(y_{i} \mid \boldsymbol{\vartheta}^{(q)}\right)\right]
$$

Foi ressaltado por Peng \& Dey (1995) e Weiss (1996) que pode ser difícil detectar o ponto de corte da medida de divergência de modo a determinar se um pequeno subconjunto de observações é influente ou não, por isso, neste contexto usaremos esta proposta dada. Assim, considere uma moeda enviesada com probabilidade de sucesso $p$, então a medida de $\psi$-divergência entre as moedas não enviesada e enviesada é dada por

$$
D_{\psi}\left(f_{0}, f_{1}\right)=\int \psi\left(\frac{f_{0}(x)}{f_{1}(x)}\right) f_{1}(x) d x
$$

em que $f_{0}(x)=p^{x}(1-p)^{1-x}$ e $f_{1}(x)=0,5, x=0 ; 1$. No entanto, se $D_{\psi}\left(f_{0}, f_{1}\right)=d_{\psi}(p)$, então pode ser facilmente verificado que $d_{\psi}$ satisfaz a seguinte equação

$$
d_{\psi}(p)=\frac{\psi(2 p)-\psi(2(1-p))}{2}
$$

Note que a função $d_{\psi}(\cdot)$ é simétrica em torno de $p=0,5$ e atinge o valor mínimo neste ponto, $d_{\psi}(0,5)=0$ e $f_{0}=f_{1}$. Além disso, se $p>0,75$ (ou $p \leq 0,25$ ), como uma moeda fortemente enviesada, temos que $d_{\mathrm{K}-\mathrm{L}}(0,75)=0,14 ; d_{\mathrm{J}}(0,75)=0,27$ e $d_{L_{1}}(0,75)=$ $d_{\chi^{2}}(0,75)=0,25$. Assim, para as medidas de $K$-L divergence, J-distance, $L_{1}$-distance e $\chi^{2}$-divergence, consideramos uma observação influente quando $d_{\mathrm{K}-\mathrm{L}}>0,14 ; d_{\mathrm{J}}>0,27$; $d_{L_{1}}>0,25$ ou $d_{\chi^{2}}>0,25$.

Neste trabalho propomos uma classe de modelos de sobrevivência sem e com fração de cura, sob os diferentes esquemas de ativação latentes, com o objetivo de estudar as propriedades matemáticas padrões dos modelos e mostrar a sua flexibilidade por meio dos estudos de simulações e das aplicações aos vários conjuntos de dados reais. Os métodos 
de máxima verossimilhança e MCMC foram utilizados para desenvolver procedimentos de inferência clássica e Bayesiana, respectivamente. A implementação computacional do trabalho foi desenvolvida no software R (R Development Core Team, 2010). Além disso, os métodos de análise de influência caso a caso apresentados na Seção 1.4 foram utilizados para investigar os possíveis problemas com o modelo ajustado.

\subsection{Organização dos capítulos}

No Capítulo 2 apresentamos a distribuição Weibull-Binomial-Negativa (WBN) em um cenário de causas competitivas, assumindo que o número de causas é modelado pela distribuição Binomial Negativa (Piegorsch, 1990) truncada em zero e que essas causas competitivas tem diferentes mecanismos de ativação. Determinamos algumas propriedades padrão desta nova distribuição e os aspectos de inferência através do método de máxima verossimilhança foram discutidos. Finalmente, a aplicação de dois conjuntos de dados reais mostrou a flexibilidade da nova distribuição.

No Capítulo 3 propomos o modelo de regressão locação escala usando a distribuição WBN e o denominamos o modelo de regressão Log-WBN (LWBN), considerando a mesma estrutura de causas competitivas do Capítulo 2. Desenvolvemos métodos Bayesianos via Monte Carlo em cadeias de Markov (MCMC) para este modelo de regressão e realizamos um estudo de diagnóstico.

Por fim, no Capítulo 4, propomos um modelo de sobrevivência de longa duração para dados de sobrevivência com fração de cura, considerando que o número de causas (ou riscos) latentes tem distribuição Binomial Negativa e que essas causas são ativadas por meio de diferentes mecanismos de ativação. Desenvolvemos os procedimentos de inferência em uma perspectiva clássica e Bayesiana. No final deste capítulo, ajustamos o modelo de sobrevivência com fração de cura para um conjunto de dados de sobrevivência, e realizamos uma análise de sensibilidade na estimativa dos parâmetros a posteriori do melhor ajuste aos dados. Algumas perspectivas de trabalhos futuros foram enumeradas no Capítulo 5. 


\section{Capítulo 2}

\section{Modelo Weibull-Binomial-Negativo}

\subsection{Introdução}

A distribuição de Weibull é uma das distribuições mais utilizadas para modelar os dados de tempo de vida, sendo usada para modelar dados de tempo de vida com função de risco monótona ou constante, mas não é adequada para modelar situações em que a função de risco tem forma de banheira ou unimodal, as quais são comuns no estudo de análise de sobrevivência. Com a finalidade de modelar esses tipo de riscos, várias distribuições tem sido propostas na literatura para estender a distribuição de Weibull, como a distribuição Weibull-Exponenciada (WE) introduzida por Mudholkar \& Srivastava (1993), que apresenta função de risco unimodal, e a distribuição Weibull estendida proposta por Xie et al. (2002) e a distribuição Weibull modificada (WM), proposta por Lai et al. (2003), que tem função de risco em forma de banheira. Além disso, Silva et al. (2010) estudaram a distribuição Beta-Weibull modificada que generaliza a distribuição WM, WE e a distribuição Beta-Weibull introduzida por Famoye et al. (2005), cuja função de risco tem formatos monótono, unimodal e banheira.

Recentemente Barreto-Souza et al. (2011) propuseram a distribuição WeibullGeométrica (WG) obtida a partir de uma composição das distribuições Weibull e Geométrica, em um cenário de causas (ou riscos) competitivas (Basu \& Klein, 1982), em que somente o mínimo tempo de vida é observado, isto é, somente uma única causa ativada. Tal distribuição generaliza a distribuição Geométrica Exponencial Estendida (GEE) proposta 
por Adamidis et al. (2005) e a distribuição Geométrica Exponencial (GE) introduzida por Adamidis \& Loukas (1998). A função de risco da distribuição WG tem formas mais gerais e é útil para modelar os dados com taxa de falha unimodal.

Neste capítulo, propomos a distribuição Weibull-Binomial-Negativa (WBN), assumindo uma estrutura de ativação latente para explicar a ocorrência de um evento de interesse, em que o número de causas competitivas é modelado pela distribuição Binomial Negativa, as quais podem ter diferentes mecanismos de ativação, e os tempos não observados devido aos riscos seguem a distribuição Weibull. A distribuição proposta apresenta como caso particular os modelos propostos por Adamidis \& Loukas (1998), Kus (2007), Barreto-Souza et al. (2011), Roman et al. (2011), G. et al. (2011). Além disso, a distribuição WBN é flexível porque suas funções de risco tem formas não monótonas.

Em seguida, na Seção 2.2, formulamos o modelo proposto (WBN) baseado na distribuição Binomial Negativa, na Seção 2.3 apresentamos o procedimento inferencial baseado no método da verossimilhança assumindo que tempos não observados devido aos riscos seguem a distribuição Weibull. Um estudo de simulação foi realizado com objetivo de comparar os modelos propostos WBN na presença dos diferentes esquemas de ativação é apresentado na Seção 2.4. Uma aplicação com dois conjuntos de dados reais é apresentada na Seção 2.5 e finalmente, na Seção 2.6, é apresentada a conclusão deste Capítulo.

\subsection{Formulação do modelo}

Suponha que para um indivíduo da população, $M$ seja o número de causas (ou riscos) de ocorrência de um particular evento de interesse; desse modo, assumimos que $M$ segue uma distribuição Binomial Negativa com parâmetros $\eta$ e $\theta$ (Piegorsch, 1990), com a seguinte função de probabilidade

$$
P(M=m)=p_{m}=\frac{\Gamma\left(\eta^{-1}+m\right)}{\Gamma\left(\eta^{-1}\right) m !}\left(\frac{\theta \eta}{1+\theta \eta}\right)^{m}\left(\frac{1}{1+\eta \theta}\right)^{1 / \eta}
$$

$m=0,1,2, \ldots, \theta>0$ e $\eta>-1 / \theta$, de modo que

$$
E[M]=\theta, \quad \operatorname{Var}(M)=\theta+\eta \theta^{2}
$$


Como observado por Rodrigues et al. (2009) a variância de $M$ em (2.2) proporciona, simultaneamente, dois cenários importantes: o de sobredispersão, que ocorre quando $\eta>$ 0 e pode ser interpretado no caso de pesquisas biológicas na área de câncer, como um agrupamento de células cancerígenas ou tumorais, e a subdispersão, que ocorre no sentido inverso quando $\eta$ assume valores negativos. A distribuição de probabilidade em (2.1) é muito flexível no sentido de fornecer ligações entre as distribuições Geométrica, Poisson e Binomial Negativa, pois se $\eta=1$ temos o modelo geométrico e se $\eta \rightarrow 0$ temos o modelo de Poisson.

Quando $M=0$ não há causas que fazem com que o evento de interesse ocorra, desse modo o tempo para que o evento ocorra pode ser tomado como infinito, esta situação é de interesse nos modelos de sobrevivência de longa duração. No entanto, neste capítulo nossa atenção é restrita aos tempos de ocorrência finita, então presume-se que há pelo menos uma causa. Portanto, assumimos que o número de causas $M$ da ocorrência de um particular interesse tem distribuição BN truncada em zero com a função de probabilidade dada por

$$
P(M=m)=p_{m}=\frac{\Gamma\left(\eta^{-1}+m\right)}{\Gamma\left(\eta^{-1}\right) m !}\left(\frac{\eta \theta}{1+\eta \theta}\right)^{m}(1+\eta \theta)^{-1 / \eta}\left(1-(1+\eta \theta)^{-1 / \eta}\right)^{-1}
$$

$\operatorname{com} E[M]=\frac{\theta}{1-(1+\eta \theta)^{-1 / \eta}}$ e $\operatorname{Var}[M]=\frac{\eta \theta^{2}+\theta-\left(\eta \theta^{2}+\theta^{2}+\theta\right)(1+\eta \theta)^{-1 / \eta}}{\left(1-(1+\eta \theta)^{-1 / \eta}\right)^{2}}$.

Dado $M=m$ e sejam $T_{i}(i=1,2, \cdots, M)$ variáveis aleatórias não negativas e contínuas independentes entre si com função de distribuição $G(\cdot)$ e independentes de $M$, representando o tempo de ocorrência do evento de interesse devido à i-ésima causa (ou risco). Assim, o tempo de ocorrência do evento de interesse a ser observado pode ser definido pela variável aleatória $Y=T_{(R)}, R=1, \cdots, M$, em que $T_{(1)} \leq \cdots \leq T_{(M)}$ são as estatísticas de ordem de $T_{j}$, e $R$ dependente de $M$, sendo que $R$ pode ser uma constante fixa que depende de $M$, ou pode ser uma variável aleatória com distribuição especificada através de uma distribuição condicional de $R$ dado $M \geq 1$. Neste trabalho consideramos três casos específicos para $R$.

Considerando as condições acima, podemos demonstrar que a função de distribui- 
ção condicional de $Y$ dado $M$ e $R$ tem expressão

$$
F_{Y \mid m, r}(y)=P[Y \leq y \mid M=m, R=r]=\sum_{j=r}^{m}\left(\begin{array}{c}
m \\
j
\end{array}\right) G(y)^{j}(1-G(y))^{m-j}
$$

Assumindo que $R$ é uma variável aleatória com distribuição condicional de $R$ dado $M \geq 1$, dado por uma distribuição uniforme discreta em $1,2, \cdots, M$ (ativação aleatória) com probabilidade $1 / M$, a distribuição marginal de $Y$ é dada por

$$
\begin{aligned}
F(y) & =\sum_{m=1}^{\infty} \sum_{r=1}^{m} P\left[T_{(R) \leq y \mid M=m, R=r}\right] P[R=r \mid M=m] P[M=m] \\
& =1-\sum_{m=1}^{\infty}\left\{\sum_{r=0}^{m}(m-1) B(r, m, G(y))\right\} \frac{1}{m} P[M=m] \\
& =1-(1-G(y)) \sum_{m=1}^{\infty} p_{m}=G(y),
\end{aligned}
$$

em que $B(x, m, G(y))$ é a $f d p$ da distribuição binomial com parâmetros $m$ e $G(y)$, e $P[M=$ $m]$ é dada em (2.3). Nesse caso, a função distribuição marginal de $Y$ em (2.5) é a mesma da distribuição das variáveis aleatórias $T_{j}$ 's.

Considerando $R=r$ (fixo), então a distribuição marginal de $Y$ é dada por

$$
F(y)=\sum_{m=1}^{\infty} I B(G(y) ; r, m-r+1) p_{m}=\sum_{m=1}^{\infty}\left(\begin{array}{c}
m-1 \\
r-1
\end{array}\right) m p_{m} \int_{0}^{G(y)} u^{r-1}(1-u)^{m-r} d u
$$

em que $I B(x ; a, b)$ é uma função beta incompleta e $p_{m}=P[M=m]$ é dada em $(2.3)$.

Se $R=1$, isto implica que o evento de interesse ocorre devido à ativação de qualquer uma destas causas (fatores latentes), isto é, $Y=T_{(1)}=\min \left\{T_{1}, \cdots, T_{M}\right\}$. Neste caso, conhecido como esquema de primeira ativação (Cooner et al., 2007), a distribuição marginal de $Y$ em (2.6) é dada por

$$
F(y)=\frac{1-(1+\eta \theta G(y))^{-1 / \eta}}{1-(1+\eta \theta)^{-1 / \eta}}, y>0,
$$

e a função de densidade correspondente é dada por

$$
f(y)=\frac{\theta g(y)(1+\theta \eta G(y))^{-(1 / \eta+1)}}{1-(1+\theta \eta)^{-1 / \eta}} .
$$

Considerando (2.7) e (2.8), a função de sobrevivência e a função de taxa de falha do modelo 
com primeira ativação são dadas, respectivamente, por

$$
S(y)=\frac{(1+\theta \eta G(y))^{-1 / \eta}-(1+\theta \eta)^{-1 / \eta}}{1-(1+\theta \eta)^{-1 / \eta}}
$$

e

$$
h(y)=\frac{\theta g(y)(1+\theta \eta G(y))^{-(1 / \eta+1)}}{(1+\theta \eta G(y))^{-1 / \eta}-(1+\theta \eta)^{-1 / \eta}} .
$$

Assumindo que o evento de interesse acontece depois que todas as $M$ causas tenham ocorrido, então, $R=M$, e $Y=T_{(M)}=\max \left\{T_{1}, \cdots, T_{M}\right\}$, a qual é conhecida como esquema de última ativação (Cooner et al., 2007). A distribuição marginal de $Y$ em (2.6) é dada por

$$
F(y)=\frac{(1+\eta \theta(1-G(y)))^{-1 / \eta}-(1+\eta \theta)^{-1 / \eta}}{1-(1+\eta \theta)^{-1 / \eta}}, y>0
$$

e a função de densidade correspondente é dada por

$$
f(y)=\frac{\theta f(y)(1+\theta \eta(1-G(y)))^{-(1 / \eta+1)}}{1-(1+\theta \eta)^{-1 / \eta}}
$$

Considerando (2.11) e (2.12), a função de sobrevivência e a função de taxa de falha do modelo com primeira ativação são dadas, respectivamente, por

$$
S(y)=\frac{1-(1+\theta \eta(1-G(y)))^{-1 / \eta}}{1-(1+\theta \eta)^{-1 / \eta}},
$$

e

$$
h(y)=\frac{\theta f(y)(1+\theta \eta(1-G(y)))^{-(1 / \eta+1)}}{1-(1+\theta \eta(1-G(y)))^{-1 / \eta}}
$$

A seguinte proposição mostra a relação entre as funções distribuições dadas por $(2.5),(2.7)$ e $(2.11)$, denotadas, respectivamente, por $F_{\text {aleatória }}(y), F_{\text {primeira }}(y)$ e $F_{\text {última }}(y)$.

Proposição 2.1. Sob condições dos modelos em (2.5), (2.7) e (2.11) para qualquer função de distribuição $G(y)$, temos que $F_{\text {última }}(y) \leq F_{\text {aleatória }}(y) \leq F_{\text {primeira }}(y), \forall y>0$.

Prova. Sabemos que $\frac{1-(1+\eta \theta G(y))^{-1 / \eta}}{G(y)}$ é uma função crescente em relação de $y$, assim podemos obter o valor mínimo quando $y=0$, isto é, $\lim _{y \rightarrow 0} \frac{1-(1+\eta \theta G(y))^{-1 / \eta}}{G(y)}=1-(1+$ $\theta \eta)^{-1 / \eta}$, implica que $\frac{1-(1+\eta \theta G(y))^{-1 / \eta}}{G(y)} \geq 1-(1+\theta \eta)^{-1 / \eta}, \forall y$. Logo, $G(y) \leq \frac{1-(1+\eta \theta G(y))^{-1 / \eta}}{1-(1+\eta \theta)^{-1 / \eta}}, \mathrm{o}$ que implica que $F_{\text {primeira }}(y) \geq F_{\text {aleatória }}(y), \forall y>0$. Analogamente para provar $F_{\text {última }}(y) \leq$ $F_{\text {aleatória }}(y)$. 
Considerando diferentes escolhas para a distribuição de variáveis aleatórias $T_{j}$ 's, novas famílias de distribuições podem ser obtidas. Neste trabalho, consideramos que as variáveis aleatórias $T_{j}$ 's seguem a distribuição Weibull com parâmetros $\alpha$ e $\lambda$ dada em (1.1). Assim, de (2.5), (2.7) e (2.11) obtemos a distribuição sob ativação aleatória (WBN-AA), a distribuição WBN sob primeira ativação (WBN-PA) e a distribuição WBN sob última ativação (WBN-UA), respectivamente. Note que neste caso, a distribuição WBN-AA é a mesma distribuição Weibull dada em (1.1). As principais características das distribuições WBN-PA e WBN-UA são apresentadas a seguir.

A Figura 2.1 mostra os comportamentos distintos das funções de sobrevivência e risco dos modelos sob esquemas de primeira ativação, última ativação e ativação aleatória, ilustrando a flexibilidade do modelo proposto.

(a)

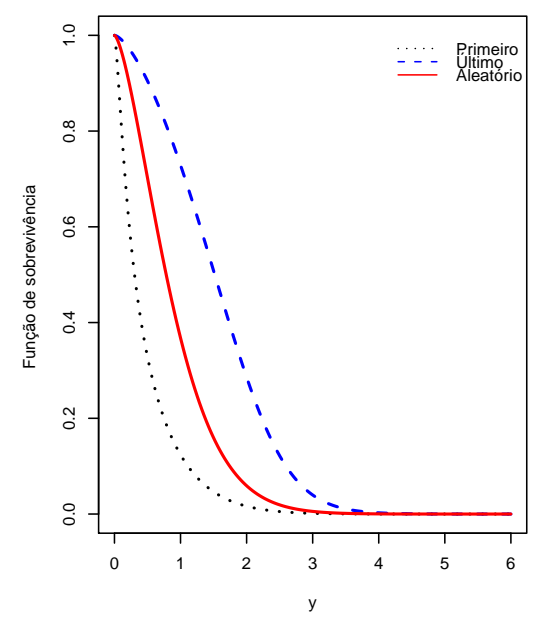

(b)

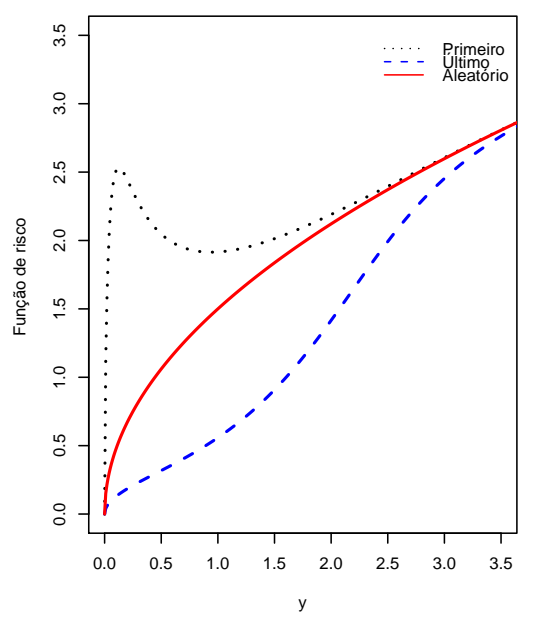

FIGURA 2.1: (a)Função de sobrevivência; (b)Função de risco da distribuição WBN com parâmetros $\lambda=0, \alpha=1,5, \eta=10$ e $\theta=2$.

\subsubsection{A distribuição WBN sob esquema de primeira ativação}

Seja Y uma variável aleatória não negativa que segue a distribuição WBN-PA com o vetor de parâmetros $\boldsymbol{\xi}=(\alpha, \lambda, \eta, \theta)$, a $f d p$ de $Y$ é dada por

$$
f(y ; \xi)=\frac{\theta \alpha y^{\alpha-1} \exp \left(\lambda-y^{\alpha} e^{\lambda}\right)\left[1+\eta \theta\left(1-\exp \left(-y^{\alpha} e^{\lambda}\right)\right)\right]^{-(1 / \eta+1)}}{1-(1+\eta \theta)^{-1 / \eta}}, \quad y>0 .
$$

Alguns sub-modelos da distribuição WBN-PA (2.15) são obtidos a seguir. Se 
$\eta=1$ temos a distribuição WG-PA, introduzida por Barreto-Souza et al. (2011); se $\eta \rightarrow 0$, obtemos a distribuição Poisson Weibull sob primeira ativação (WP-PA). Fixando $\alpha=1$, obtemos a distribuição Exponencial-Binomial-Negativa (EBN-PA), e quando $\eta=1$ temos a distribuição Exponencial-Geométrica (EG-PA) (Adamidis \& Loukas, 1998); quando $\eta \rightarrow 0$, obtemos a distribuição Exponencial-Poisson (EP-PA) (Kus, 2007). Além disso, quando $\theta \rightarrow 0$ a distribuição WBN-PA (2.15) leva à distribuição de Weibull (1.1). As funções de densidade da distribuição WBN-PA são exibidas na Figura 2.2 para $\lambda=0$ fixo e alguns valores do vetor $\phi=(\alpha, \theta)$ and $\eta=-1,-0,5 ; 0,01 ; 1,0$ e 10 .
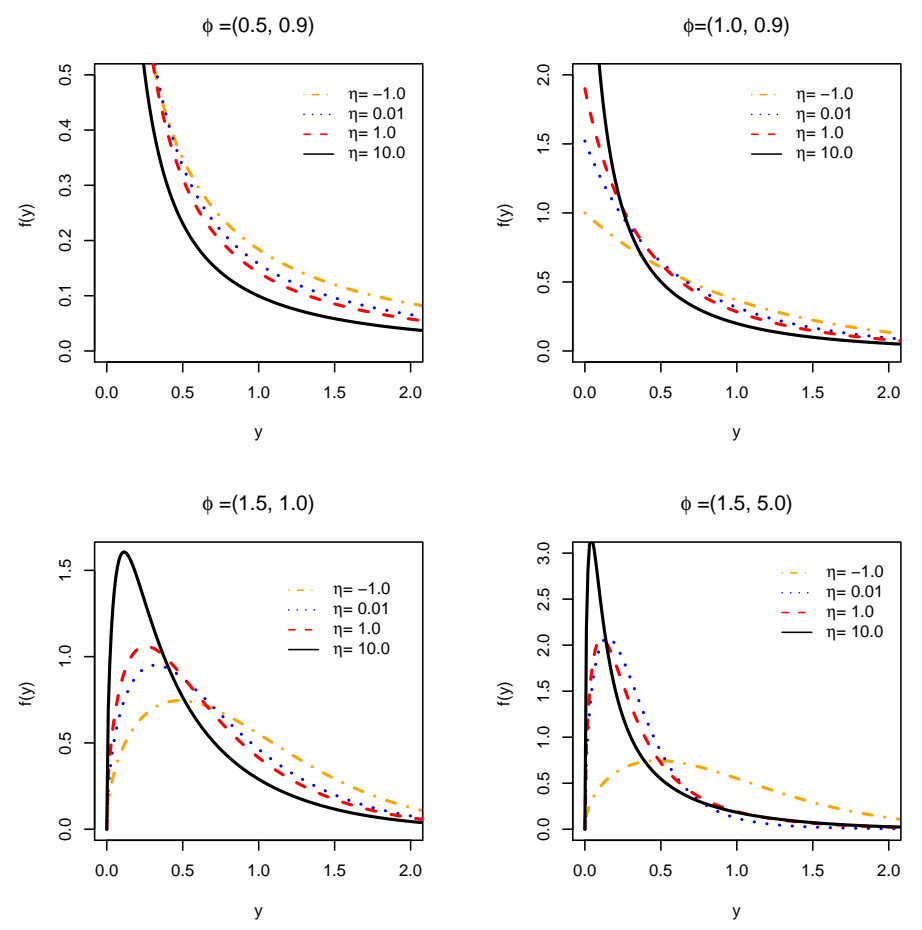

FIGURA 2.2: Função densidade de probabilidade da distribuição WBN-PA para alguns valores selecionados

Note que a $f d p$ da distribuição WBN-PA dada em (2.15) também pode ser escrita como

$$
f(y ; \xi)=\sum_{m=1}^{\infty} f(y \mid m, \alpha, \lambda) p_{m},
$$

em que $f(y \mid m, \alpha, \lambda)=m \alpha y^{\alpha-1} \exp \left(\lambda-m e^{\lambda} y^{\alpha}\right)$ é uma distribuição Weibull com parâmetro escalar $\lambda+\log (m)$, e o parâmetro de forma $\alpha$, e $p_{m}$ é dado em (2.3). Então, a $f d a$ da WBN-PA tem a seguinte expressão

$$
F(y ; \xi)=\sum_{m=1}^{\infty} F(y \mid m, \alpha, \lambda) p_{m}
$$


em que $F=(y \mid m, \alpha, \lambda)$ denota a $f d a$ da distribuição Weibull acumulada com o parâmetro de escala $\lambda+\log (m)$ e o parâmetro de forma $\alpha$. Portanto, a distribuição WBN-PA é uma mistura infinita das distribuições de Weibull, esse resultado permite a obtenção de algumas propriedades matemáticas desta nova distribuição.

A $f d a$ da distribuição WBN-PA é dada por

$$
F(y ; \xi)=\frac{1-\left(1+\eta \theta\left(1-\exp \left(-y^{\alpha} e^{\lambda}\right)\right)\right)^{-1 / \eta}}{1-(1+\eta \theta)^{-1 / \eta}}, \quad y>0
$$

As funções de sobrevivência e de risco da distribuição WBN-PA são dadas por

$$
S(y ; \xi)=\frac{\left(1+\eta \theta\left(1-\exp \left(-y^{\alpha} e^{\lambda}\right)\right)\right)^{-1 / \eta}-(1+\eta \theta)^{-1 / \eta}}{1-(1+\eta \theta)^{-1 / \eta}}, \quad y>0
$$

e

$$
h(y ; \xi)=\frac{\theta \alpha y^{\alpha-1} \exp \left(\lambda-y^{\alpha} e^{\lambda}\right)\left[1+\eta \theta\left(1-\exp \left(-y^{\alpha} e^{\lambda}\right)\right)\right]^{-(1 / \eta+1)}}{\left(1+\eta \theta\left(1-\exp \left(-y^{\alpha} e^{\lambda}\right)\right)\right)^{-1 / \eta}-(1+\eta \theta)^{-1 / \eta}}, \quad y>0,
$$

respectivamente.

A função de risco (2.20) é decrescente para $0<\theta \leq 1$ e crescente para $\theta>1$. No entanto, para outros valores de parâmetros, ele pode assumir diferentes formas e as funções de risco convergem para a mesma forma quando $y$ cresce. A Figura 2.3 ilustra algumas das formas possíveis da função de risco para valores selecionados do vetor $\phi=(\alpha, \theta)$ e $\eta=-1,0 ;-0,5 ; 0,01 ; 1,0$ e 10,0. As Figuras mostram que a função de risco da nova distribuição é muito mais flexível do que da distribuição WG. 

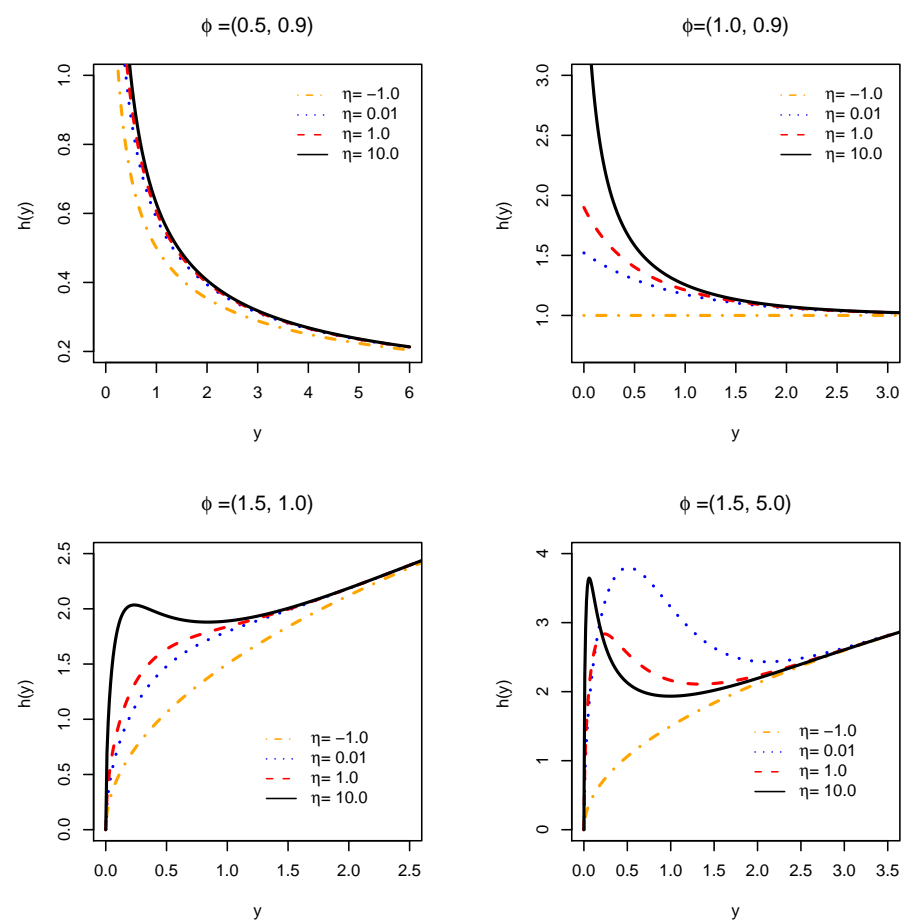

FIGURA 2.3: Função de risco da distribuição WBN-PA para alguns valores selecionados

\section{Quantis e momentos}

O quantil $q$ da distribuição WBN-PA é obtido invertendo-se a função densidade acumulada da distribuição WBN-PA e é dado por

$$
y_{q}=G^{-1}\left(\frac{\left[1-q\left(1-p_{0}\right)\right]^{-\eta}-1}{\eta \theta}\right)
$$

Em particular, a mediana é dada por $y_{1 / 2}=G^{-1}\left(\frac{\left[1-\frac{1}{2}\left(1-p_{0}\right)\right]^{-\eta}-1}{\eta \theta}\right)$, na qual $G^{-1}(y)=$ $\left(-\log (1-y) / e^{\lambda}\right)^{1 / \alpha}$.

O k-ésimo momento ordinário de $Y$ é dado por

$$
\begin{aligned}
E\left[Y^{k}\right] & =e^{-\lambda k / \alpha} \Gamma\left(\frac{k}{\alpha}+1\right) \sum_{m=1}^{\infty} m^{-k / \alpha} p_{m} \\
& =\frac{e^{-\lambda k / \alpha} \Gamma\left(\frac{k}{\alpha}+1\right)}{(1+\eta \theta)^{1 / \eta}-1} \varphi(k, \alpha, \eta, \theta)
\end{aligned}
$$

em que $\varphi(k, \alpha, \eta, \theta)=\sum_{m=1}^{\infty} m^{-k / \alpha} \frac{\Gamma\left(\eta^{-1}+m\right)}{\eta^{-1} m !}\left(\frac{\eta \theta}{1+\eta \theta}\right)^{m}<\infty$, que pode ser provado pelo critério de razão. 
Em um caso especial, quando $k / \alpha$ é um número inteiro positivo, para $\alpha>0$ e $k$ assumindo os valores inteiros positivos, podemos reescrever o k-ésimo momento ordinário de $Y$ como

$$
E\left[Y^{k}\right]=\frac{\theta e^{-\lambda k / \alpha} \Gamma \frac{k}{\alpha}+1}{(1+\eta \theta)\left[(1+\eta \theta)^{1 / \eta}-1\right]} F_{\frac{k}{\alpha}+2, \frac{1}{\alpha}+1}\left(\left[1, \cdots, 1, \frac{1+\eta}{\eta}\right],[2, \cdots, 2], \frac{\eta \theta}{1+\eta \theta}\right) .
$$

em que $F_{p, q}(\mathbf{n}, \mathbf{d}, \lambda)$ é a função hipergeométrica generalizada, também conhecida como função hipergeométrica estendida de Barnes, que é definida por

$$
F_{p, q}(\mathbf{n}, \mathbf{d}, \lambda)=\sum_{k=0}^{\infty} \frac{\lambda^{k} \prod_{i=1}^{p} \Gamma\left(n_{i}+k\right) \Gamma^{-1}\left(n_{i}\right)}{\Gamma(k+1) \prod_{i=1}^{q} \Gamma\left(d_{i}+k\right) \Gamma^{-1}\left(d_{i}\right)}
$$

no qual $\mathbf{n}=\left[n_{1}, \cdots, n_{p}\right], p$ é o número de operados de $\mathbf{n}, \mathbf{d}=\left[d_{1}, \cdots, d_{q}\right]$ e $q$ é o número de operados de $\mathbf{d}$. A função hipergeométrica generalizada é rápida e prontamente disponível em vários softwares matemáticos, como Maple. Além disso o valor da serie $\varphi(k, \alpha, \eta, \theta)$ também pode ser obtido numericamente.

Várias expressões de forma fechada podem ser obtidas a partir de (2.21) como casos particulares. O momento central $\mu_{p}$ e o cumulante $\left(\kappa_{s}\right)$ do $Y$ são facilmente obtidos a partir dos momentos ordinários dados por

$$
\mu_{p}=\sum_{r=0}^{p}\left(\begin{array}{l}
p \\
r
\end{array}\right) \mu_{1}^{\prime p} \mu_{p-r}^{\prime} \quad \text { e } \quad \kappa_{p}=\mu_{p}^{\prime}-\sum_{r=1}^{p-1}\left(\begin{array}{c}
p-1 \\
r-1
\end{array}\right) \kappa_{r} \mu_{p-r}^{\prime}
$$

respectivamente. As medidas de assimetria e curtose podem ser obtidas a partir das relações clássicas: Assimetria $(Y)=\kappa_{3} / \kappa^{3 / 2}$ e $\operatorname{Curtose}(Y)=\kappa_{4} / \kappa_{2}^{2}$. A Tabela 2.1 apresenta o momento ordinário, variância, assimetria e curtose da distribuição WBN-PA com $\lambda=0$; $\alpha=0,5 ; 1,0 ; 1,5$ e $\theta=0,9$.

\section{Função geradora de momentos}

A função geradora de momentos $(f g m) M(t)=E[\exp (t Y)]$, obtida através da expansão em série de potências para a função exponencial e também por meio de (2.16), é escrita como

$$
M(t)=\sum_{m=1}^{\infty} p_{m} \sum_{n=0}^{\infty} \frac{t^{n}\left(e^{\lambda} m\right)^{-n / \alpha}}{n !} \Gamma\left(1+\frac{n}{2}\right)
$$


TABELA 2.1: Momento ordinário, variância, assimetria e curtose da distribuição WBN-PA para alguns valores selecionados

\begin{tabular}{clccccc}
\hline$\alpha$ & & $\eta=0,01$ & $\eta=1$ & $\eta=2$ & $\eta=5$ & $\eta=10$ \\
\hline 0,5 & $k=1$ & 0,823 & 0,575 & 0,447 & 0,274 & 0,170 \\
& $k=2$ & 9,001 & 6,180 & 4,767 & 2,892 & 1,778 \\
& $k=3$ & 264,057 & 180,891 & 139,402 & 84,461 & 51,896 \\
& $k=4$ & 14707,132 & 10071,039 & 7759,992 & 4700,897 & 2888,180 \\
& Variância & 8,323 & 5,850 & 4,567 & 2,816 & 1,749 \\
& Assimetria & 10,117 & 12,059 & 13,645 & 17,376 & 22,053 \\
& Curtose & 200,249 & 282,506 & 360,321 & 581,168 & 932,944 \\
\hline 1,0 & $k=1$ & 0,466 & 0,338 & 0,268 & 0,170 & 0,108 \\
& $k=2$ & 0,823 & 0,575 & 0,447 & 0,274 & 0,170 \\
& $k=3$ & 2,320 & 1,600 & 1,237 & 0,752 & 0,463 \\
& $k=4$ & 9,001 & 6,180 & 4,767 & 2,892 & 1,778 \\
& Variância & 0,606 & 0,461 & 0,375 & 0,245 & 0,158 \\
& Assimetria & 2,910 & 3,499 & 3,983 & 5,117 & 6,528 \\
& Curtose & 15,282 & 20,592 & 25,695 & 40,280 & 63,560 \\
\hline 1,5 & $k=1$ & 0,448 & 0,333 & 0,268 & 0,175 & 0,113 \\
& $k=2$ & 0,527 & 0,376 & 0,296 & 0,185 & 0,116 \\
& $k=3$ & 0,823 & 0,575 & 0,447 & 0,274 & 0,170 \\
& $k=4$ & 1,576 & 1,091 & 0,844 & 0,514 & 0,317 \\
& Variância & 0,326 & 0,265 & 0,224 & 0,154 & 0,103 \\
& Assimetria & 1,579 & 2,003 & 2,343 & 3,116 & 4,051 \\
& Curtose & 5,784 & 7,658 & 9,518 & 14,905 & 23,517 \\
\hline
\end{tabular}

Se o parâmetro $\alpha$ assume o número racional, expresso como $k=p / q$, em que $p$ e $q$ são números inteiros, então a expressão (2.35) pode ser avaliada analiticamente, substituindo-se $t$ por $-t$. Desse modo obtemos

$$
M(t)=\sum_{m=1}^{\infty} \frac{m e^{\lambda} p_{m}}{(-t)^{\alpha}} \frac{p^{\alpha-1 / 2} q^{1 / 2}}{(\sqrt{2 \pi})^{q+p+2}} G_{p, q}^{m, n}\left(\begin{array}{cccc|c}
\frac{1-\alpha}{p}, & \frac{2-\alpha}{p}, & \cdots, & \frac{p-\alpha}{p} & \frac{p^{p}}{\left(q m^{-1} e^{-\lambda}(-t)^{\alpha}\right)^{q}}
\end{array}\right)
$$

em que $G$ é a Meijer G-função definida por

$$
G_{p, q}^{m, n}\left(\begin{array}{ccc}
a_{1}, & \cdots, & a_{p} \\
b_{1}, & \cdots, & b_{q}
\end{array} \mid x\right)=\frac{1}{2 \pi i} \int_{L} \frac{\prod_{m}^{j=1} \Gamma\left(b_{j}+t\right) \prod_{n}^{j=1} \Gamma\left(1-a_{j}-t\right)}{\prod_{j=n+1}^{p} \Gamma\left(a_{j}+t\right) \prod_{j=m+1}^{p} \Gamma\left(1-b_{j}-t\right)} x^{-t} d t
$$

\section{Estatísticas de ordem}

A função densidade $f_{i: n}(y)$ de $i$-ésima estatística de ordem para $i=1, \cdots, n$ correspondente à variável aleatória $Y_{1}, \cdots, Y_{n}$ seguindo a distribuição WBN-PA, pode ser 
escrita como

$$
f_{i: n}(y)=\frac{f(y)}{B(i, n-i+1)} \sum_{j=0}^{i-1}\left(\begin{array}{c}
i-1 \\
j
\end{array}\right)(-1)^{j}[1-F(y)]^{n+j-i}
$$

em que $B(a, b)=\Gamma(a+b) /(\Gamma(a) \Gamma(b))$ é uma função Beta, $f(y)$ e $F(y)$ são $p d f$ e $f d a$ da distribuição WBN-FA dada em (2.15) e (2.18) respectivamente. Considerando $u=$ $\exp \left(-y^{\alpha} e^{\lambda}\right)$, de $(2.16)$ e $(2.17)$ temos que

$$
f_{i: n}(y)=\frac{\alpha e^{\lambda}}{B(i, n-i+1)} \sum_{m=1}^{\infty} m p_{m} y^{\alpha-1} u^{m} \sum_{j=0}^{i-1}\left(\begin{array}{c}
i-1 \\
j
\end{array}\right)(-1)^{j}\left(\sum_{k=1}^{\infty} u^{k} p_{k}\right)^{n+j-i}
$$

e através da equação Gradshteyn \& Ryzhik (2000) para uma série de potências elevada a um número inteiro positivo j

$$
\left(\sum_{i=0}^{\infty} a_{i} y^{i}\right)^{j}=\sum_{i=0}^{\infty} c_{j, i} y^{i}
$$

cujo coeficiente $c_{j, i}=\left(i a_{0}\right)^{-1} \sum_{m=1}^{i}(j m-i+m) a_{m} c_{j, i-m}$, em que $c_{j, 0}=a_{0}^{j}$, logo, consequentemente os coeficientes $c_{j, i}$ podem ser obtidos. Usando esta equação e combinando seus termos, obtemos

$$
f_{i: n}(y)=\sum_{k, m=1}^{\infty} \sum_{j=0}^{i-1} \phi(k, m, j) g(y \mid \alpha, \lambda, m, k)
$$

em que $\phi(k, m, j)=\left(\begin{array}{c}i-1 \\ j\end{array}\right) \frac{(-1)^{j} m p_{m} c_{n+j-1, k}}{(m+k) B(i, n-i+1)}, g(y \mid \alpha, \lambda, m, k)$ tem distribuição de Weibull com parâmetro de escala $\lambda+\log (m+k)$, parâmetro de forma $\alpha$ e

$$
c_{j+n-1, k}=\left[k p_{1}\right]^{-1} \sum_{l=1}^{k}((n+j-1) l-k+l) c_{n+j-1, k-l} p_{l}
$$

com $c_{n+j-1,1}=p_{1}^{n+j-1}$. De (2.24) os momentos de estatísticas de ordem da distribuição WBN-PA podem ser escritos diretamente em termos dos momentos de Weibull como

$$
E\left[Y_{i: n}^{s}\right]=\sum_{k, m=1}^{\infty} \Gamma\left(\frac{s}{\alpha}+1\right) \sum_{j=0}^{i-1} \frac{\phi(k, m, j)}{\exp (-\lambda s / \alpha)(m+k)^{-s / \alpha}}
$$

Podemos calcular os momentos da estatística de ordem da distribuição WBN-PA pela equação (2.25) ou por integração numérica. Os valores numéricos dos momentos, variância, assimetria e curtose das estatísticas de ordem da distribuição WBN-PA são listados na 
Tabela 2.2 para $\lambda=0, \alpha=1,5, \eta=2, \theta=0,9, n=5$ e $s=3$.

TABELA 2.2: Momentos, variância, assimetria e curtose das estatísticas de ordem WBNPA para alguns valores selecionados

\begin{tabular}{lccccc}
\hline Estatística de ordem & $Y_{1: 5}$ & $Y_{2: 5}$ & $Y_{3: 5}$ & $Y_{4: 5}$ & $Y_{5: 5}$ \\
\hline$k=1$ & 0,1931 & 0,2083 & 0,1157 & 0,0344 & 0,0044 \\
$k=2$ & 0,0573 & 0,0837 & 0,0563 & 0,0192 & 0,0027 \\
$k=3$ & 0,0228 & 0,0411 & 0,0320 & 0,0122 & 0,0019 \\
$k=4$ & 0,0113 & 0,0239 & 0,0209 & 0,0087 & 0,0014 \\
Variância & 0,0201 & 0,0403 & 0,0429 & 0,0180 & 0,0027 \\
Assimetria & 1,3920 & 0,8514 & 1,7533 & 4,2564 & 13,2347 \\
Curtose & 5,8919 & 3,5597 & 5,4621 & 22,0399 & 195,5169 \\
\hline
\end{tabular}

\section{Entropia de Rényi}

A entropia de Rényi de uma variável aleatória $Y$ com a $f d p f(y)$ é uma medida da variação da incerteza definida por

$$
I_{R}(\rho)=\frac{1}{1-p} \log \left\{\int f(y)^{\rho} d y\right\}
$$

em que $f(y)$ é a $f d p$ de $Y, \rho>0$ e $\rho \neq 0$. Seja $Y$ a variável aleatória com a distribuição WBN-PA, usando expansão série de Taylor para $\left[1+\eta \theta\left(1-e^{-u}\right)\right]^{-\rho(1 / \eta+1)}$ e $\left(1-e^{u}\right)^{k}$, temos que

$$
\begin{aligned}
\int f(y)^{\rho} d y= & \left(\frac{\theta}{1-(1+\eta \theta)^{-1 / \eta}}\right)^{\rho} \alpha^{\rho-1} \exp \left(\frac{\lambda}{\alpha}(\rho-1)\right) \Gamma(A) \\
& \times \sum_{i, k=0}^{\infty} \frac{(\eta \theta)^{k}}{(\rho+i)^{A}}\left(\begin{array}{c}
\rho(1 / \eta+1)+k+1 \\
k
\end{array}\right)\left(\begin{array}{c}
k+i+1 \\
i
\end{array}\right),
\end{aligned}
$$

em que $A=\frac{\rho(\alpha-1)+1}{\alpha}$. Assim, de (2.26) e (2.27), a entropia de Rényi da distribuição WBN-PA é dada por

$$
\begin{aligned}
I_{R}^{f}(\rho)= & \frac{1}{1-\rho}\left\{\rho \log \left(\frac{\theta}{1-(1+\eta \theta)^{-1 / \eta}}\right)+(\rho-1)\left(\log \alpha+\frac{\lambda}{\alpha}\right)+\log \left[\Gamma\left(\frac{\rho(\alpha-1)+1}{\alpha}\right)\right]\right. \\
& \left.+\log \left[\sum_{i, k=0}^{\infty} \frac{(\eta \theta)^{k}}{(\rho+i)^{\frac{\rho(\alpha-1)+1}{\alpha}}}\left(\begin{array}{c}
\rho(1 / \eta)+k+1 \\
k
\end{array}\right)\left(\begin{array}{c}
k+i+1 \\
i
\end{array}\right)\right]\right\} .
\end{aligned}
$$




\subsubsection{A distribuição WBN sob esquema de última ativação}

Seja $Y$ uma variável aleatória não negativa que segue a distribuição WBN-UA com o vetor de parâmetros $\boldsymbol{\xi}=(\lambda, \alpha, \eta, \theta)$, a $f d p$ de $Y$, é dada por

$$
f(y ; \xi)=\frac{\theta \alpha y^{\alpha-1} \exp \left(\lambda-y^{\alpha} e^{\lambda}\right)\left(1+\eta \theta \exp \left(-y^{\alpha} e^{\lambda}\right)\right)^{-(1 / \eta+1)}}{1-(1+\eta \theta)^{-1 / \eta}}, \quad y>0
$$

Alguns sub-modelos da distribuição WBN-UA (2.28) são obtidos a seguir. Se $\eta=1$ temos a distribuição Weibull Geométrica sob última ativação (WG-UA); Se $\eta \rightarrow 0$, obtemos a distribuição Weibull Poisson sob última ativação (WP-UA). Fixando $\alpha=1$, quando $\eta=1$, temos a distribuição Exponencial-Geométrica proposta por Roman et al. (2011) e quando $\eta \rightarrow 0$, obtemos a distribuição Exponencial-Poisson introduzida por G. et al. (2011). Além disso, quando $\theta \rightarrow 0$ a distribuição WBN-UA (2.28) leva à distribuição de Weibull (1.1). A função densidade de probabilidade da distribuição WBN-UA é exibida na Figura 2.4 para $\lambda=0$, alguns valores do vetor $\phi=(\alpha, \theta)$ e $\eta=-1 ;-0,5 ; 0,01 ; 1,0$ e 10 .
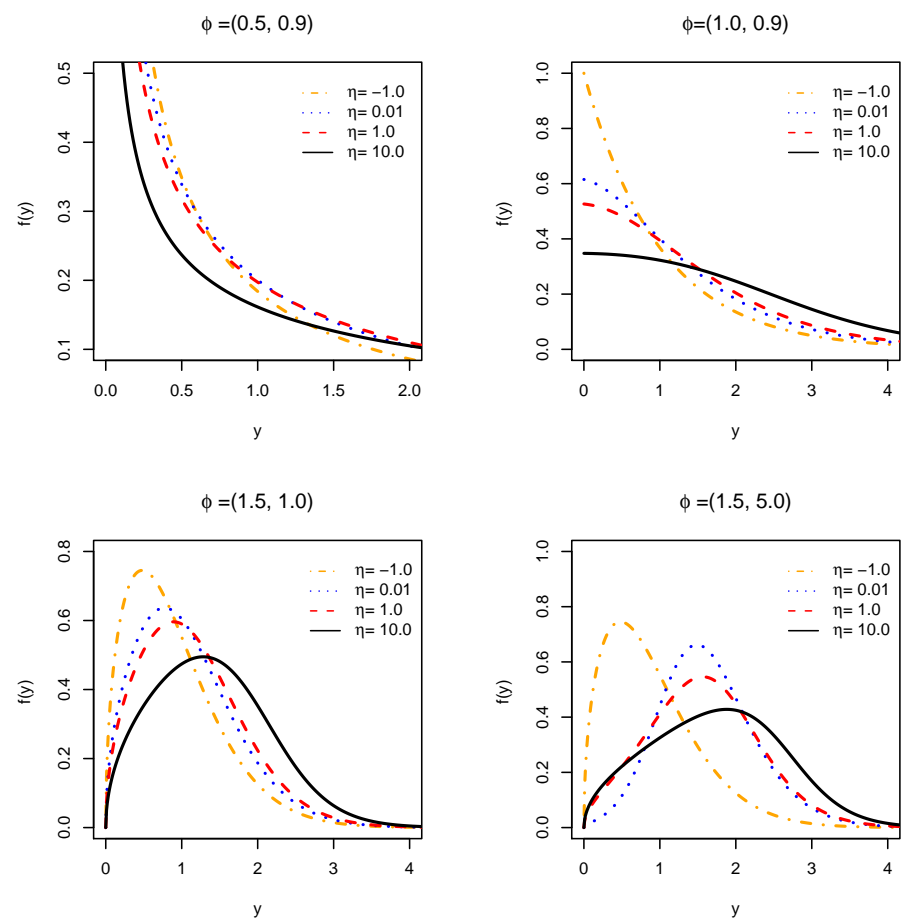

FIGURA 2.4: Função densidade de probabilidade da distribuição WBN-UA para alguns valores selecionados

Note que a $f d p$ da distribuição WBN-UA dada em (2.28) também pode ser escrita como

$$
f(y ; \xi)=\sum_{m=1}^{\infty} f(y \mid m, \alpha, \lambda) p_{m}
$$


em que $f(y \mid m, \alpha, \lambda)=\alpha m y^{\alpha-1} \exp \left(\lambda-y^{\alpha} e^{\lambda}\right)\left(1-\exp \left(-y^{\alpha} e^{\lambda}\right)\right)^{m-1}$ é a distribuição Weibull Exponenciada (WE) com parâmetros $\lambda, \alpha$ e $m$. Então a $f d a$ da distribuição WBN-UA tem expressão dada por

$$
F(y ; \xi)=\sum_{m=1}^{\infty}\left(1-\exp \left(-y^{\alpha} e^{\lambda}\right)\right)^{m} p_{m}
$$

Portanto, podemos obter algumas de suas propriedades matemáticas da distribuição WBN-UA a partir da distribuição WE.

\section{Propriedades da distribuição WBN-UA}

A $f d a$ da distribuição WBN-UA é dada por

$$
F(y ; \xi)=\frac{\left(1+\eta \theta e^{-(\beta y)^{\alpha}}\right)^{-1 / \eta}-(1+\eta \theta)^{-1 / \eta}}{1-(1+\eta \theta)^{-1 / \eta}}, \quad y>0
$$

As funções de sobrevivência e de risco de $Y$ são

$$
S(y ; \xi)=\frac{1-\left(1+\eta \theta \exp \left(-y^{\alpha} e^{\lambda}\right)\right)^{-1 / \eta}}{1-(1+\eta \theta)^{-1 / \eta}}, \quad y>0
$$

e

$$
h(y ; \xi)=\frac{\theta \alpha y^{\alpha-1} \exp \left(\lambda-y^{\alpha} e^{\lambda}\right)\left(1+\eta \theta \exp \left(-y^{\alpha} e^{\lambda}\right)\right)^{-(1 / \eta+1)}}{1-\left(1+\eta \theta \exp \left(-y^{\alpha} e^{\lambda}\right)\right)^{-1 / \eta}}, y>0,
$$

A função de risco (2.33) é decrescente para $0<\alpha<1$ e crescente para $\alpha \geq 1$. No entanto, para outros valores de parâmetros, pode assumir outras formas diferentes. A Figura 2.5 ilustra algumas das formas possíveis da função de risco para valores selecionados do vetor $\phi=(\alpha, \theta)$, $\eta=-1,0 ;-0,5 ; 0,01 ; 1,0 ; 10.0$ e $\lambda=0$. 

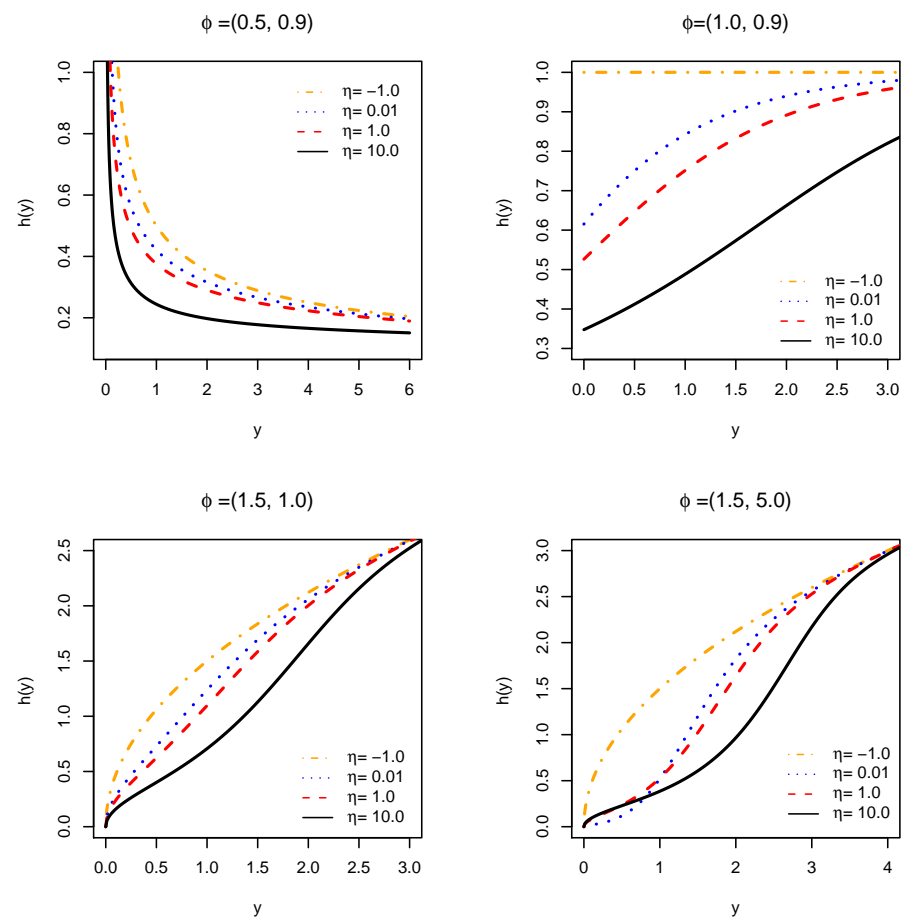

FIGURA 2.5: Função de risco da distribuição WBN-UA para alguns valores selecionados

\section{Quantis e momentos}

Os quantis $q$ da distribuição WBN-UA são obtidos invertendo a função densidade acumulada da distribuição WBN-UA dada por

$$
y_{q}=G^{-1}\left(\frac{1+\eta-\left[q\left(1-p_{0}\right)^{+} p_{0}\right]^{-\eta}}{\eta \theta}\right)
$$

Em particular, a mediana é dada por $y_{1 / 2}=G^{-1}\left(\frac{1+\eta-\left[\frac{1}{2}\left(1-p_{0}\right)^{+} p_{0}\right]^{-\eta}}{\eta \theta}\right)$, em que $G^{-1}(y)=$ $\left(-\log (1-y) / e^{\lambda}\right)^{1 / \alpha}$.

O k-ésimo momento ordinário de $Y$ é dado por

$$
E\left[Y^{k}\right]=\alpha e^{\lambda} \sum_{m=1}^{\infty} m \varphi(k, \alpha, \lambda, m) p_{m}
$$

no qual $\varphi(k, \alpha, \eta, \theta)=\int_{0}^{\infty} y^{k+\alpha-1} e^{-y^{\alpha} e^{\lambda}}\left(1-e^{-y^{\alpha} e^{\lambda}}\right)^{m-1} d_{y}$. Usando a expansão série de Taylor, a expressão (2.34) também pode ser escrita como

$$
E\left[Y^{k}\right]=\Gamma\left(\alpha^{-1}+k-1\right) \exp \left\{\lambda\left(2-k-\alpha^{-1}\right)\right\} \sum_{m=1}^{\infty} \sum_{j=0}^{m-1} m p_{m}(-1)^{j}\left(\begin{array}{c}
m-1 \\
j
\end{array}\right)(j+1)^{-\left(k-1+\alpha^{-1}\right)}
$$


Várias expressões, tais como média, variância, assimetria e curtose, podem ser obtidas numericamente por algum software matemático a partir de (2.34), tais como o Maple. A Tabela 2.3 apresenta os valores numéricos dos momentos ordinários, variância, assimetria e curtose da distribuição WBN-UA para $\lambda=0 ; \alpha=0,5 ; 1,0 ; 1,5$ e $\theta=0,9$.

TABELA 2.3: Momento ordinário, variância, assimetria e curtose da distribuição WBN-UA para alguns valores selecionados

\begin{tabular}{clccccc}
\hline$\alpha$ & & $\eta=0,01$ & $\eta=1$ & $\eta=2$ & $\eta=5$ & $\eta=10$ \\
\hline 0,5 & $k=1$ & 3,240 & 3,009 & 2,832 & 2,470 & 2,112 \\
& $k=2$ & 42,042 & 41,111 & 40,301 & 38,357 & 35,993 \\
& $k=3$ & 1287,034 & 1279,027 & 1271,536 & 1251,677 & 1223,533 \\
& $k=4$ & 72448,653 & 72329,104 & 72212,850 & 71886,022 & 71343,334 \\
& Variância & 31,544 & 32,059 & 32,281 & 32,253 & 31,531 \\
& Assimetria & 5,342 & 5,302 & 5,314 & 5,446 & 5,729 \\
& Curtose & 58,377 & 57,331 & 57,152 & 58,455 & 62,271 \\
\hline 1,0 & $k=1$ & 0,731 & 0,642 & 0,580 & 0,468 & 0,370 \\
& $k=2$ & 1,620 & 1,504 & 1,416 & 1,235 & 1,056 \\
& $k=3$ & 5,119 & 4,912 & 4,742 & 4,362 & 3,940 \\
& $k=4$ & 21,021 & 20,555 & 20,151 & 19,178 & 17,996 \\
& Variância & 1,086 & 1,092 & 1,079 & 1,016 & 0,919 \\
& Assimetria & 2,074 & 2,228 & 2,379 & 2,764 & 3,256 \\
& Curtose & 8,815 & 9,348 & 10,015 & 12,094 & 15,356 \\
\hline 1,5 & $k=1$ & 0,417 & 0,357 & 0,317 & 0,247 & 0,189 \\
& $k=2$ & 0,605 & 0,543 & 0,499 & 0,414 & 0,337 \\
& $k=3$ & 1,080 & 1,003 & 0,944 & 0,823 & 0,704 \\
& $k=4$ & 2,251 & 2,141 & 2,052 & 1,859 & 1,652 \\
& Variância & 0,432 & 0,416 & 0,399 & 0,353 & 0,302 \\
Assimetria & 1,651 & 1,909 & 2,120 & 2,603 & 3,177 \\
Curtose & 5,320 & 6,216 & 7,089 & 9,501 & 13,063 \\
\hline
\end{tabular}

\section{Função geradora de momentos}

A função geradora de momentos $(f g m) M(t)=E[\exp (t Y)]$, obtida a partir da expansão em série de potência para a função exponencial e também através de (2.29), é dada por

$$
\begin{aligned}
M(t) & =\int_{0}^{\infty} \exp (t y) f(y) d y \\
& =\sum_{m=1}^{\infty} \sum_{k=0}^{m-1}\left(\begin{array}{c}
m-1 \\
k
\end{array}\right) m p_{m}(-1)^{k} \alpha e^{\lambda} g(y, \alpha, \lambda, k, t)
\end{aligned}
$$

em que $g(y, \alpha, \lambda, k, t)=\int_{0}^{\infty} y^{\alpha-1} \exp \left(-y^{\alpha} e^{\lambda}(k+1)+t y\right) d y$. 


\section{Estatísticas de ordem}

A função densidade $f_{i: n}(y)$ de $i$-ésima estatística de ordem para $i=1, \cdots, n$ correspondente à variável aleatória $Y_{1}, \cdots, Y_{n}$ seguindo a distribuição WBN-UA, pode ser escrita como

$$
f_{i: n}(y)=\frac{f(y)}{B(i, n-i+1)} \sum_{j=0}^{i-1}\left(\begin{array}{c}
i-1 \\
j
\end{array}\right)(-1)^{j}[1-F(y)]^{n+j-i}
$$

em que $B(a, b)=\Gamma(a+b) /(\Gamma(a) \Gamma(b))$ é uma função Beta, $f(y)$ e $F(y)$ são $p d f$ e $f d a$ da distribuição WBN-UA dada em (2.28) e (2.31) respectivamente. Considerando $u=\exp \left(-y^{\alpha} e^{\lambda}\right)$, de $(2.29)$ e (2.30) temos que

$$
f_{i: n}(y)=\frac{\alpha e^{\lambda}}{B(i, n-i+1)} \sum_{m=1}^{\infty} m p_{m} y^{\alpha-1} u^{m-1} \sum_{j=0}^{i-1}(-1)^{j}\left(\begin{array}{c}
i-1 \\
j
\end{array}\right)\left[1-\sum_{k=1}^{\infty} u^{k} p_{k}\right]^{n+j-i} .
$$

Usando a equação de Gradshteyn \& Ryzhik (2000), obtemos

$$
f_{i: n}(y)=\sum_{k, m=1}^{\infty} \sum_{j=0}^{i-1} \sum_{z=0}^{\infty} \phi(k, m, j, z) g(y \mid \alpha, \lambda, m, k)
$$

em que $\phi(k, m, j)=\left(\begin{array}{c}i-1 \\ j\end{array}\right)\left(\begin{array}{c}n+j-1 \\ z\end{array}\right) \frac{(-1)^{j} m p_{m} c_{z, k}}{(m+k-1) B(i, n-i+1)}, c_{z, k}=\left[k p_{1}\right]^{-1} \sum_{m=1}^{k}(z m-k+m) c_{z, k-m} p_{m}$ $\operatorname{com} c_{z, 1}=p_{1}^{z}$ e $g(y \mid \alpha, \lambda, m, k)$ tem distribuição de Weibull com parâmetro de escala $\lambda+\log (m+$ $k-1)$, parâmetro de forma $\alpha$. De (2.36) os momentos de estatísticas de ordem da distribuição WBN-UA podem ser escritos diretamente em termos dos momentos de Weibull, como

$$
E\left[Y_{i: n}^{s}\right]=\sum_{k, m=1}^{\infty} \sum_{j=0}^{i-1} \sum_{z=0}^{\infty} \frac{\phi(k, m, j, z)}{\exp (-\lambda s / \alpha)(m+k-1)^{-s / \alpha}}
$$

Podemos calcular os momentos de estatísticas de ordem da distribuição WBN-UA pela equação (2.37) ou por integração numérica. Apresentamos os valores numéricos dos momentos, variância, assimetria e curtose das estatísticas de ordem da distribuição WBN-UA para $\lambda=0$; $\alpha=1,5 ; \eta=2 ; \theta=0.9 ; n=5$ e $s=3$, na Tabela 2.4. 
TABELA 2.4: Momentos, variância, assimetria e curtose das estatísticas de ordem da distribuição WBN-UA para alguns valores selecionados

\begin{tabular}{lccccc}
\hline Estatísticas de ordem & $Y_{1: 5}$ & $Y_{2: 5}$ & $Y_{3: 5}$ & $Y_{4: 5}$ & $Y_{5: 5}$ \\
\hline$k=1$ & 0,4672 & 0,4749 & 0,2518 & 0,0721 & 0,0088 \\
$k=2$ & 0,3036 & 0,3993 & 0,2473 & 0,0789 & 0,0105 \\
$k=3$ & 0,2412 & 0,3799 & 0,2653 & 0,0924 & 0,0131 \\
$k=4$ & 0,2210 & 0,3987 & 0,3067 & 0,1149 & 0,0173 \\
Variância & 0,0853 & 0,1738 & 0,1839 & 0,0737 & 0,0104 \\
Assimetria & 0,7868 & 0,3480 & 1,4006 & 3,8049 & 12,1347 \\
Curtose & 3,4247 & 2,1443 & 3,5915 & 16,7051 & 156,1324 \\
\hline
\end{tabular}

\section{Entropia de Rényi}

Usando as mesmas ideias da Seção 2.2.1, a entropia de Rényi definida em (2.26) da variável aleatória $Y$ com a distribuição WBN-UA é dada por

$$
\begin{aligned}
I_{R}(\rho)= & \frac{1}{1-\rho}\left\{\rho \log \left(\frac{\theta}{1-(1+\eta \theta)^{-1 / \text { eta }}}\right)+(\rho-1)\left(\log \alpha+\frac{\lambda}{\alpha}\right)+\log \left[\Gamma\left(\frac{\rho(\alpha-1)+1}{\alpha}\right)\right]\right. \\
& \left.\log \left[\sum_{k=0}^{\infty}(-1)^{k} \frac{(\eta \theta)^{k}}{(\rho+k)^{\frac{\rho(\alpha-1)+1}{\alpha}}}\left(\begin{array}{c}
\rho(1 / \eta+1) \\
k
\end{array}\right)\right]\right\} .
\end{aligned}
$$

\subsection{Inferência Clássica}

Seja $y_{1}, \cdots, y_{n}$ uma amostra aleatória de tamanho $n$ da distribuição WBN-PA com vetor de parâmetros $\boldsymbol{\xi}=(\alpha, \lambda, \eta, \theta)^{\top}$. A função de log-verossimilhança para $\boldsymbol{\xi}$ pode ser escrita como

$$
\begin{aligned}
\ell= & n\left[\log \theta+\log \alpha+\lambda-\log \left(1-(1+\eta \theta)^{-1 / \eta}\right)\right]+(\alpha-1) \sum_{i=1}^{n} \log y_{i}-e^{\lambda} \sum_{i=1}^{n} y_{i}^{\alpha} \\
& -\left(\frac{1}{\eta}+1\right) \sum_{i=1}^{n} \log \left[1+\eta \theta\left(1-\exp \left(-y_{i}^{\alpha} e^{\lambda}\right)\right)\right]
\end{aligned}
$$


Os componentes do vetor escore $U(\boldsymbol{\xi})=(\partial \ell / \partial \alpha, \partial \ell / \partial \lambda, \partial \ell / \partial \eta, \partial \ell / \partial \theta)^{\top}$ são dados por

$$
\begin{aligned}
\frac{\partial \ell}{\partial \alpha} & =\frac{n}{\alpha}+\sum_{i=1}^{n} \log y_{i}-e^{\lambda} \sum_{i=1}^{n} y_{i}^{\alpha} \log y_{i}-(\eta+1) \sum_{i=1}^{n} \theta e^{\lambda} T_{1,1,1,1}^{(i)} \\
\frac{\partial \ell}{\partial \lambda} & =n-e^{\lambda} \sum_{i=1}^{n} y_{i}^{\alpha}-(1+\eta) \sum_{i=1}^{n} \theta e^{\lambda} T_{1,0,1,1}^{(i)} \\
\frac{\partial \ell}{\partial \theta} & =\frac{n \theta(1+\eta \theta)^{-1 / \eta} \log (1+\eta \theta)}{\eta^{2}\left(1-(1+\eta \theta)^{-1 / \eta}\right)}+\sum_{i=1}^{n} \frac{1}{\eta^{2}} \log \left(T_{0,0,-1,0}^{(i)}\right)-\left(1+\frac{1}{\eta^{2}}\right) \theta\left(T_{0,0,1,0}^{(i)}-T_{0,0,1,1}^{(i)}\right) \\
\frac{\partial \ell}{\partial \eta} & =\frac{n}{\theta}-\frac{n(1+\eta \theta)^{-1 / \eta+1}}{1-(1+\eta \theta)^{-1 / \eta}}-(1+\eta) \sum_{i=1}^{n}\left(T_{0,0,1,0}^{(i)}-T_{0,0,1,1}^{(i)}\right)
\end{aligned}
$$

em que $T_{j, k, l, m}^{(i)}=y_{i}^{\alpha j}\left\{\log y_{i}\right\}^{k}\left(1+\eta \theta\left(1-\exp \left(-y_{i}^{\alpha} e^{\lambda}\right)\right)\right)^{-l} \exp \left(-y_{i}^{\alpha} e^{\lambda}\right)^{m}$, para $j, k, l, m \in\{0,1,2\}$ e $i=1 \cdots n$.

O estimador de máxima verossimilhança (EMV) $\hat{\boldsymbol{\xi}}$ para o vetor de parâmetros $\boldsymbol{\xi}$ pode ser obtido da solução do sistema de equações $U(\boldsymbol{\xi})=0$. Geralmente, como esse sistema é não linear, as soluções do sistema são obtidas numericamente usando algum método de otimização, tais como Newton-Raphson.

No caso em que o tamanho da amostra é grande e sob certas condições de regularidade para a função de verossimilhança, o intervalo de confiança e o teste de hipóteses podem ser obtidos considerando o fato de que a distribuição assintótica para os EMV é a distribuição normal com média $\boldsymbol{\xi}$, e que a matriz de covariância é dada pelo inverso da matriz de informação de Fisher. Portanto, a matriz de covariância assintótica é dada por $I^{-1}(\boldsymbol{\xi}) \operatorname{com} I(\boldsymbol{\xi})=E\left[-\left\{\frac{\partial^{2} \ell(\boldsymbol{\xi})}{\partial \boldsymbol{\xi} \partial \boldsymbol{\xi} \uparrow}\right\}\right]$.

Mas em muitas situações a matriz de informação de Fisher não tem forma fechada e é dificilmente calculada. Nesses casos, pode-se usar a negativa da matriz hessiana da função de log-verossimilhança, denotada por $\ddot{L}(\boldsymbol{\xi})=-\left\{\frac{\partial^{2} \ell(\boldsymbol{\xi})}{\partial \boldsymbol{\xi} \partial \boldsymbol{\xi} \top}\right\}$, avaliada em $\boldsymbol{\xi}=\hat{\boldsymbol{\xi}}$, que é um estimador consistente para matriz de covariância assintótica (Mudholkar et al., 1996). Assim, a distribuição assintótica para $\hat{\boldsymbol{\xi}}$ é dada por $\boldsymbol{\xi} \sim N_{(q)}\left\{\boldsymbol{\xi} ; \ddot{L}(\boldsymbol{\xi})^{-1}\right\}$ em que $q$ é o número de parâmetros do modelo e $\ddot{L}(\boldsymbol{\xi})$ é conhecido como a matriz de informação observada.

Assim, a matriz de informação observada $\ddot{L}(\boldsymbol{\xi})=J_{4}(\boldsymbol{\xi})$ para $\boldsymbol{\xi}$ pode ser expressa como

$$
J_{4}(\boldsymbol{\xi})=\left(\begin{array}{cccc}
J_{\alpha \alpha} & J_{\alpha \lambda} & J_{\alpha \eta} & J_{\alpha \theta} \\
\cdot & J_{\lambda \lambda} & J_{\lambda \eta} & J_{\lambda \theta} \\
\cdot & \cdot & J_{\eta \eta} & J_{\eta \theta} \\
\cdot & \cdot & \cdot & J_{\theta \theta}
\end{array}\right)
$$


que é uma matriz simétrica, e seus componentes são dados por

$$
\begin{aligned}
& -J_{\alpha \alpha}=-\frac{n}{\alpha^{2}}-e^{\lambda} \sum_{i=1}^{n} y_{i}\left(\log y_{i}\right)^{2}+(\eta+1) \theta e^{2 \lambda} \sum_{i=1}^{n} y_{i}\left(T_{1,2,1,1}^{(i)}-\eta \theta T_{1,2,2,2}^{(i)}\right), \\
& -J_{\alpha \lambda}=-e^{\lambda} \sum_{i=1}^{n} y_{i}^{\alpha} \log y_{i}-(\eta+1) \theta e^{\lambda} \sum_{i=1}^{n} y_{i}\left(T_{0,1,1,1}^{(i)}-e^{\lambda} T_{1,1,1,1}^{(i)}-\eta \theta e^{\lambda} T_{2,1,2,2}^{(i)}\right), \\
& -J_{\alpha \eta}=-\theta(\eta+1) e^{\lambda} \sum_{i=1}^{n} y_{i}\left[T_{0,1,1,1}^{(i)}-(\eta+1) \theta\left(T_{0,1,2,1,}^{(i)}-T_{0,1,2,2}^{(i)}\right)\right] \\
& -J_{\alpha \theta}=-(\eta+1) e^{\lambda} \sum_{i=1}^{n} y_{i}\left(T_{0,1,1,1}^{(i)}-\eta \theta\left(T_{0,1,2,1}^{(i)}-T_{0,1,2,2}^{(i)}\right)\right) \\
& -J_{\lambda \lambda}=-e^{\lambda} \sum_{i=1}^{n} y_{i}^{\alpha}-(\eta+1) \theta \sum_{i=1}^{n} e^{\lambda}\left(T_{1,0,1,1}^{(i)}-e^{\lambda} T_{2,0,1,1}^{(i)}\right)-\eta \theta\left(T_{1,0,2,1}^{(i)}-e^{\lambda} T_{2,0,2,2}^{(i)}\right), \\
& -J_{\lambda \eta}=-\theta e^{\lambda} \sum_{i=1}^{n} T_{1,0,1,1}^{(i)}-\theta(\eta+1)\left(T_{1,0,2,1}^{(i)}-T_{1,0,2,2}^{(i)}\right) \text {, } \\
& -J_{\lambda \theta}=-(\eta+1) e^{\lambda} \sum_{i=1}^{n} T_{1,0,1,1}^{(i)}-\eta \theta\left(T_{1,0,2,1}^{(i)}-T_{1,0,2,2}^{(i)}\right), \\
& -J_{\eta \eta}=\frac{n \theta^{2}}{\eta^{2}\left[(1+\eta \theta)^{-1 / \eta}-1\right]}+\frac{n \theta \log (1+\eta \theta)\left[(1+\eta \theta)^{1 / \eta}(2 \eta-\theta \log (1+\eta \theta))-2 \eta\right]}{\eta^{4}\left[(1+\eta \theta)^{1 / \eta}-1\right]^{2}} \\
& +\frac{1}{\eta^{3}} \sum_{i=1}^{n} \theta(\eta-1)\left(T_{0,0,1,0}^{(i)}-T_{0,0,1,1}^{(i)}\right)-2 \log T_{0,0,1,0}^{(i)}+\left(1+1 / \eta^{2}\right) \theta^{2} \sum_{i=1}^{n} T_{0,0,2,0}^{(i)} \\
& -2 T_{0,0,2,1}^{(i)}+T_{0,0,2,2}^{(i)} \\
& -J_{\eta \theta}=\frac{n \theta\left(\eta(1+\eta \theta)^{-1 / \eta}+1\right) \log (1+\eta \theta)}{\eta^{2}\left((1+\eta \theta)^{-1 / \eta}-1\right)^{2}(1+\eta \theta)}-\left(1-\frac{1}{\eta}+\frac{1}{\eta^{2}}\right) \sum_{i=1}^{n} T_{0,0,1,0}^{(i)}-T_{0,0,1,1}^{(i)} \\
& -\theta\left(\eta+\frac{1}{\eta}\right) \sum_{i=1}^{n} T_{0,0,2,0}^{(i)}-2 T_{0,0,2,1}^{(i)}+T_{0,0,2,2}^{(i)}, \\
& -J_{\theta \theta}=-\frac{n}{\theta^{2}}+\frac{n(1+\eta \theta)^{-(2 / \eta+2)}\left[(1+\eta)(1+\eta \theta)^{1 / \eta}\left(1-(1+\eta \theta)^{-1 / \eta}\right)+1\right]}{\left(1-(1+\eta \theta)^{-1 / \eta}\right)^{2}} \\
& +\eta(\eta+1) \sum_{i=1}^{n} T_{0,0,2,0}^{(i)}-2 T_{0,0,2,1}^{(i)}+T_{0,0,2,2}^{(i)} .
\end{aligned}
$$

Agora, seja $y_{1}, \cdots, y_{n}$ uma amostra aleatória de tamanha $n$ da distribuição WBN-UA com vetor de parâmetros $\boldsymbol{\xi}=(\alpha, \lambda, \eta, \theta)$. A função de log-verossimilhança para $\boldsymbol{\xi}$ pode escrito como

$$
\begin{aligned}
\ell= & n\left[\log \theta+\log \alpha+\lambda-\log \left(1-(1+\eta \theta)^{-1 / \eta}\right)\right]+(\alpha-1) \sum_{i=1}^{n} \log y_{i}-e^{\lambda} \sum_{i=1}^{n} y_{i}^{\alpha} \\
& -\left(\frac{1}{\eta}+1\right) \sum_{i=1}^{n} \log \left[1+\eta \theta \exp \left(-y_{i}^{\alpha} e^{\lambda}\right)\right] .
\end{aligned}
$$


Os componentes do vetor escore $U(\boldsymbol{\xi})=(\partial \ell / \partial \alpha, \partial \ell / \partial \lambda, \partial \ell / \partial \eta, \partial \ell / \partial \theta)^{\top}$ são dados por

$$
\begin{aligned}
\frac{\partial \ell}{\partial \alpha} & =\frac{n}{\alpha}+\sum_{i=1}^{n} \log y_{i}-e^{\lambda} \sum_{i=1}^{n} y_{i}^{\alpha} \log y_{i}+(\eta+1) \sum_{i=1}^{n} \theta e^{\lambda} S_{1,1,1,1}^{(i)} \\
\frac{\partial \ell}{\partial \lambda} & =n-e^{\lambda} \sum_{i=1}^{n} y_{i}^{\alpha}+(1+\eta) \sum_{i=1}^{n} \theta e^{\lambda} S_{1,0,1,1}^{(i)} \\
\frac{\partial \ell}{\partial \theta} & =\frac{n \theta(1+\eta \theta)^{-1 / \eta} \log (1+\eta \theta)}{\eta^{2}\left(1-(1+\eta \theta)^{-1 / \eta}\right)}+\sum_{i=1}^{n} \frac{1}{\eta^{2}} \log \left(S_{0,0,-1,0}^{(i)}\right)-\left(1+\frac{1}{\eta^{2}}\right) \theta\left(S_{0,0,1,0}^{(i)}-S_{0,0,1,1}^{(i)}\right) \\
\frac{\partial \ell}{\partial \eta} & =\frac{n}{\theta}-\frac{n(1+\eta \theta)^{-1 / \eta+1}}{1-(1+\eta \theta)^{-1 / \eta}}-(1+\eta) \sum_{i=1}^{n}\left(S_{0,0,1,0}^{(i)}-S_{0,0,1,1}^{(i)}\right)
\end{aligned}
$$

em que $S_{j, k, l, m}^{(i)}=y_{i}^{\alpha j}\left\{\log y_{i}\right\}^{k}\left(1+\eta \theta \exp \left(-y_{i}^{\alpha} e^{\lambda}\right)\right)^{-l} \exp \left(-y_{i}^{\alpha} e^{\lambda}\right)^{m}$, for $j, k, l, m \in\{0,1,2\}$ e $i=$ $1 \cdots n$.

Nesse caso, a matriz de informação observada $\ddot{L}(\boldsymbol{\xi})=J_{4}(\boldsymbol{\xi})$ dada em (2.38) tem os componentes dados por

$$
\begin{aligned}
-J_{\alpha \alpha}= & -\frac{n}{\alpha^{2}}-e^{\lambda} \sum_{i=1}^{n} y_{i}\left(\log y_{i}\right)^{2}+(\eta+1) \theta e^{2 \lambda} \sum_{i=1}^{n} y_{i}\left(S_{1,2,1,1}^{(i)}+\eta \theta S_{1,2,2,2}^{(i)}\right), \\
-J_{\alpha \lambda}= & -e^{\lambda} \sum_{i=1}^{n} y_{i}^{\alpha} \log y_{i}-(\eta+1) \theta e^{\lambda} \sum_{i=1}^{n} y_{i}\left(S_{0,1,1,1}^{(i)}-e^{\lambda} S_{1,1,1,1}^{(i)}+\eta \theta e^{\lambda} S_{2,1,2,2}^{(i)}\right), \\
-J_{\alpha \eta}= & -\theta(\eta+1) e^{\lambda} \sum_{i=1}^{n} y_{i}\left[S_{0,1,1,1}^{(i)}-(\eta+1) \theta\left(S_{0,1,2,1,}^{(i)}-S_{0,1,2,2}^{(i)}\right)\right] \\
-J_{\alpha \theta}= & -(\eta+1) e^{\lambda} \sum_{i=1}^{n} y_{i}\left(S_{0,1,1,1}^{(i)}-\eta \theta\left(S_{0,1,2,1}^{(i)}-S_{0,1,2,2}^{(i)}\right)\right) \\
-J_{\lambda \lambda}= & -e^{\lambda} \sum_{i=1}^{n} y_{i}^{\alpha}+(\eta+1) \theta \sum_{i=1}^{n} e^{\lambda}\left(S_{1,0,1,1}^{(i)}-e^{\lambda} S_{2,0,1,1}^{(i)}\right)+\eta \theta\left(S_{1,0,2,1}^{(i)}-e^{\lambda} S_{2,0,2,2}^{(i)}\right), \\
-J_{\lambda \eta}= & \theta e^{\lambda} \sum_{i=1}^{n} S_{1,0,1,1}^{(i)}-\theta(\eta+1)\left(S_{1,0,2,1}^{(i)}-S_{1,0,2,2}^{(i)}\right), \\
-J_{\lambda \theta}= & (\eta+1) e^{\lambda} \sum_{i=1}^{n} S_{1,0,1,1}^{(i)}-\eta \theta\left(S_{1,0,2,1}^{(i)}-S_{1,0,2,2}^{(i)}\right), \\
-J_{\eta \eta}= & \frac{n \theta^{2}}{\eta^{2}\left[(1+\eta \theta)^{-1 / \eta}-1\right]}+\frac{n \theta \log (1+\eta \theta)\left[(1+\eta \theta)^{1 / \eta}(2 \eta-\theta \log (1+\eta \theta))-2 \eta\right]}{\eta^{4}\left[(1+\eta \theta)^{1 / \eta}-1\right]^{2}} \\
& +\frac{1}{\eta^{3} \sum_{i=1}^{n} \theta(\eta-1)\left(S_{0,0,1,0}^{(i)}-S_{0,0,1,1}^{(i)}\right)-2 \log S_{0,0,1,0}^{(i)}+\left(1+1 / \eta^{2}\right) \theta^{2} \sum_{i=1}^{n} S_{0,0,2,0}^{(i)}} \\
& -2 S_{0,0,2,1}^{(i)}+S_{0,0,2,2}^{(i)},
\end{aligned}
$$




$$
\begin{aligned}
-J_{\eta \theta}= & \frac{n \theta\left(\eta(1+\eta \theta)^{-1 / \eta}+1\right) \log (1+\eta \theta)}{\eta^{2}\left((1+\eta \theta)^{-1 / \eta}-1\right)^{2}(1+\eta \theta)}-\left(1-\frac{1}{\eta}+\frac{1}{\eta^{2}}\right) \sum_{i=1}^{n} S_{0,0,1,0}^{(i)}-S_{0,0,1,1}^{(i)} \\
& -\theta\left(\eta+\frac{1}{\eta}\right) \sum_{i=1}^{n} S_{0,0,2,0}^{(i)}-2 S_{0,0,2,1}^{(i)}+S_{0,0,2,2}^{(i)}, \\
-J_{\theta \theta}= & -\frac{n}{\theta^{2}}+\frac{n(1+\eta \theta)^{-(2 / \eta+2)}\left[(1+\eta)(1+\eta \theta)^{1 / \eta}\left(1-(1+\eta \theta)^{-1 / \eta}\right)+1\right]}{\left(1-(1+\eta \theta)^{-1 / \eta}\right)^{2}} \\
& +\eta(\eta+1) \sum_{i=1}^{n} S_{0,0,2,0}^{(i)}-2 S_{0,0,2,1}^{(i)}+S_{0,0,2,2}^{(i)} .
\end{aligned}
$$

$\mathrm{Na}$ análise de dados de sobrevivência, os tempos de falha $y_{i}$ geralmente não são observados completamente e são sujeitos à censura à direita. Seja $z_{i}$ o tempo observado tal que $z_{i}=\min \left\{y_{i}, c_{i}\right\}$, com $c_{i}$ sendo o tempo de censura e $\delta_{i}=I\left(y_{i} \leq c_{i}\right)$ sendo um indicador de censura, i.e., quando $\delta_{i}=1$ se $z_{i}$ é o tempo de falha e $\delta_{i}=0$ se ele é censurado à direita, para $i=1, \cdots, n$. Então, a função de verossimilhança para o vetor dos parâmetros $\boldsymbol{\xi}=(\alpha, \lambda, \eta, \theta)$ na censura não-informativa é dada por

$$
L(\xi) \propto \prod_{i=1}^{n}\left[f\left(z_{i} ; \xi\right)\right]^{\delta_{i}}\left[S\left(z_{i} ; \xi\right)\right]^{1-\delta_{i}}
$$

em que $f(\cdot, \xi)$ e $S(\cdot, \xi)$ são funções de densidade e sobrevivência da distribuição WBN sob diferentes esquemas de ativação, que foram apresentadas na Seção 2.2.

O teste da razão de verossimilhança (RV) é usado para comparar a distribuição WBN com alguns submodelos dele. Consideramos a partição $\xi=\left(\xi_{1}^{T}, \xi_{2}^{T}\right)$, em que $\xi_{1}$ é o subconjunto dos parâmetros de interesse e $\xi_{2}$ é o subconjunto dos parâmetros restantes. O teste da RV para a hipótese nula $H_{0}: \xi_{1}=\xi_{1}^{(0)}$ versus a hipótese alternativa $H_{1}: \xi_{1} \neq \xi_{1}^{(0)}$ é dado por $\omega=2\{\ell(\hat{\xi})-\ell(\widetilde{\xi})\}$, nos quais $\hat{\xi}$ e $\widetilde{\xi}$ são os EMV sob as hipóteses nula e alternativa, respectivamente. A estatística $\omega$ é distribuída assintoticamente (quando $n \rightarrow \infty$ ) e tem a distribuição $\chi_{k}^{2}$, em que $k$ é a dimensão do subconjunto de interesse $\xi_{1}$.

Os diferentes modelos podem ser comparados usando o critério de informação Akaike dado por $A I C=-2 \ell(\hat{\xi})+2 \#(\xi)$ e o critério de Schwartz-Bayesian definido por $S B C=-2 \ell(\hat{\xi})+$

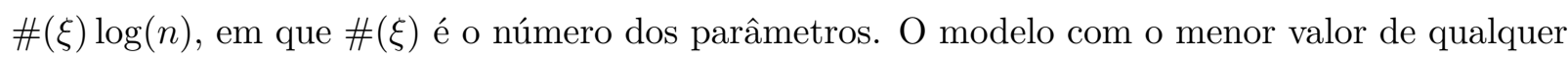
um desses critérios (entre todos os modelos considerados) é geralmente o melhor modelo para descrever o comportamento aleatório do conjunto de dados. 


\subsection{Simulação}

Um estudo de simulação foi realizado com objetivo de comparar os modelos propostos WBN na presença do esquema de primeira ativação (WBN-PA), última ativação (WBN-UA) e ativação aleatória (WBN-AA).

Os dados de tempo de falha foram simulados a partir da função quantil da distribuição (via método de inversão) com os parâmetros $\alpha=2, \lambda=-3, \eta=15$ e $\theta=50$. Os tempos de censura foram gerados a partir da distribuição uniforme $[0, \tau]$, em que $\tau$ controla a proporção de censura. Na simulação, consideramos $0 \%$ e $10 \%$ de observações censuradas, sendo o tamanho da amostra $N=50,100$ e 200. Para cada configuração, realizamos 1.000 réplicas e ajustamos por três modelos (WBN-PA, WBN-UA e WBN-AA), calculamos a estimativa da função de sobrevivência no ponto médio, seu erro padrão (EP) e a raiz quadrada do erro quadrático médio(RQEM). Além disso, calculamos a porcentagem de amostras que foram geradas pela distribuição proposta e que se mostraram como o melhor modelo ajustado de acordo com o critério AIC. Note que é possível discriminar as distribuições concorrentes pelo critério AIC.

A Tabela 2.6 apresenta as porcentagens das amostras que indicam o melhor modelo ajustado de acordo com os critérios da AIC, sendo que a medida aumenta com o aumento do tamanho da amostra; isso também acontece quando a amostra contem uma porcentagem das observações censuradas. Além disso, o valor de RQEM do melhor modelo sempre é menor comparado com as outras distribuições, o que pode ser observado na Tabela 2.5. 
TABELA 2.5: Estimativas da função de sobrevivência na mediana, EP e RQEM

\begin{tabular}{|c|c|c|c|c|c|c|c|c|}
\hline \multirow[b]{2}{*}{ Model } & \multirow[b]{2}{*}{$\mathrm{N}$} & \multirow[b]{2}{*}{ Model } & \multicolumn{3}{|c|}{$0 \%$ of censored } & \multicolumn{3}{|c|}{$10 \%$ of censored } \\
\hline & & & $\hat{S}\left(y_{\text {med }}\right)$ & $\mathrm{EP}$ & RQEM & $\hat{S}\left(y_{\text {med }}\right)$ & $\mathrm{EP}$ & RQEM \\
\hline \multirow{9}{*}{ WBN-PA } & 50 & WBN-PA & 0,553 & 0,0426 & 0,0678 & 0,580 & 0,0455 & 0,0918 \\
\hline & & WBN-UA & 0,592 & 0,0531 & 0,4085 & 0,621 & 0,0518 & 0,3799 \\
\hline & & WBN-AA & 0,613 & 0,0362 & 0,3648 & 0,639 & 0,0360 & 0,3386 \\
\hline & 100 & WBN-PA & 0,543 & 0,0300 & 0,0527 & 0,572 & 0,0294 & 0,0778 \\
\hline & & WBN-UA & 0,573 & 0,0444 & 0,4264 & 0,605 & 0,0418 & 0,3941 \\
\hline & & WBN-AA & 0,601 & 0,0273 & 0,3764 & 0,629 & 0,0248 & 0,3475 \\
\hline & 200 & WBN-PA & 0,515 & 0,0259 & 0,0239 & 0,535 & 0,0272 & 0,0339 \\
\hline & & WBN-UA & 0,519 & 0,0282 & 0,4860 & 0,541 & 0,0381 & 0,4062 \\
\hline & & WBN-AA & 0,568 & 0,0246 & 0,4087 & 0,618 & 0,0246 & 0,3387 \\
\hline \multirow[t]{9}{*}{ WBN-UA } & 50 & WBN-PA & 0,469 & 0,0595 & 0,4702 & 0,471 & 0,0593 & 0,4720 \\
\hline & & WBN-UA & 0,525 & 0,0534 & 0,0591 & 0,525 & 0,0566 & 0,0617 \\
\hline & & WBN-AA & 0,472 & 0,0569 & 0,4519 & 0,474 & 0,0576 & 0,4533 \\
\hline & 100 & WBN-PA & 0,460 & 0,0364 & 0,4583 & 0,462 & 0,0402 & 0,4611 \\
\hline & & WBN-UA & 0,515 & 0,0354 & 0,0385 & 0,518 & 0,0386 & 0,0427 \\
\hline & & WBN-AA & 0,460 & 0,0364 & 0,4372 & 0,463 & 0,0400 & 0,4403 \\
\hline & 200 & WBN-PA & 0,455 & 0,0235 & 0,4529 & 0,455 & 0,0267 & 0,4533 \\
\hline & & WBN-UA & 0,512 & 0,0227 & 0,0255 & 0,513 & 0,0256 & 0,0287 \\
\hline & & WBN-AA & 0,455 & 0,0235 & 0,4318 & 0,455 & 0,0267 & 0,4322 \\
\hline \multirow[t]{9}{*}{ WBN-AA } & 50 & WBN-PA & 0,463 & 0,0618 & 0,3826 & 0,470 & 0,0634 & 0,3901 \\
\hline & & WBN-UA & 0,500 & 0,0631 & 0,4197 & 0,506 & 0,0663 & 0,4142 \\
\hline & & WBN-AA & 0,480 & 0,0558 & 0,0593 & 0,485 & 0,0589 & 0,0609 \\
\hline & 100 & WBN-PA & 0,472 & 0,0447 & 0,3890 & 0,471 & 0,0440 & 0,3883 \\
\hline & & WBN-UA & 0,502 & 0,0508 & 0,4160 & 0,500 & 0,0493 & 0,4174 \\
\hline & & WBN-AA & 0,479 & 0,0414 & 0,0464 & 0,480 & 0,0409 & 0,0455 \\
\hline & 200 & WBN-PA & 0,446 & 0,0140 & 0,3608 & 0,474 & 0,0314 & 0,3904 \\
\hline & & WBN-UA & 0,496 & 0,0260 & 0,4194 & 0,496 & 0,0417 & 0,4214 \\
\hline & & WBN-AA & 0,446 & 0,0140 & 0,0552 & 0,478 & 0,0301 & 0,0372 \\
\hline
\end{tabular}

TABELA 2.6: Porcentagem de amostras em que o modelo ajustado foi indicado como o melhor modelo de acordo com o critério AIC

\begin{tabular}{ccrrrrrr} 
Modelo & N & \multicolumn{3}{c}{ \% of censored } & \multicolumn{3}{c}{$10 \%$ of censored } \\
\cline { 3 - 7 } WBN-PA & 50 & 71,1 & 0,2 & 28,7 & 62,1 & 1,5 & 36,4 \\
& 100 & 92,9 & 0,0 & 7,1 & 88,6 & 0,6 & 10,8 \\
& 200 & 100,0 & 0,0 & 0,0 & 97,0 & 0,2 & 2,8 \\
\hline WBN-UA & 50 & 0,1 & 60,9 & 39,0 & 0,4 & 53,0 & 46,6 \\
& 100 & 0,0 & 84,7 & 15,3 & 0,0 & 81,9 & 18,1 \\
& 200 & 0,0 & 96,6 & 3,4 & 0,0 & 98,0 & 2,0 \\
\hline WBN-AA & 50 & 5,5 & 7,2 & 87,3 & 1,0 & 12,0 & 87,0 \\
& 100 & 1,7 & 9,8 & 88,5 & 3,3 & 8,1 & 88,6 \\
& 200 & 0,0 & 0,0 & 100,0 & 4,4 & 5,8 & 89,8 \\
\hline
\end{tabular}




\subsection{Aplicação}

$\mathrm{Na}$ aplicação consideram-se dois conjuntos de dados reais. O primeiro conjunto de dados é referente aos números de Tribolium confusum adultos cultivados em $29^{\circ} \mathrm{C}$, que foi apresentado por Eugene et al. (2002). Ajustamos os modelos propostos e os submodelos correspondentes aos conjuntos de dados. Para efeito de comparação ajustamos também os modelos Kw-Normal (Cordeiro \& Castro, 2011) e Beta-Normal (Eugene et al., 2002). De acordo com os critérios AIC e SBC, os modelos WBN-PA e WG-PA mostram poucas diferenças entre si nos ajustes, e apresentam o melhor ajuste do que outros modelos considerados.

TABELA 2.7: AIC e SBC para os modelos ajustados

\begin{tabular}{lcccc}
\hline Modelo & Número dos parâmetros & $\ell(\hat{\boldsymbol{\xi}})$ & AIC & SBC \\
\hline WBN-PA & 4 & $-3566,1$ & 7140,2 & 7158,3 \\
WG-PA & 3 & $-3570,9$ & 7147,9 & 7161,5 \\
Beta normal & 4 & $-3584,5$ & 7176,9 & 7195,0 \\
Kw-normal & 4 & $-3584,7$ & 7177,4 & 7195,5 \\
WP-PA & 3 & $-3602,8$ & 7211,6 & 7225,1 \\
WBN-AA & 2 & $-3605,1$ & 7214,2 & 7223,3 \\
WG-UA & 3 & $-3605,1$ & 7216,1 & 7229,7 \\
WP-UA & 3 & $-3605,1$ & 7216,2 & 7229,8 \\
WBN-UA & 4 & $-3605,1$ & 7218,2 & 7236,3 \\
\hline
\end{tabular}

Os modelos ajustados sobrepostos ao histograma dos dados na Figura 2.6 reforçam os resultados da Tabela 2.5 para o modelo WBN-PA. O desvio médio absoluto (DMA) entre a frequência observada e esperada atingiu o valor mínimo para o modelo WBN-PA. As estimativas de máxima verossimilhança dos parâmetros do modelo WBN-PA são dadas por $\hat{\alpha}=6.1591$, $\hat{\lambda}=-33.7471, \hat{\eta}=2.7762$ e $\hat{\theta}=50.0055$.

Usando a estatística de RV para testar a hipótese nula $H_{0}$ : WG-PA , $H_{0}$ : WP-PA $H_{0}$ : WBN-AA contra a hipótese alternativa $H_{1}$ : WBN-PA são $9,698(p-$ valor $=0,021)$, 73, 390, $(p$-valor < 0,001) e 78, $020(p$-valor $<0,001)$, respectivamente, escolhendo o nível de significância usual (5\%), rejeitamos ambas hipóteses nulas em favor da distribuição WBN-PA. 
TABELA 2.8: Frequências observadas e esperadas dos números de adultos de Tribolium confusum cultivados em $29^{\circ} \mathrm{C}$ e DMA entre as frequências

\begin{tabular}{lccccc}
\hline & & \multicolumn{4}{c}{ Esperada } \\
\cline { 3 - 6 } Números de Adultos & Observada & $K w$-normal & Beta normal & GW-PA & WBN-PA \\
\hline 30 & 1 & 0,21 & 0,22 & 1,40 & 0,47 \\
50 & 1 & 5,77 & 5,67 & 10,31 & 6,49 \\
70 & 40 & 37,43 & 37,39 & 36,47 & 33,95 \\
90 & 96 & 91,85 & 93,70 & 82,18 & 92,02 \\
110 & 122 & 125,53 & 127,54 & 125,27 & 135,11 \\
130 & 140 & 123,95 & 123,73 & 133,97 & 127,28 \\
150 & 92 & 102,41 & 100,77 & 109,17 & 97,37 \\
170 & 70 & 75,95 & 74,30 & 75,33 & 69,42 \\
190 & 44 & 52,00 & 50,97 & 47,89 & 48,43 \\
210 & 38 & 33,18 & 32,80 & 29,42 & 33,22 \\
230 & 25 & 19,80 & 19,86 & 17,75 & 21,98 \\
250 & 13 & 11,06 & 11,32 & 10,44 & 13,46 \\
270 & 4 & 5,79 & 6,08 & 5,80 & 7,09 \\
290 & 1 & 2,84 & 3,08 & 2,90 & 2,86 \\
310 & 1 & 1,31 & 1,47 & 1,21 & 0,75 \\
330 & 2 & 0,56 & 0,66 & 0,38 & 0,10 \\
\hline Total & 690 & 689,6 & 689,5 & 689,9 & 690,0 \\
DMA & & 4,60 & 4,39 & 5,42 & 4,23 \\
\hline
\end{tabular}
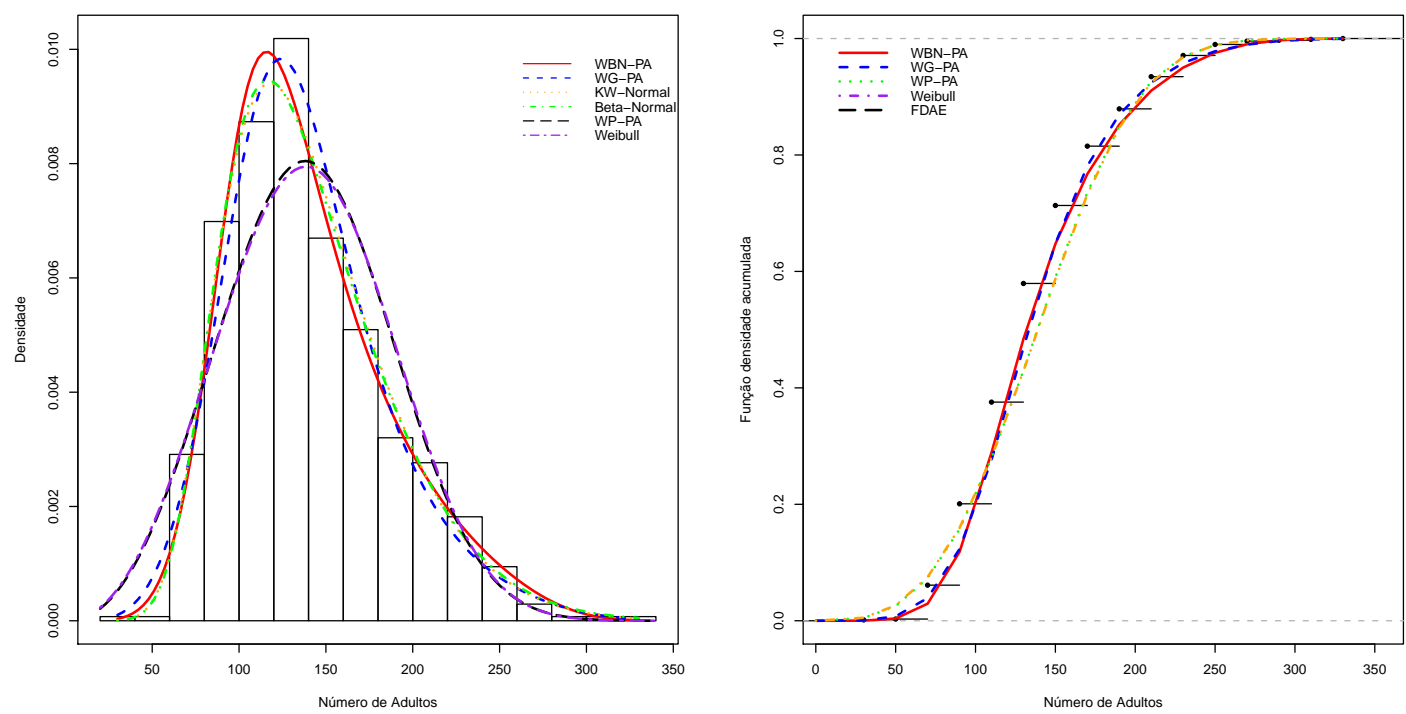

FIGURA 2.6: Figura da função densidade de probabilidade e função densidade acumulada dos modelos ajustados

O segundo conjunto de dados é obtido a partir de Smith \& Naylor (1987), que representam a força de ruptura de fibra de vidros com $1,5 \mathrm{~cm}$, os quais foram medidos no Laboratório Nacional de Física da Inglaterra; porém, infelizmente não temos as unidades de medida. O 
conjunto de dados é: $0,550,93$ 1,25 1,36 1,49 1,52 1,58 1,61 1,64 1,68 1,73 1,81 2,00 0,74 1,04 1,27

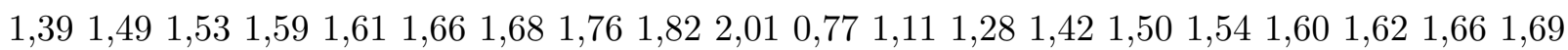

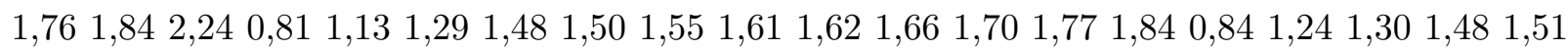

$1,551,61 \quad 1,631,67 \quad 1,701,781,89$.

Recentemente, este conjunto de dados foi ajustado ao modelo Beta Generalizado Exponencial introduzido por Barreto-Souza et al. (2010). Na Tabela 2.9 apresentamos as estimativas de função de verossimilhança, os números dos parâmetros e os valores de AIC dos modelos ajustados. De acordo com o critério AIC, o modelo WBN-UA é mais adequado do que outros modelos considerados.

TABELA 2.9: Estimativas de função de verossimilhança e valor AIC para as distribuições ajustadas.

\begin{tabular}{lcccc}
\hline Modelo & Número dos parâmetros & $\ell(\hat{\xi})$ & AIC & SBC \\
\hline WBN-UA & 4 & $-11,4$ & 30,8 & 39,3 \\
WG-UA & 3 & $-12,0$ & 30,1 & 36,5 \\
WP-UA & 3 & $-13,5$ & 32,9 & 39,4 \\
Weibull & 2 & $-15,2$ & 34,4 & 38,7 \\
WG-PA & 3 & $-15,2$ & 36,4 & 43,0 \\
WBN-PA & 4 & $-15,2$ & 38,4 & 47,0 \\
WP-PA & 3 & $-15,2$ & 36,4 & 42,9 \\
\hline
\end{tabular}

TABELA 2.10: As estimativas de máxima verossimilhança dos parâmetros para os modelos WBN-UA, WG-UA, WP-UA e Weibull

\begin{tabular}{lcccc}
\hline Modelo & $\alpha$ & $\lambda$ & $\eta$ & $\theta$ \\
\hline WBN-UA & 2,00 & 1,02 & 2,25 & 1336,9 \\
WG-UA & 3,20 & $-0,36$ & - & 15,64 \\
WP-UA & 4,48 & -1.64 & - & 2,38 \\
Weibull & 5,78 & $-2,82$ & - & - \\
\hline
\end{tabular}

Usando a estatística de RV para testar a hipótese nula $H_{0}$ : WG-UA, $H_{0}$ : WP-UA e $H_{0}$ : Weibull contra a hipótese alternativa $H_{1}:$ WBN-UA, temos $1,305(p-$ valor $=0,253), 4,187$ $(p-$ valor $=0,041)$ e 7,652 $(p-$ valor $=0,022)$, respectivamente. Assim, escolhendo o nível de significância usual (5\%), a distribuição WG-UA e WBN-UA não são significativamente diferentes, no entanto a distribuição WP-UA e Weibull são significativamente diferentes da WBN-UA. A Figura 2.7 mostra as $f d p$ e $f d a$ dos modelos ajustados sobrepostas no histograma e a função de distribuição empírica acumulada, reforçando o resultado da Tabela 2.9. 

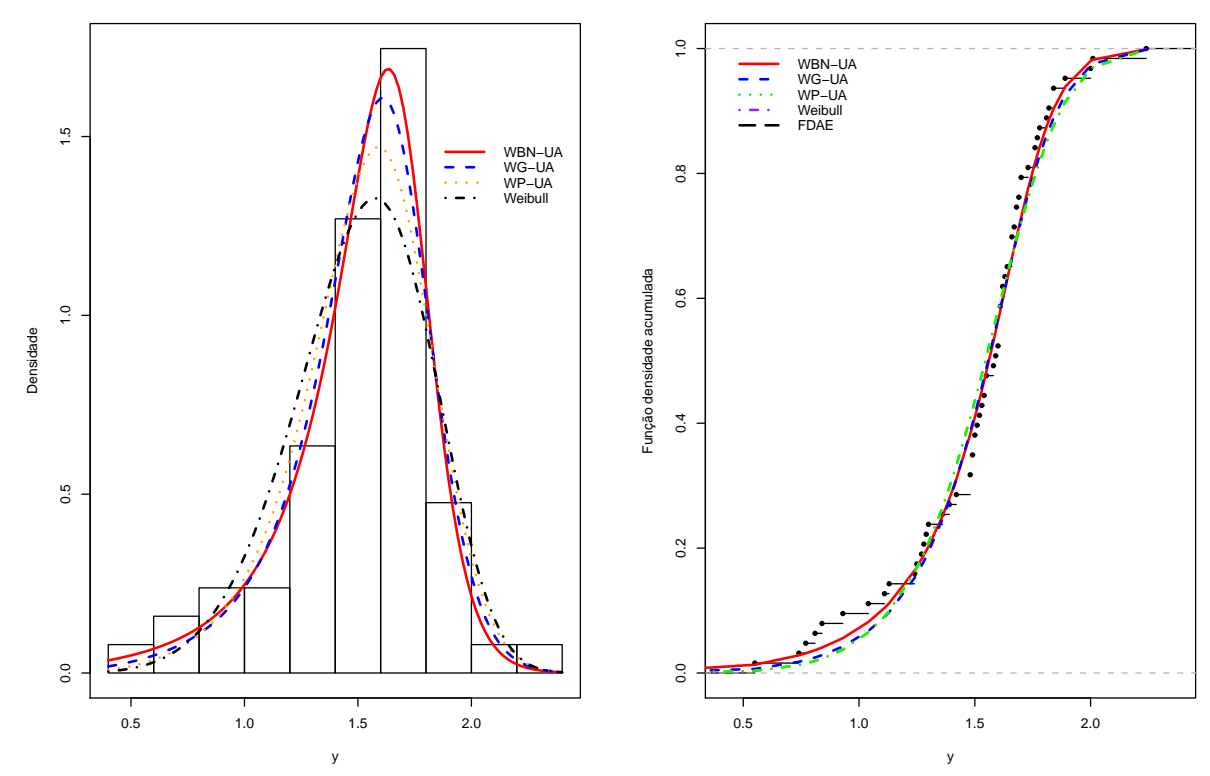

FIGURA 2.7: Figura da função densidade de probabilidade e função densidade acumulada dos quatro melhores modelos ajustados

\subsection{Conclusão}

Neste trabalho, apresentamos a distribuição WBN sob uma estrutura de ativação latente, que inclui vários tipos de distribuições para modelar dados de sobrevivência. A flexibilidade da distribuição WBN é facilmente observada quando consideramos a sua função de risco, a qual pode acomodar várias formas. Obtivemos várias propriedades matemáticas padrões dos modelos propostos e os momentos tem forma fechada para alguns casos especiais. Usamos o teste RV e os critérios AIC e BSC para comparar o modelo proposto com seus sub-modelos e algumas distribuições da literatura. Finalmente, os dois conjuntos de dados reais da literatura são ajustados ao modelo WBN para mostrar a flexibilidade da nova distribuição. 


\section{Capítulo 3}

\section{Modelo de regressão de \\ Log-Weibull-Binomial-Negativo}

\subsection{Introdução}

Em muitas aplicações práticas, os tempos de vida são afetados por vários fatores, os quais são referidos como covariáveis (ou variáveis explicativas), tais como o nível de colesterol, pressão arterial e muitos outros. Sendo assim, é importante explorar a relação entre o tempo de vida e as variáveis explicativas e uma maneira de estudar essa relação é por meio do modelo de regressão locação-escala, também conhecido como modelo de tempo de vida acelerado (Nelson, 1990). Neste capítulo propomos o modelo de regressão locação-escala baseado na distribuição WBN sob diferentes esquemas de ativação, denominado log-WBN (LWBN).

Após o ajuste do modelo, é importante verificar seus pressupostos e realizar estudos de sensibilidade para detectar as possíveis observações influentes ou extremas que podem causar distorções sobre os resultados da análise; porém, os métodos de diagnóstico tem sido uma ferramenta importante na análise de regressão. Cook (1977) propôs medidas de diagnóstico tais como deleção de casos e influência local, as quais tem sido amplamente aplicadas aos modelos de regressão.

Nesta capítulo discutimos a análise de influência caso a caso a partir de uma perspectiva Bayesiana, com o objetivo de detectar observações influentes que podem causar importantes distorções nos resultados da análise. A metodologia considerada neste estudo é baseada nas medidas de $\psi$-divergência (Peng \& Dey, 1995; Weiss, 1996). 


\subsection{Formulação do modelo de regressão LWBN}

Neste seção apresentamos os modelos de regressão Log-WBN (LWBN) sob diferentes esquemas de ativação.

\section{Modelo LWBN-PA}

Seja $Y$ uma variável aleatória não negativa da distribuição WBN-PA com $f d p$ dada em (2.15) e considerando a transformação $Z=\log (Y)$ e a reparametrização $\lambda=-\mu / \sigma$ e $\alpha=1 / \sigma$. Assim, a $f d p$ de $Z$ tem expressão como

$$
f(z ; \boldsymbol{\xi})=\frac{\frac{\theta}{\sigma} \exp \left[\frac{z-\mu}{\sigma}-\exp \left(\frac{z-\mu}{\sigma}\right)\right]\left(1+\eta \theta\left[1-\exp \left(1-\exp \left(\frac{z-\mu}{\sigma}\right)\right)\right]\right)^{-(1 / \eta+1)}}{1-(1+\eta \theta)^{-1 / \eta}}, z \in \mathbb{R}
$$

em que $\boldsymbol{\xi}=(\eta, \theta, \sigma, \mu), \eta>-1 / \theta, \theta>0, \sigma>0$ e $\mu \in \mathbb{R}$. A Figura 3.1(a) mostra algumas formas da $f d a$ da variável aleatória $Z$ que é chamada distribuição Log-WBN-PA (LWBN-PA).

As funções de sobrevivência e risco correspondente são dadas por

$$
S(z ; \boldsymbol{\xi})=\frac{\left(1+\theta \eta\left[1-\exp \left\{-\exp \left(\frac{z-\mu}{\sigma}\right)\right\}\right]\right)^{-1 / \eta}-(1+\eta \theta)^{-1 / \eta}}{1-(1+\eta \theta)^{-1 / \eta}}, \quad z \in \mathbb{R}
$$

e

$$
h(z ; \boldsymbol{\xi})=\frac{\frac{\theta}{\sigma} \exp \left[\frac{z-\mu}{\sigma}-\exp \left(\frac{z-\mu}{\sigma}\right)\right]\left(1+\eta \theta\left[1-\exp \left(1-\exp \left(\frac{z-\mu}{\sigma}\right)\right)\right]\right)^{-(1 / \eta+1)}}{\left(1+\theta \eta\left[1-\exp \left\{-\exp \left(\frac{z-\mu}{\sigma}\right)\right\}\right]\right)^{-1 / \eta}-(1+\eta \theta)^{-1 / \eta}}, z \in \mathbb{R}
$$

respectivamente. Note que a fpd dada em (3.1) também pode ser escrita como

$$
f(y ; \xi)=\sum_{m=1}^{\infty} \frac{p m}{\log m} f(z \mid m, \mu, \sigma)
$$

no qual $f(z \mid m, \alpha, \lambda)=\frac{m}{\sigma} \log (m) \exp \left\{\frac{z-\mu}{\sigma}-m \exp \left(\frac{z-\mu}{\sigma}\right)\right\}$ tem a distribuição Valor Extremo com parâmetro de escala $\sigma / \log (m)$, parâmetro de forma $\mu$, e $p_{m}$ dado em (2.3). A função de risco da distribuição LWBN-PA é apresentada na Figura 3.1(b) para alguns parâmetros selecionados. 
(a)

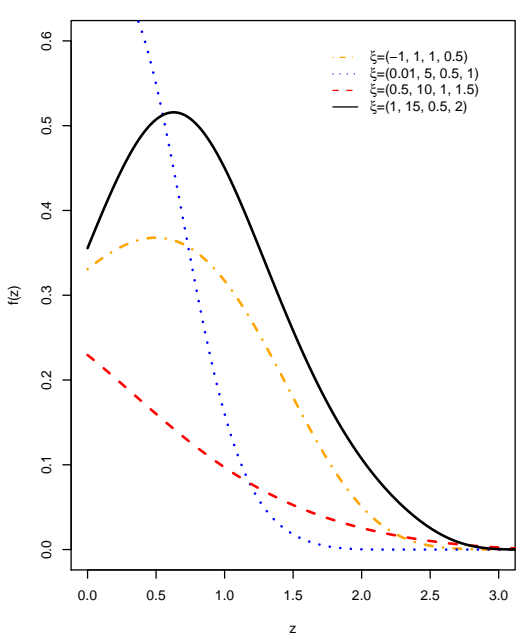

(b)

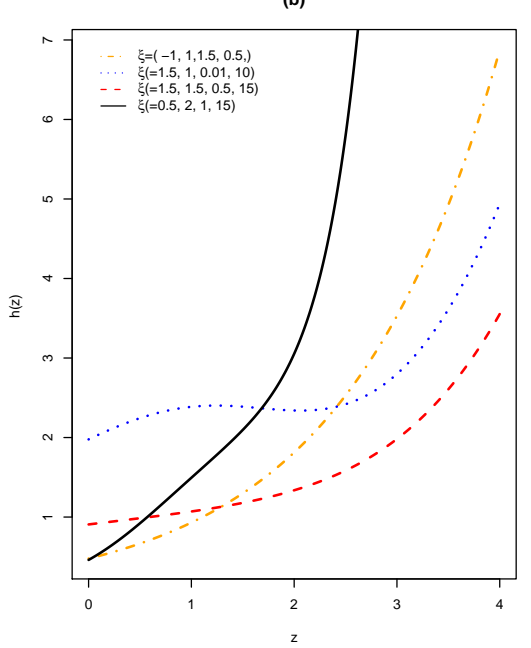

FIGURA 3.1: Funções de (a) densidade probabilidade e (b) risco da distribuição LWBN-PA para alguns parâmetros selecionados

\section{Função geradora de momentos}

A $(f g m) M(t)=E[\exp (t Z)]$ utiliza-se da expansão em série de potência para a função exponencial e da (3.2) para obter

$$
M(t)=\exp (\mu t) \sum_{m=1}^{\infty} \frac{p_{m}}{\log (m)} \Gamma\left(1+\frac{\sigma t}{\log (m)}\right), t>-\log (m) / \sigma
$$

Logo, o k-ésimo momento ordinário de $Z$ pode ser obtido por $E\left[Y^{k}\right]=M^{(k)}(t)=\frac{d^{k} M}{d t^{k}}(0)$.

\section{Modelo LWBN-UA}

Analogamente, a $f d p$ de $Z$ em que $Y$ tem a distribuição WBN-UA dada em (2.28) tem expressão como

$$
f(z ; \boldsymbol{\xi})=\frac{\frac{\theta}{\sigma}\left[\exp \left\{\frac{z-\mu}{\sigma}-\exp \left(\frac{z-\mu}{\sigma}\right)\right\}\right]\left(1+\eta \theta\left[\exp \left\{-\exp \left(\frac{z-\mu}{\sigma}\right)\right\}\right]\right)^{-(1 / \eta+1)}}{1-(1+\eta \theta)^{-1 / \eta}}, z \in \mathbb{R}
$$

em que $\boldsymbol{\xi}=(\eta, \theta, \sigma, \mu), \eta>-1 / \theta, \theta>0, \sigma>0$ e $\mu \in \mathbb{R}$. A Figura 3.2(a) mostra algumas formas da $f d a$ da variável aleatória $Z$ que é chamada distribuição Log-WBN-UA (LWBN-UA).

As funções de sobrevivência e risco correspondentes são dadas por

$$
S(z ; \boldsymbol{\xi})=\frac{1-\left(1+\eta \theta\left[\exp \left\{-\exp \left(\frac{z-\mu}{\sigma}\right)\right\}\right]\right)^{-1 / \eta}}{1-(1+\eta \theta)^{-1 / \eta}}, z \in \mathbb{R}
$$


$\mathrm{e}$

$$
h(z ; \boldsymbol{\xi})=\frac{\frac{\theta}{\sigma}\left[\exp \left\{\frac{z-\mu}{\sigma}-\exp \left(\frac{z-\mu}{\sigma}\right)\right\}\right]\left(1+\eta \theta\left[\exp \left\{-\exp \left(\frac{z-\mu}{\sigma}\right)\right\}\right]\right)^{-(1 / \eta+1)}}{1-\left(1+\eta \theta\left[\exp \left\{-\exp \left(\frac{z-\mu}{\sigma}\right)\right\}\right]\right)^{-1 / \eta}}, z \in \mathbb{R}
$$

respectivamente. Note que a $f d p$ dada em (3.3) também pode ser escrita como

$$
f(y ; \xi)=\sum_{m=1}^{\infty} \sum_{k=0}^{m-1}(-1)^{k}\left(\begin{array}{c}
m-1 \\
k
\end{array}\right) \frac{m p_{m}}{(k+1) \log (k+1)} f(z \mid m, \mu, \sigma),
$$

em que $f(z \mid m, \alpha, \lambda)=\frac{(k+1) \log (k+1)}{\sigma} \exp \left\{\frac{z-\mu}{\sigma}-(k+1) \exp \left(\frac{z-\mu}{\sigma}\right)\right\}$ tem a distribuição Valor Extremo com parâmetro de escala $\sigma / \log (k+1)$, parâmetro de forma $\mu$, e $p_{m}$ dada em $(2.3)$. A função de risco da distribuição LWBN-UA é apresentada na Figura 3.2(b) para alguns parâmetros selecionados.
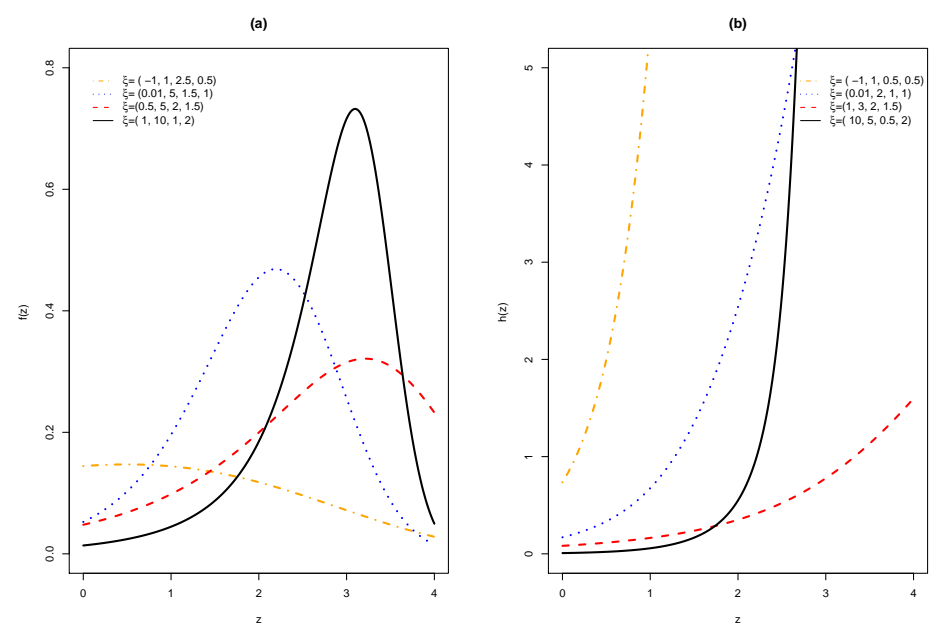

FIGURA 3.2: Funções de (a) densidade probabilidade e (b) risco da distribuição LWBN-UA para alguns parâmetros selecionados

\section{Funções geradora de momentos}

A $(f g m) M(t)=E[\exp (t Z)]$ utiliza-se da expansão em série de potências para a função exponencial e da (2.29) para obter

$$
M(t)=\sum_{m=1}^{\infty} \sum_{k=0}^{m-1}(-1)^{k}\left(\begin{array}{c}
m-1 \\
k
\end{array}\right) \frac{m p_{m} \exp (\mu t)}{(k+1) \log (k+1)} \Gamma\left(1+\frac{\sigma t}{\log (k+1)}\right), \quad t>-\log (k+1) / \sigma
$$

Logo, o k-ésimo momento ordinário de $Z$ pode ser obtido por $E\left[Y^{k}\right]=M^{(k)}(t)=\frac{d^{k} M}{d t^{k}}(0)$. 
Os modelos (3.1) e (3.3) podem ser escritos como o modelo log-linear (ou locação-escala)

$$
Z=\mu+\sigma W
$$

em que a função densidade da variável $W$ sobre esquema de primeira ativação é dada por

$$
f_{\mathrm{p}}(w)=\frac{\frac{\theta}{\sigma} \exp [w-\exp (w)](1+\eta \theta[1-\exp (1-\exp (w))])^{-(1 / \eta+1)}}{1-(1+\eta \theta)^{-1 / \eta}} w \in \mathbb{R}
$$

e a função densidade da variável $W$ sobre última ativação é dada por

$$
f_{\mathrm{u}}(w)=\frac{\frac{\theta}{\sigma}[\exp \{w-\exp (w)\}](1+\eta \theta[\exp \{-\exp (w)\}])^{-(1 / \eta+1)}}{1-(1+\eta \theta)^{-1 / \eta}}, w \in \mathbb{R}
$$

em que $\eta>-1 / \theta, \theta>0, \sigma>0$ e $\mu \in \mathbb{R}$. A transformação $Z=\log (Y)$ pertence à família de locação-escala, em que $\sigma$ é o parâmetro de escalar e o parâmetro de locação $\mu$, que depende do vetor de covariáveis $\mathbf{x}^{\top}=\left(x_{1}, \cdots, x_{p}\right)$, denotado por $\mu_{i}=\mathbf{x}^{\top} \beta$, em que $\boldsymbol{\beta}=\left(\beta_{0}, \cdots, \beta_{p}\right)^{\top}$, é o vetor dos parâmetros a serem estimados. O modelo de regressão baseado na distribuição LWBN sob esquemas de primeira ativação e última ativação dadas em (3.1) e (3.3) respectivamente é dado por

$$
Z=\mathbf{x}^{\top} \boldsymbol{\beta}+\sigma W
$$

em que $W$ tem $f d p$ dada em (3.6) ou (3.7). No caso da distribuição LWBN sob esquema de ativação aleatória $W$ tem a distribuição Valor Extremo padrão (Lawless, 2003). Nesse caso, a fdp de $Z$ dado $\mathrm{x}$ é dada por

$$
f(Z \mid \mathbf{x})=\frac{1}{\sigma} \exp \left\{\frac{Z-\mathbf{x}^{\top} \beta}{\sigma}-\exp \left(\frac{Z-\mathbf{x}^{\top} \beta}{\sigma}\right)\right\} .
$$

A função de sobrevivência correspondente é dada por

$$
S(Z \mid \mathbf{x})=\exp \left\{-\exp \left(\frac{Z-\mathbf{x}^{\top} \beta}{\sigma}\right)\right\}
$$

A $f d p$ de $Z$ dado $\mathbf{x}$ sob esquema de primeira ativação é dada por

$$
f(Z \mid \mathbf{x})=\frac{\frac{\theta}{\sigma} \exp \left[\frac{Z-\mathbf{x}^{\top} \beta}{\sigma}-\exp \left(\frac{Z-\mathbf{x}^{\top} \beta}{\sigma}\right)\right]\left(1+\eta \theta\left[1-\exp \left(1-\exp \left(\frac{Z-\mathbf{x}^{\top} \beta}{\sigma}\right)\right)\right]\right)}{1-(1+\eta \theta)^{-1 / \eta}}
$$


e a função de sobrevivência correspondente é dada por

$$
S(Z \mid \mathbf{x})=\frac{\left(1+\theta \eta\left[1-\exp \left\{-\exp \left(\frac{Z-\mathbf{x}^{\top} \beta}{\sigma}\right)\right\}\right]\right)^{-1 / \eta}-(1+\eta \theta)^{-1 / \eta}}{1-(1+\eta \theta)^{-1 / \eta}} .
$$

A $f d p$ de $Z$ dado $\mathbf{x}$ sob esquema de última ativação é dada por

$$
f(Z \mid \mathbf{x})=\frac{\frac{\theta}{\sigma}\left[\exp \left\{\frac{Z-\mathbf{x}^{\top} \beta}{\sigma}-\exp \left(\frac{Z-\mathbf{x}^{\top} \beta}{\sigma}\right)\right\}\right]\left(1+\eta \theta\left[\exp \left\{-\exp \left(\frac{Z-\mathbf{x}^{\top} \beta}{\sigma}\right)\right\}\right]\right)^{-(1 / \eta+1)}}{1-(1+\eta \theta)^{-1 / \eta}},
$$

e a função de sobrevivência correspondente é dada por

$$
S(Z \mid \mathbf{x})=\frac{1-\left(1+\eta \theta\left[\exp \left\{-\exp \left(\frac{Z-\mathbf{x}^{\top} \beta}{\sigma}\right)\right\}\right]\right)^{-1 / \eta}}{1-(1+\eta \theta)^{-1 / \eta}}
$$

\subsection{Inferência Bayesiana}

Considerando a situação em que o tempo de vida $Y$ dado na seção 3.2 não é observado e está sujeito à censura à direita. Seja $C_{i}$ é o tempo de censura. Em uma amostra de tamanho $n$, observamos $t_{i}=\min \left\{\log \left(Y_{i}\right), C_{i}\right\}$ e $\delta_{i}=I\left(\log \left(Y_{i}\right) \leq \log \left(C_{i}\right)\right)$ tais que $\delta_{i}=1$ se $t_{i}$ é o logaritmo do tempo de vida e $\delta_{i}=0$ é o caso contrário. As funções de verossimilhança dos modelos são dadas em (3.8), (3.9), e (3.10) para o vetor dos parâmetros $\boldsymbol{\vartheta}=\left(\boldsymbol{\xi}^{\top}, \sigma, \boldsymbol{\beta}^{\top}\right)^{\top}$. Em condições censura à direita e não-informativa, a função é dada por

$$
L(\boldsymbol{\vartheta} ; \mathcal{D}) \propto \prod_{i=1}^{n} f\left(t_{i} ; \boldsymbol{\vartheta}\right)^{\delta_{i}} S\left(t_{i} ; \boldsymbol{\vartheta}\right)^{1-\delta_{i}}
$$

em que $\boldsymbol{\xi}=(\eta, \theta)$ é um vetor dos parâmetros dos modelos dado em (3.9) e (3.10), $\mathcal{D}=(\boldsymbol{t}, \boldsymbol{\delta}, \boldsymbol{x})$, $\boldsymbol{t}=\left(t_{1}, \ldots, t_{n}\right)^{\top}, \boldsymbol{x}=\left(\boldsymbol{x}_{1}, \ldots, \boldsymbol{x}_{n}\right)^{\top}$ e $\boldsymbol{\delta}=\left(\delta_{1}, \ldots, \delta_{n}\right)^{\top}$, em que $f(\cdot ; \boldsymbol{\vartheta})$ e $S(\cdot ; \boldsymbol{\vartheta})$ são as funções de densidade e sobrevivência dadas na seção 3.2 .

Assumindo as seguintes densidades a priori para os parâmetros $\sigma, \eta, \theta, \boldsymbol{\beta}^{\top}$,

- $\sigma \sim N\left(\mu_{\sigma}, \sigma_{\sigma}^{2}\right) I(\sigma>0), \mu_{\sigma}$ e $\sigma_{\sigma}$ conhecidos;

- $\eta, \theta \sim N\left(\mu_{\eta}, \sigma_{\eta}^{2}\right) I(\eta>-1 / \theta) N\left(\mu_{\theta}, \sigma_{\theta}^{2}\right) I(\theta>0), \mu_{\eta}, \sigma_{\eta}, \mu_{\theta}$ e $\sigma_{\theta}$ conhecidos;

- $\beta_{j} \sim N\left(\mu_{0 j}, \sigma_{0 j}^{2}\right), \mu_{0 j}$ e $\sigma_{0 j}$ conhecidos, $j=0, \cdots, p$,

em que $N\left(\mu, \sigma^{2}\right) I(x>a)$ denote a distribuição normal trucada no intervalo $(a, \infty)$ com $a \in \mathbb{R}$. A 
escolha da distribuição de probabilidade Normal para as prioris, facilita a inserção de informação em uma determinada região de interesse através dos parâmetros $\mu$ e $\sigma^{2}$ da distribuição Normal $N\left(\mu, \sigma^{2}\right)$, mesmo que no caso de truncamento esses hiperparâmetros não representem a média e a variância, mas ainda controlam a região de maior massa de probabilidade. Assumindo que $\sigma \mathrm{e}$ $\beta_{j}$ são parâmetros independentes, e que os parâmetros $\eta$ e $\theta$ são independentes entre si, a função densidade a priori para $\boldsymbol{\vartheta}$ pode ser escrita como

$$
\pi(\boldsymbol{\vartheta})=\prod_{j=1}^{p} \pi\left(\beta_{j}\right) \pi(\sigma) \pi(\eta, \theta) .
$$

Para que as densidades a priori possuem informação vaga, consideramos que $\mu_{\sigma}=\mu_{\eta}=\mu_{0 j}=0$ com $\sigma_{\sigma}^{2}, \sigma_{\eta}^{2}, \sigma_{\theta}^{2}$ e $\sigma_{0 j}^{2}$ assumem valores grandes.

Combinando a função de verossimilhança (3.11) e as distribuições a priori dadas em (3.12), a densidade conjunta a posteriori para $\boldsymbol{\vartheta}$ é obtida como

$$
\pi(\boldsymbol{\vartheta} \mid \mathcal{D}) \propto L(\boldsymbol{\vartheta} ; \mathcal{D}) \prod_{j=1}^{p} \pi\left(\beta_{j}\right) \pi(\sigma) \pi(\eta, \theta)
$$

Como a densidade conjunta a posteriori é difícil de ser tratada analiticamente, uma possível solução é utilizar os métodos de Monte Carlo via Cadeias de Markov (MCMC), tais como o Amostrador de Gibbs e o Metropolis-Hasting. Esses algoritmos podem ser utilizados para gerar amostras da distribuição a posteriori dos parâmetros $\pi(\boldsymbol{\vartheta} \mid \mathcal{D})$ por meio das distribuições condicionais completas $\pi\left(\vartheta_{i} \mid \boldsymbol{\vartheta}_{-i}, \mathcal{D}\right)$, sendo $\boldsymbol{\vartheta}_{-i}$ o vetor de todos os parâmetros exceto o parâmetro $\vartheta_{i}$. O algoritmo amostrador de Gibbs (Gamerman \& Lopes, 2006) pode ser utilizado quando as distribuições condicionais completas tem formas conhecidas, caso contrário, deve-se utilizar o algoritmo Metropolis-Hastings.

Como as distribuições condicionais completas dos modelos propostos não são identificáveis, o algoritmo Metropolis-Hastings foi utilizado nesse trabalho. Na literatura, é conhecida que a simulação MCMC na reta é mais eficiente, por isso utilizaremos a transformação paramétrica $\boldsymbol{\varphi}=(\log (\eta), \log (\theta), \log (\sigma), \boldsymbol{\beta})=\left(\varphi_{1}, \varphi_{2}, \varphi_{3}, \boldsymbol{\beta}\right)$. Dessa forma, obtemos o espaço do paramétrico $\mathbb{R}^{p+3}$ sendo que a densidade conjunta a posteriori é dada por

$$
\begin{gathered}
\pi(\boldsymbol{\varphi} ; \mathcal{D}) \propto \quad L(\boldsymbol{\varphi} ; \mathcal{D}) \exp \left\{-\frac{1}{2}\left[\sum_{j=1}^{p}\left(\frac{\beta_{0 j}^{2}}{\sigma_{0 j}}\right)^{2}+\frac{2 \exp \left(\varphi_{1}\right)}{\sigma_{\eta}{ }^{2}}+\left(\frac{\exp \left(\varphi_{2}\right)-\mu_{\theta}}{\sigma_{\theta}}\right)^{2}\right.\right. \\
\left.\left.+\frac{2 \exp \left(\varphi_{3}\right)}{\sigma_{\sigma}{ }^{2}}+\varphi_{1}+\varphi_{2}+\varphi_{3}\right]\right\}\left(1-\Phi\left(-e^{-\varphi_{2}}\right)\right)^{-1}
\end{gathered}
$$


O algoritmo Metropolis-Hastings é dado por:

(1) Iniciar com um valor $\varphi_{(0)}$, e o indicador de estágio $j=0$;

(2) Gerar um ponto $\varphi^{\prime}$ do núcleo de transição $Q\left(\varphi^{\prime}, \varphi_{j}\right)$;

(3) Atualizar $\varphi_{(j)}$ para $\varphi_{(j+1)}=\varphi^{\prime}$ com probabilidade

$$
p=\min \left\{1, \frac{\pi\left(\varphi^{\prime} \mid \mathcal{D}\right) Q\left(\varphi_{j}, \varphi^{\prime}\right)}{\pi\left(\boldsymbol{\varphi}_{(j)} \mid \mathcal{D}\right) Q\left(\left(\boldsymbol{\varphi}^{\prime}, \boldsymbol{\varphi}_{j}\right)\right.}\right\}
$$

e manter $\varphi_{(j)}$ com probabilidade $1-p$;

(4) Repetir os estágios (2) e (3) até conseguir uma distribuição estacionária.

Nesse trabalho consideramos o núcleo de transição $Q\left(\varphi^{\prime}, \varphi_{j}\right)=N_{p+3}\left(\varphi_{j}, \tilde{\Sigma}_{\varphi}\right)$, em que $\tilde{\Sigma}_{\varphi}$ é uma matriz de covariância, que é a mesma para todos estágios, assim a probabilidade de movimento se reduz à $p_{=} \min \left\{1, \pi\left(\varphi^{\prime} \mid \mathcal{D}\right) / \pi\left(\boldsymbol{\varphi}_{(j)} \mid \mathcal{D}\right)\right\}$. Apesar da escolha de $\tilde{\Sigma}_{\varphi}$ ser livre, a convergência do algoritmo será mais rápida se utilizamos a matriz de covariância dos parâmetros, portanto, uma estimativa para $\tilde{\Sigma}_{\varphi}$ consiste no inverso da matriz de Informação de Fisher.

\subsection{Estudo de dados artificiais}

Um estudo de simulação foi realizado com objetivo de comparar o modelo de regressão proposto LWBN na presença dos esquemas de primeira ativação (LWBN-PA), última ativação (LWBN-UA) e ativação aleatória (LWBN-AA).

Os critérios DIC, EAIC, EBIC, LPML foram considerados para a comparação de modelo e as estimativas da função de sobrevivência na mediana para cada nível da covariável, os respectivos erros padrão (EP) e as raízes quadradas do erro quadrático médio(RQEM) foram calculados utilizando as médias a posteriori dos parâmetros dos modelos ajustados.

Na simulação, consideramos a covariável binária $\mathbf{x}$, que foi gerada pela distribuição Bernoulli com parâmetros 0,5 , e fixamos os valores dos parâmetros $\beta_{0}=5,0 ; \beta_{1}=-0,5 ; \eta=3$; $\theta=10$ e $\sigma=0,2$. Para cada modelo proposto foi gerado uma amostra de tamanho 600, cujo tempo de vida foram gerados das distribuição proposta (através do método inverso) com os parâmetros escolhidos. Assim, os três modelos propostos foram ajustados para cada conjunto de dados gerados. 
As densidades a priori consideradas para a simulação são dadas na Seção 3.3 , com $\mu_{\eta}=10, \mu_{\sigma}=\mu_{\eta}=\mu_{0 j}=0, \sigma_{\sigma}^{2}=20^{2}, \sigma_{\eta}^{2}=20^{2}, \sigma_{\theta}^{2}=10^{2}$ e $\sigma_{0 j}^{2}=20^{2}$, e dessa forma as densidades a priori possuem a informação vaga. Para cada conjunto de dados foi gerada uma cadeia de tamanho 35.000 e as primeiras 17.500 iterações foram desconsideradas para eliminar o efeito dos valores iniciais, assim obtivemos uma amostra efetiva de tamanho 17.500 sobre a qual a posteriori é baseada.

A Tabela 3.1 apresenta as estimativas dos critérios Bayesianos EAIC, EBIC, DIC e LPML para a seleção dos modelos. Note que, de acordo com esses critérios, para cada conjunto de dados simulados, o modelo correspondente apresentou o melhor ajuste. Portanto, é possível discriminar os três modelos propostos para um conjunto de dados. Além disso, as estimativas da função de sobrevivência na mediana estão mais próximas do valor nominal $(0,5)$ e os respectivos RQEM são sempre menores para o melhor modelo ajustado, o que pode ser observado na Tabela 3.4 .

TABELA 3.1: Estimativas dos critérios Bayesianos dos modelos ajustados para os dados artificiais

\begin{tabular}{llcccc}
\hline \multirow{2}{*}{ Modelo Gerado } & Modelo Ajustado & \multicolumn{4}{c}{ Critério } \\
\cline { 3 - 6 } & & DIC & EAIC & EBIC & LPML \\
\hline \multirow{2}{*}{ LWBN-PA } & LWBN-PA & 476,5004 & 484,5609 & 506,5456 & $-239,1799$ \\
& LWBN-UA & 525,8179 & 535,8806 & 557,8652 & $-264,4000$ \\
& LWBN-AA & 526,5438 & 529,5035 & 542,6943 & $-263,2291$ \\
\hline LWBN-UA & LWBN-PA & $-552,9179$ & $-543,1026$ & $-521,118$ & 275,0185 \\
& LWBN-UA & $-575,0591$ & $-557,6462$ & $-536,6616$ & 281,5897 \\
& LWBN-AA & $-552,5393$ & $-549,4593$ & $-536,2686$ & 276,2594 \\
\hline LWBN-AA & LWBN-PA & $-45,0730$ & $-29,6031$ & $-7,6184$ & 17,9563 \\
& LWBN-UA & $-43,9017$ & $-27,7461$ & $-5,7614$ & 17,2582 \\
& LWBN-AA & $-37,2117$ & $-34,2013$ & $-21,0105$ & 18,6886 \\
\hline
\end{tabular}

TABELA 3.2: Estimativas da função de sobrevivência na mediana, EP e RQEM para os dados artificiais

\begin{tabular}{llcccccc}
\hline \multirow{2}{*}{ Modelo Gerado } & \multicolumn{3}{c}{$x=0$} & \multicolumn{3}{c}{$x=1$} \\
\cline { 3 - 8 } LWBN-PA & Modelo Ajustado & $\hat{S}_{\left(y_{\text {med }}\right)}$ & EP & RQEM & $\hat{S}_{\left(y_{\text {med }}\right)}$ & EP & RQEM \\
& LWBN-PA & 0,485 & 0,0203 & 0,0250 & 0,496 & 0,0209 & 0,0197 \\
& LWBN-UA & 0,534 & 0,0216 & 0,0402 & 0,511 & 0,0222 & 0,0250 \\
& LWBN-AA & 0,533 & 0,0216 & 0,0398 & 0,511 & 0,0219 & 0,0243 \\
\hline LWBN-UA & LWBN-PA & 0,470 & 0,0218 & 0,0375 & 0,473 & 0,0220 & 0,0350 \\
& LWBN-UA & 0,507 & 0,0217 & 0,0237 & 0,507 & 0,0213 & 0,0242 \\
& LWBN-AA & 0,472 & 0,0225 & 0,0360 & 0,476 & 0,0218 & 0,0324 \\
\hline LWBN-AA & LWBN-PA & 0,481 & 0,0217 & 0,0288 & 0,481 & 0,0249 & 0,0315 \\
& LWBN-UA & 0,492 & 0,0211 & 0,0219 & 0,492 & 0,0238 & 0,0246 \\
& LWBN-AA & 0,499 & 0,0204 & 0,0212 & 0,496 & 0,0232 & 0,0241 \\
\hline
\end{tabular}




\subsubsection{Análise de influência}

Um dos principais objetivos desse estudo é mostrar a necessidade dos modelos robustos de lidar com a presença da observação influente. Dois conjuntos de dados artificiais foram gerados a partir das distribuições LWBN-PA e LWBN-UA, baseada na mesma configuração da seção 3.4 .

Selecionamos os casos 45 e 350 para perturbar a fim de criarmos observações influentes no conjunto de dados; assim, escolhemos um ou dois casos e perturbamos a variável resposta da seguinte maneira $\widetilde{y}_{i}=y_{i}+4 S_{y}$ para $\mathrm{i}=45$ e 350 , em que $S_{y}$ são os desvios padrão dos tempos $z_{i}$. Desse modo, consideramos as seguinte configurações para o estudo:

- Configuração A: o conjunto de dados sem perturbação;

- Configuração B: dados com o caso 45 perturbado;

- Configuração C: dados com o caso 350 perturbado;

- Configuração D: dados com os casos 45 e 350 perturbados.

O algoritmo MCMC é o mesmo da última seção, e a convergência do algoritmo M-H é monitorada pelo diagnóstico de convergência Geweke proposto por Geweke (1992). A Tabela 3.3 apresenta as estimativas da função de sobrevivência na mediana para cada nível da covariável, os respectivos erros padrão (EP) e as raízes quadradas do erro quadrático médio(RQEM), que foram obtidos pelas médias a posteriori dos parâmetros dos modelos ajustados, para os conjuntos de dados gerados. Embora as distribuições a posteriori sejam pouco sensíveis à perturbação dos casos selecionados, de fato as estimativas da função de sobrevivência na mediana para cada covariável são próximas do valor teórico $(0,5)$, mas os respectivos EP e RQEM são maiores. A Tabela 3.4 apresenta as estimativas dos critérios Bayesianos EAIC, EBIC, DIC e LPML para cada configuração considerada, onde observamos que o modelo para os dados sem a perturbação apresenta um melhor ajuste. 
TABELA 3.3: Estimativas da função de sobrevivência na mediana, EP e RQEM para os dados artificiais

\begin{tabular}{|c|c|c|c|c|c|c|c|c|}
\hline \multicolumn{9}{|l|}{$\begin{array}{l}\text { Modelo Gerado } \\
\text { LWBN-PA }\end{array}$} \\
\hline \multirow{2}{*}{$\begin{array}{l}\text { Modelo } \\
\text { Ajustado }\end{array}$} & \multirow[b]{2}{*}{ Configuração } & \multirow{2}{*}{$\begin{array}{l}\text { Casos } \\
\text { Perturbados }\end{array}$} & \multicolumn{3}{|c|}{$x=0$} & \multicolumn{3}{|c|}{$x=1$} \\
\hline & & & $\left.\hat{S}_{(} y_{m e d}\right)$ & $\mathrm{EP}$ & RQEM & $\hat{S}_{\left(y_{m e d}\right)}$ & $\mathrm{EP}$ & RQEM \\
\hline \multirow[t]{4}{*}{ LWBN-PA } & A & nenhum & 0,485 & 0,0203 & 0,0250 & 0,496 & 0,0209 & 0,0197 \\
\hline & B & 45 & 0,471 & 0,0245 & 0,0377 & 0,537 & 0,0227 & 0,0433 \\
\hline & $\mathrm{C}$ & 350 & 0,512 & 0,0241 & 0,0268 & 0,540 & 0,0227 & 0,0459 \\
\hline & $\mathrm{D}$ & $\{45,350\}$ & 0,510 & 0,0250 & 0,0269 & 0,489 & 0,0236 & 0,0259 \\
\hline \multirow[t]{4}{*}{ LWBN-UA } & $\mathrm{A}$ & nenhum & 0,534 & 0,0216 & 0,0402 & 0,511 & 0,0222 & 0,0250 \\
\hline & B & 45 & 0,454 & 0,0248 & 0,0525 & 0,525 & 0,0242 & 0,0349 \\
\hline & $\mathrm{C}$ & 350 & 0,484 & 0,0260 & 0,0306 & 0,517 & 0,0232 & 0,0285 \\
\hline & $\mathrm{D}$ & $\{45,350\}$ & 0,507 & 0,0257 & 0,0266 & 0,492 & 0,0243 & 0,0256 \\
\hline \multicolumn{9}{|l|}{$\begin{array}{l}\text { Modelo Gerado } \\
\text { LWBN-PA }\end{array}$} \\
\hline \multirow{4}{*}{ LWBN-PA } & $\mathrm{A}$ & nenhum & 0,470 & 0,0218 & 0,0375 & 0,473 & 0,0220 & 0,0350 \\
\hline & B & 45 & 0,483 & 0,0221 & 0,0276 & 0,489 & 0,0219 & 0,0243 \\
\hline & $\mathrm{C}$ & 350 & 0,430 & 0,0220 & 0,0734 & 0,437 & 0,0217 & 0,0665 \\
\hline & $\mathrm{D}$ & $\{45,350\}$ & 0,435 & 0,0227 & 0,0689 & 0,439 & 0,0223 & 0,0647 \\
\hline \multirow[t]{4}{*}{ LWBN-UA } & A & nenhum & 0,507 & 0,0217 & 0,0237 & 0,507 & 0,0213 & 0,0242 \\
\hline & B & 45 & 0,521 & 0,0232 & 0,0315 & 0,516 & 0,0228 & 0,0276 \\
\hline & $\mathrm{C}$ & 350 & 0,477 & 0,0222 & 0,0316 & 0,478 & 0,0219 & 0,0310 \\
\hline & $\mathrm{D}$ & $\{45,350\}$ & 0,472 & 0,0235 & 0,0368 & 0,475 & 0,0221 & 0,0333 \\
\hline
\end{tabular}

TABELA 3.4: Estimativas dos critérios Bayesianos dos modelos ajustados para os dados artificiais

\begin{tabular}{|c|c|c|c|c|c|c|c|c|c|}
\hline \multirow{2}{*}{$\begin{array}{l}\text { Modelo } \\
\text { Gerado }\end{array}$} & \multirow[b]{2}{*}{ Configuração } & \multicolumn{4}{|c|}{ LWBN-PA } & \multicolumn{4}{|c|}{ LWBN-UA } \\
\hline & & DIC & EAIC & EBIC & LPML & DIC & EAIC & EBIC & LPML \\
\hline \multirow[t]{4}{*}{ LWBN-PA } & $\mathrm{A}$ & 476,5 & 484,6 & 506,5 & $-239,2$ & 525,8 & 535,9 & 557,9 & $-264,4$ \\
\hline & B & 501,0 & 509,6 & 531,6 & $-252,3$ & 506,8 & 514,6 & 536,6 & $-255,0$ \\
\hline & $\mathrm{C}$ & 576,7 & 582,4 & 604,4 & $-291,5$ & 561,3 & 567,8 & 589,8 & $-282,9$ \\
\hline & $\mathrm{D}$ & 506,5 & 513,9 & 535,9 & $-255,6$ & 503,8 & 512,7 & 534,7 & $-253,2$ \\
\hline \multirow[t]{4}{*}{ LWBN-UA } & $\mathrm{A}$ & $-552,9$ & $-543,1$ & $-521,1$ & 275,0 & $-575,0$ & $-557,6$ & $-536,7$ & 281,6 \\
\hline & B & $-538,6$ & $-528,2$ & $-506,2$ & 266,3 & $-557,9$ & $-548,9$ & $-526,9$ & 276,1 \\
\hline & $\mathrm{C}$ & $-434,7$ & $-425,6$ & $-403,6$ & 213,9 & $-490,2$ & $-483,5$ & $-461,6$ & 242,0 \\
\hline & $\mathrm{D}$ & $-434,9$ & $-425,1$ & $-403,1$ & 215,6 & $-466,1$ & $-456,3$ & $-434,3$ & 230,5 \\
\hline
\end{tabular}

Consideramos as amostras a posteriori dos parâmetros dos modelos LWBN-PA e LWBN-

UA e calculamos as estimativas de Monte Carlo das medidas de $\psi$-divergência descritas na Seção 1.4, com o objetivo de estudar as medidas consideradas na detecção das possíveis observações influentes na distribuição a posteriori dos modelos propostos.

As estimativas de Monte Carlo das medidas de $K$ - $L$ divergence, $L_{1}$-distance, $J$-distance e $\chi^{2}$-divergence são apresentadas na Tabela 3.5 e nas Figuras 3.3 e 3.4, mostrando que as observações influentes foram detectadas em cada modelo de ajuste. Verificamos claramente que todas as medidas tiveram um bom desempenho para identificar observações influentes e, de fato, 
as estimativas de Monte Carlo das medidas de $\psi$-divergência dos casos perturbados apresentaram os valores maiores em relação aos outros casos.

TABELA 3.5: Estimativas das medidas de $\psi$-divergência para os dados artificiais.

\begin{tabular}{ccccccc}
\hline Modelo & Configuração & $\begin{array}{c}\text { Identificação } \\
\text { dos casos }\end{array}$ & $d_{\mathrm{K}-\mathrm{L}}$ & $d_{\mathrm{J}}$ & $d_{L_{1}}$ & $d_{\chi^{2}}$ \\
\hline LWBN-PA & $\mathrm{A}$ & 45 & 0,0302 & 0,0606 & 0,0981 & 0,0631 \\
& $\mathrm{~B}$ & 350 & 0,0017 & 0,0034 & 0,0234 & 0,0034 \\
& $\mathrm{C}$ & 350 & 4,6921 & 5,1701 & 0,7181 & 436,4556 \\
& $\mathrm{D}$ & 45 & 0,7509 & 1,5741 & 0,4716 & 4,0828 \\
& & 350 & 1,5507 & 3,5841 & 0,6505 & 57,104 \\
\hline LWBN-UA & $\mathrm{A}$ & 45 & 0,0007 & 0,0017 & 0,0149 & 0,0014 \\
& & 350 & 0,0007 & 0,0015 & 0,0156 & 0,0015 \\
& $\mathrm{~B}$ & 45 & 3,3478 & 5,3579 & 0,7691 & 11,6334 \\
& $\mathrm{C}$ & 45 & 4,8699 & 8,2215 & 0,8722 & 70,3746 \\
& $\mathrm{D}$ & 350 & 3,5514 & 6,9089 & 0,8392 & 108,6190 \\
\hline
\end{tabular}



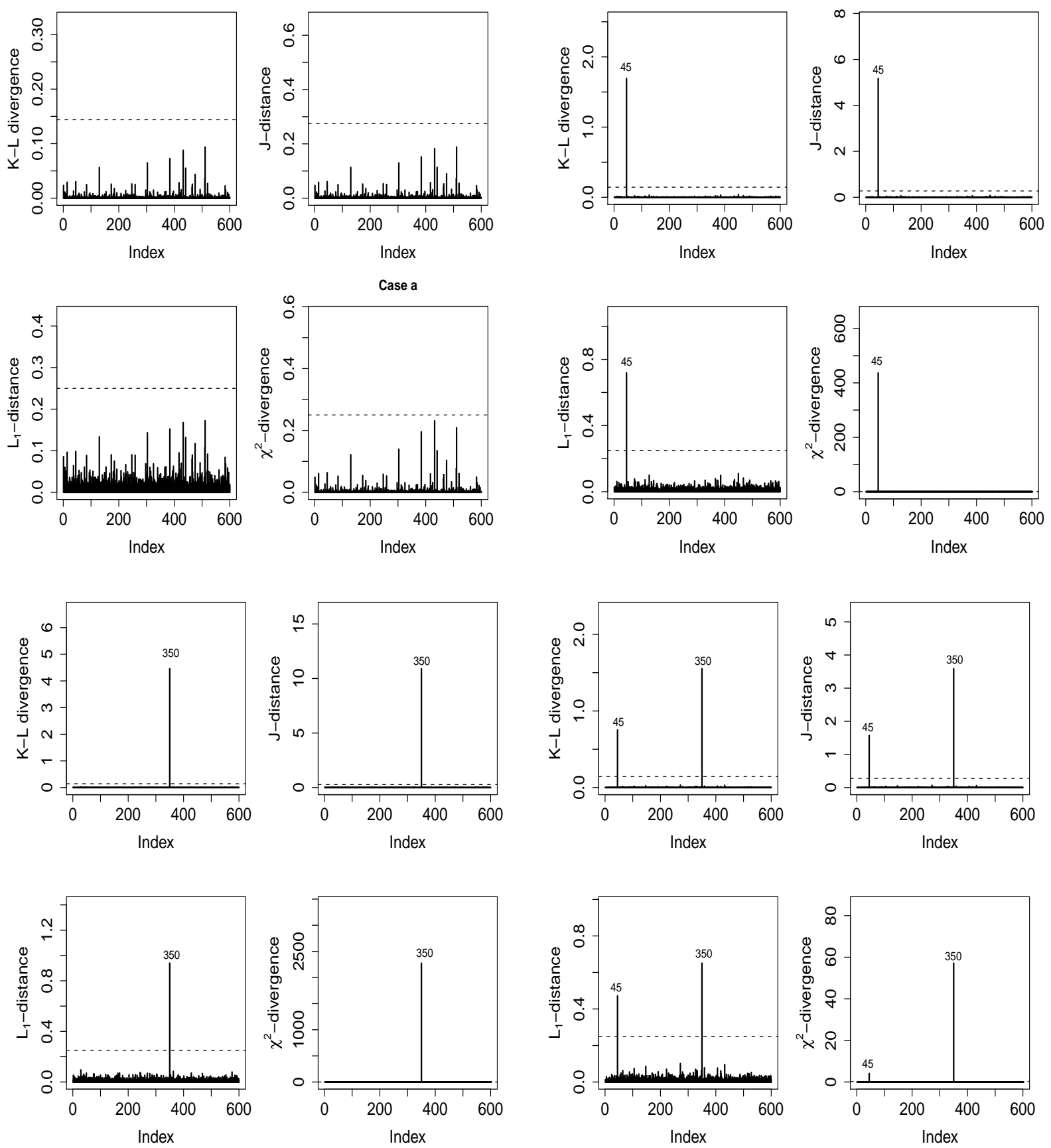

FIGURA 3.3: Estimativas de Monte Carlo das medidas de $\psi$-divergência do modelo de regressão LWBN-PA ajustado. Painel superior esquerdo: Configuração A, conjunto de dados sem perturbação. Painel superior direito: Configuração B, conjunto de dados com observação 45 perturbada. Painel inferior esquerdo: Configuração C, conjunto de dados com observação 350 perturbada. Painel inferior direito: Configuração D, conjunto de dados com observações 45 e 350 perturbadas. 

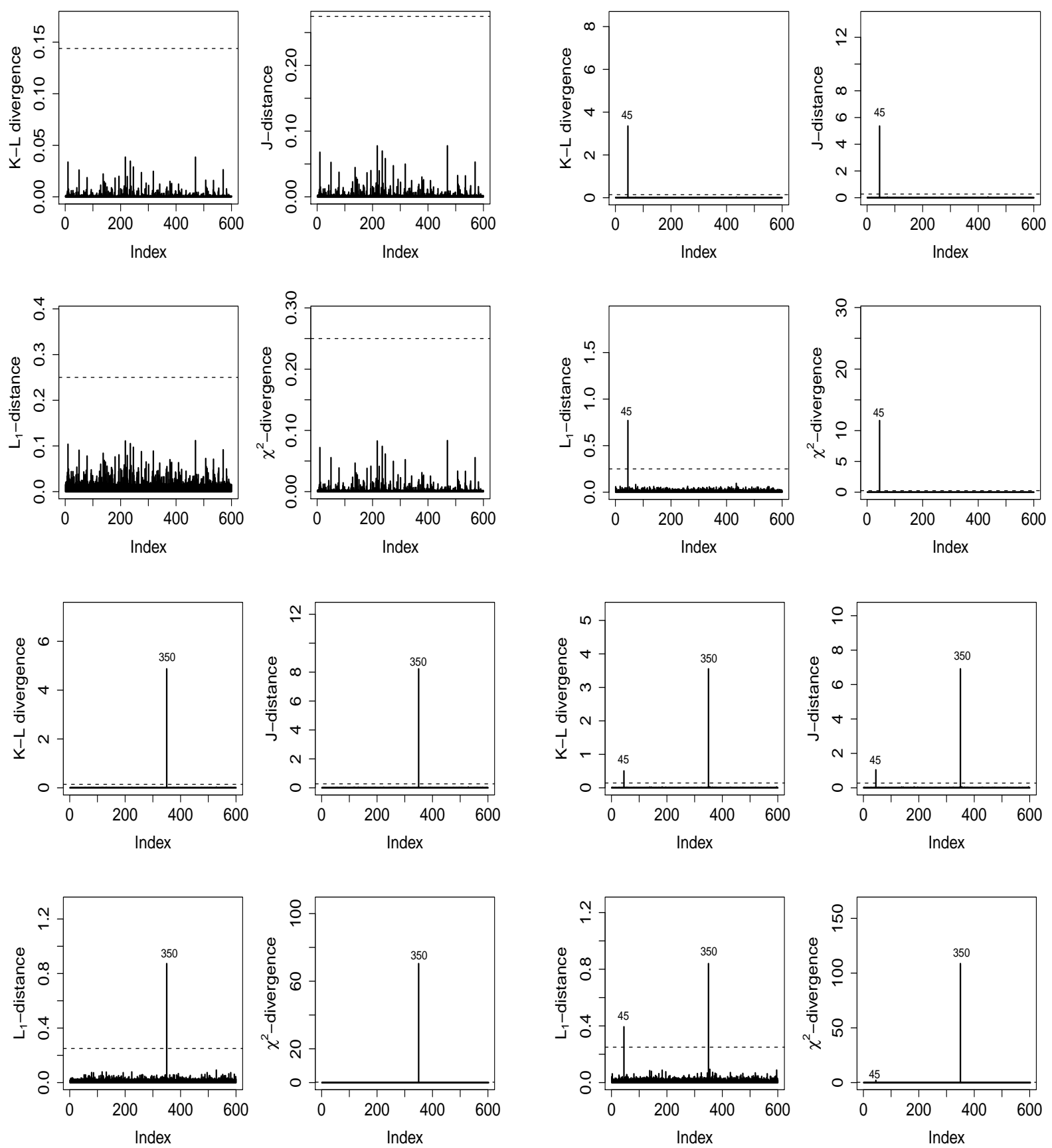

FIGURA 3.4: Estimativas de Monte Carlo das medidas de $\psi$-divergência do modelo de regressão LWBN-UA ajustado. Painel superior esquerdo: Configuração A, conjunto de dados sem perturbação. Painel superior direito: Configuração B, conjunto de dados com observação 45 perturbada. Painel inferior esquerdo: Configuração C, conjunto de dados com observação 350 perturbada. Painel inferior direito: Configuração D, conjunto de dados com observações 45 e 350 perturbadas. 


\subsection{Aplicação}

Nesta seção, um conjunto de dados reais foi utilizado para ajustar os modelos apresentados na Seção 3.2. Os dados referem-se aos números de adultos de Tribolium castaneum com 252 observações e Tribolium confusum com 690 observações cultivadas em $29^{\circ} \mathrm{C}$, apresentados por Eugene et al. (2002). Os modelos de regressão LWBN sob os esquemas de primeira ativação (LWBN-PA), última ativação (LWBN-UA) e ativação aleatória (LWBN-AA) foram ajustados para os dados.

De acordo com os valores dos critérios DIC, EAIC, EBIC, e LPML apresentados na Tabela 3.6, o modelo de regressão LWBN-PA sob o esquema de primeira ativação se apresenta como o melhor modelo ajustado, sendo selecionado como o modelo de trabalho. O resumo a posteriori dos parâmetros do modelo de regressão LWBN-PA é apresentado na Tabela 3.7, em que as médias a posteriori dos parâmetros do modelo, os respectivos erros padrão (EP) e os intervalos highest posterior density (HPD) (Chen \& Shao, 1998) com 95\% de credibilidade foram obtidos.

TABELA 3.6: Conjunto de dados reais. Critérios Bayesianos para modelos selecionados.

\begin{tabular}{lccc}
\hline & \multicolumn{3}{c}{ Ativação } \\
\cline { 2 - 4 } Critérios & Primeiro & Último & Aleatório \\
\hline DIC & 628,6610 & 675,9823 & 677,9761 \\
EAIC & 636,6785 & 687,6043 & 681,1316 \\
EBIC & 660,9026 & 711,8284 & 695,6661 \\
LPML & $-316,7407$ & $-340,3857$ & $-339,0835$ \\
\hline
\end{tabular}

TABELA 3.7: Conjunto de dados reais. Resumo a posteriori dos parâmetros do modelo de regressão LWBN-PA.

\begin{tabular}{ccccccc}
\hline \multirow{2}{*}{ Dados } & Parâmetro & Media & Mediana & EP & LI & LS \\
\hline Original & $\eta$ & 2,873 & 2,513 & 1,6147 & 0,522 & 5,911 \\
& $\theta$ & 12,251 & 11,036 & 5,7329 & 2,843 & 23,129 \\
& $\sigma$ & 0,194 & 0,193 & 0,0141 & 0,166 & 0,220 \\
& $\beta_{0}$ & 5,389 & 5,387 & 0,0416 & 5,304 & 5,473 \\
& $\beta_{1}$ & $-0,544$ & $-0,543$ & 0,0270 & $-0,596$ & $-0,492$ \\
\hline
\end{tabular}

Na esquerda da Figura 3.5 mostramos a função de distribuição acumulada ajustada pelo modelo de regressão LWBN-PA, que fornece uma boa aproximação da função de distribuição acumulada empírica (FDAE). Para detectar as possíveis observações influentes na distribuição 
a posteriori dos parâmetros do modelo, as estimativas das medidas de $\psi$-divergência foram obtidas pela amostra a posteriori dos parâmetros do modelo. O painel direito da Figura 3.5 mostra as estimativas de Monte Carlo das medidas de $K$ - $L$ divergence, $L_{1}$-distance, J-distance e $\chi^{2}$-divergence, onde as observações 686 e 687 são indicadas como possíveis observações influentes.
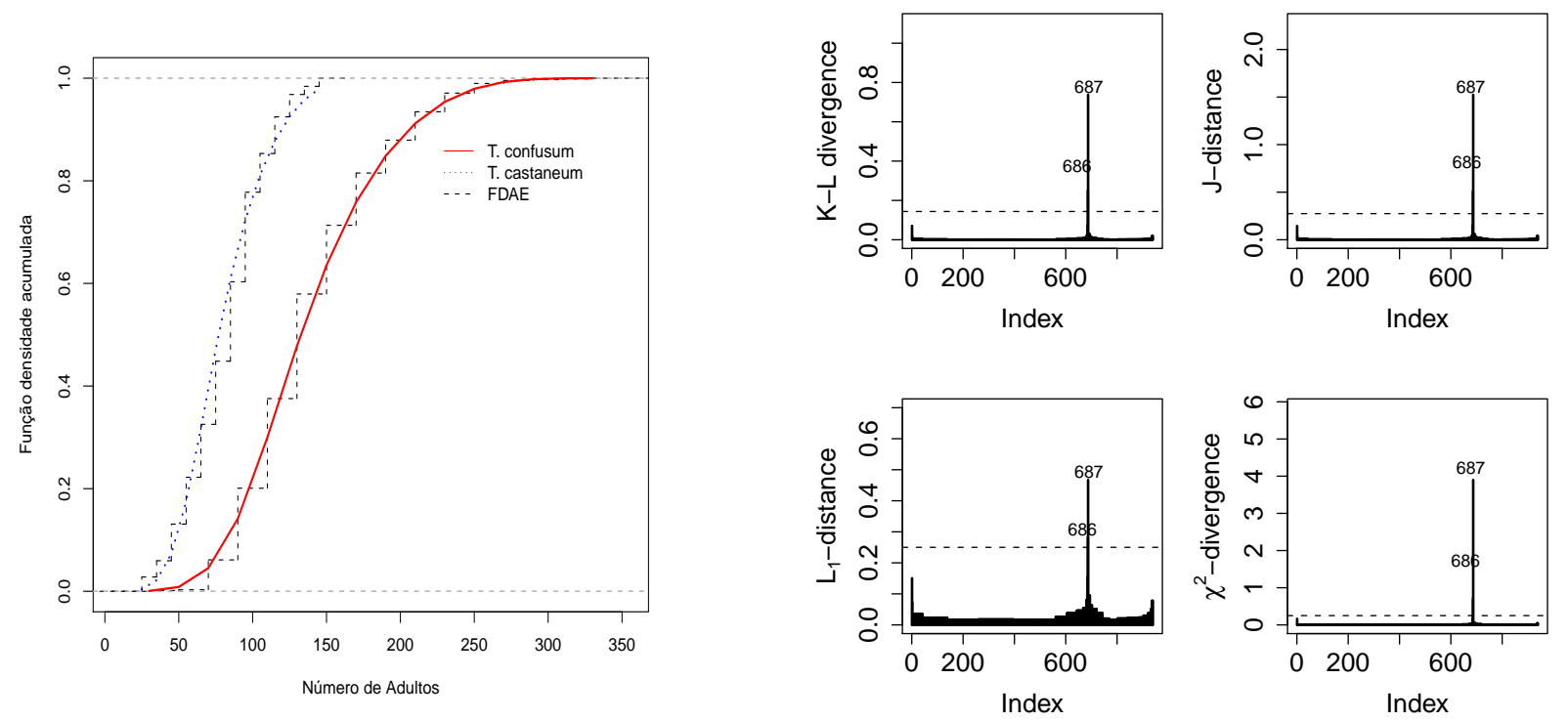

FIGURA 3.5: Dados originais. Painel esquerdo: Função densidade acumulada ajustada pelo modelo de regressão LWBN-PA. Painel direito: Estimativas de Monte Carlo das medidas de $\psi$-divergência do modelo de regressão LWBN-PA ajustado.

Para analisar o impacto dessas observações na distribuição a posteriori dos parâmetros do modelo LWBN-PA, reajustamos o modelo eliminando as observações 686 e 687; o resumo $a$ posteriori dos parâmetros do modelo é apresentado na Tabela 3.8. Note que apenas a média $a$ posteriori do parâmetro $\eta$ aumenta significativamente nesse caso.

O painel esquerdo da Figura 3.6 mostra que a fda ajustada pelo modelo de regressão LWBN-PA também fornece tem uma boa aproximação da FDAE. O painel direito da Figura 3.5 mostra as estimativas de Monte Carlo das quatro medidas consideradas, note que a observação 685 é indicada como a possível observação influente pelas medidas de $J$-distance e $\chi^{2}$-divergence, o que poderia ser mais investigado.

TABELA 3.8: Conjunto de dados reais. Resumo a posteriori dos parâmetros do modelo de regressão LWBN-PA.

\begin{tabular}{ccccccc}
\hline Sem & $\eta$ & 6,981 & 5,776 & 4,5215 & 0,959 & 16,326 \\
Observações & $\theta$ & 11,902 & 10,351 & 7,0346 & 1,580 & 25,658 \\
686 e 687 & $\sigma$ & 0,178 & 0,177 & 0,0148 & 0,150 & 0,207 \\
& $\beta_{0}$ & 5,356 & 5,357 & 0,0319 & 5,288 & 5,414 \\
& $\beta_{1}$ & $-0,549$ & $-0,549$ & 0,0256 & $-0,599$ & $-0,499$ \\
\hline
\end{tabular}



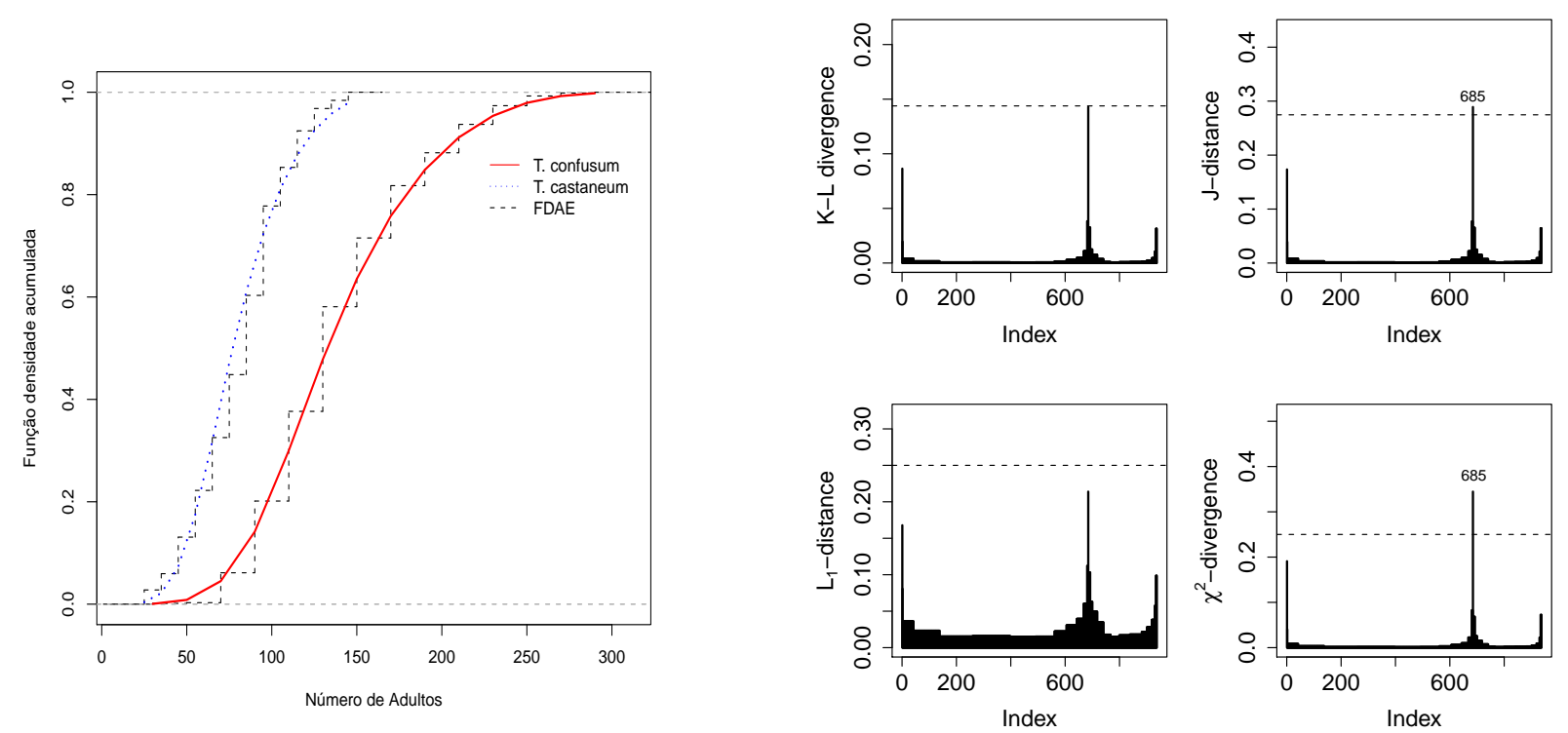

FIGURA 3.6: Dados reais sem observações 686 e 687. Painel esquerdo: Função densidade acumulada ajustada pelo modelo de regressão LWBN-PA. Painel direito: Estimativas de Monte Carlo das medidas de $\psi$-divergência do modelo de regressão LWBN-PA ajustado.

\subsection{Conclusão}

Neste trabalho, propomos o modelo de regressão LWBN sobre uma estrutura de ativação latente para explicar a ocorrência do evento de interesse; a inferência Bayesiana para os modelos propostos é baseada em métodos de MCMC. A análise de influência caso a caso é baseada nas medidas de $K$ - $L$ divergence, $L_{1}$-distance, $J$-distance e $\chi^{2}$-divergence, que possuem bom desempenho para identificar as observações influentes da simulação. Finalmente, um conjunto de dados real foi ajustado pelos modelos propostos para mostrar a potencialidade da metodologia. 


\section{Capítulo 4}

\section{Modelo de sobrevivência com fração}

\section{de cura}

\subsection{Introdução}

Na análise de dados de sobrevivência, determinados estudos se caracterizam por apresentar, em uma fração significativa de indivíduos, os que não mostram o evento de interesse, mesmo após um longo período de acompanhamento. Isso ocorre, por exemplo, em muitos estudos clínicos, especialmente em estudos de pessoas com câncer, nos quais há um número considerável de pacientes que respondem favoravelmente ao tratamento, apresentando uma proporção de indivíduos sobreviventes ou curados mesmo após um longo período de acompanhamento. Para tais dados de sobrevivência, verificamos que uma proporção de indivíduos da população é suscetível ao evento de interesse e outras não. Empiricamente, esta característica é observada nas estimativas de Kaplan \& Meier (1958) da função de sobrevivência, que apresenta uma cauda direita em um nível aproximadamente constante e estritamente maior que zero por um período considerável. Nesta situação, os modelos de sobrevivência usuais, os quais assumem que a função de sobrevivência converge para zero quando o tempo de sobrevivência é suficientemente grande (função de sobrevivência própria), podem não ser adequados.

Dados de sobrevivência que apresentam uma proporção de indivíduos da população não são suscetíveis ao evento de interesse; em geral, são modelados por meio dos modelos de sobrevivência com fração de cura. A literatura sobre modelos de fração de cura é extensa e está em rápido desenvolvimento. Destacando-se como referências fundamentais os trabalhos de Maller 
\& Zhou (1996) e Ibrahim et al. (2001), como também os artigos de Tsodikov et al. (2003), Cooner et al. (2007), Maller \& Zhou (1996), Ibrahim et al. (2001), Tsodikov et al. (2003), Cooner et al. (2007), Tournoud \& Ecochard (2007), Lopes et al. (2012), Rodrigues et al. (2009), Cancho et al. (2009), Cancho et al. (2011), Rodrigues et al. (2010a) e Rodrigues et al. (2010b).

A abordagem bastante usual para modelar dados de sobrevivência com fração de cura é o modelo de mistura proposto por Boag (1949), posteriormente desenvolvido por Berkson \& Gage (1952) e o modelo de tempo de promoção estudado por Yakovlev \& Tsodikov (1996) e Chen et al. (1999). No modelo de mistura, o número de causas do evento de interesse é uma variável aleatória binária em $\{0,1\}$, enquanto que, no modelo de tempo de promoção, este número segue uma Distribuição de Poisson. Neste capítulo propomos o modelo Weibull Binomial Negativo com fração de cura (WBNcr) em uma estrutura de riscos competitivos sobre diferentes mecanismos de ativação. Considerando os tempos não-observados, $T$ tem a distribuição Weibull com parâmetro de $\alpha$ e $\lambda$ dada em (1.1), e o número de causas é modelado pela distribuição Binomial Negativa, o qual inclui como caso particular o modelo de mistura e de tempo de promoção. Neste caso, assumindo que não há informação sobre qual fator foi responsável pelo acontecimento do evento de interesse, o tempo de vida somente é observado quando o R-ésimo fator de risco dentre $M$ foi observado.

Este capítulo está organizado da seguinte forma: na Seção 4.2, formulamos o modelo de sobrevivência com fração de cura, baseada na distribuição Binomial Negativa. Os procedimentos de inferência em uma perspectiva clássica e Bayesiana são apresentados nas Seções 4.3 e 4.5. Na Seção 4.4 é apresentado um estudo de simulação realizado com objetivo de comparar os modelos propostos WBNcr na presença dos diferentes esquemas de ativação. As aplicações com dados artificiais e reais são apresentadas nas Seções 4.6 e 4.7. Finalmente, a conclusão deste capítulo é apresentada na Seção 4.8 .

\subsection{Formulação do modelo}

Suponha que, para um indivíduo na população, seja $M$ uma variável aleatória discreta que representa o número de causas ou riscos de ocorrência de um particular evento de interesse. Assumindo que $M$ tem distribuição Binomial Negativa, com a função de probabilidade dada em (2.1) e função geradora de probabilidade dada por

$$
A_{p}(s)=[1+\eta \theta(1-s)]^{-1 / \eta}, \quad 0 \leq s \leq 1 .
$$


Dado $M=m, \operatorname{sejam} T_{j}, j=1,2, \ldots$ variáveis aleatórias contínuas não-negativas i.i.d. com função de distribuição acumulada $F(\cdot)=1-S(\cdot)$ e independentes de $M$, representando o tempo de ocorrência do evento de interesse devido à $j$-ésima causa ou risco, ou tempo de ativação devido à $j$-ésima causa. O tempo de ocorrência do evento de interesse observado pode ser definido pela variável aleatória $Y=T_{(R)}$, para $R=1, \cdots, M$, em que $T_{(1)} \leq \cdots \leq T_{(M)}$ são as estatísticas de ordem $T_{j}, R$ dependente de $M$ e $Y=\infty$ se $M=0$. Em muitos processos biológicos, $R$ indica fatores de resistência do sistema imunológico do indivíduo. Se o evento de interesse ocorrer (por exemplo, a reincidência do câncer), então a variável aleatória $Y$ tem o valor dado pela R-ésima estatística de ordem $T_{(R)}$. Em outras palavras, como em Cooner et al. (2006) e Cooner et al. (2007), $R$ de $M$ causas são necessários para produzir o evento de interesse. $R$ pode ser uma constante fixa, em função de $M$ ou uma variável aleatória especificada através de uma distribuição condicional $M$.

Usando a terminologia empregada em Cooner et al. (2006) e Cooner et al. (2007), neste trabalho consideramos três casos específicos para $R$. Primeiro, assumindo que, dado $M \geq 1$ a distribuição condicional de $R$ é uma variável aleatória discreta uniforme em $\{1,2, \cdots, M\}$ com probabilidade $1 / M$, então a função de sobrevivência de $Y$ é dada por

$$
\begin{aligned}
S_{\mathrm{pop}}(y) & =P(Y \geq y)=P(M=0)+P\left(T_{(R)}>y, 1 \leq R \leq M\right) \\
& =P(M=0)+\sum_{k=1}^{\infty} \sum_{R=1}^{k} P\left(T_{(R)}>y \mid R, M=k\right) P(R \mid M=k) P(M=k)
\end{aligned}
$$

em que

$$
P\left(T_{(R)}>y \mid R, M=k\right)=\sum_{i=0}^{R-1}\left(\begin{array}{l}
k \\
i
\end{array}\right) F(y)^{i} S(y)^{k-i},
$$

com $S(y)=1-F(y)$. Note que (4.3) é a função de distribuição acumulativa da distribuição binomial com k ensaios e probabilidade de sucesso $F(y)$. Substituindo (4.3) em (4.2), temos que a função de sobrevivência de $Y$ é dada por 


$$
\begin{aligned}
S_{\mathrm{pop}}(y) & =P(M=0)+\sum_{k=1}^{\infty}\left\{\sum_{R=0}^{k}(k-R) B(R ; k, F(y))\right\} \frac{1}{k} P(M=k) \\
& =P(M=0)+S(y) \sum_{k=1}^{\infty} P(M=k) \\
& =(1+\eta \theta)^{-1 / \eta}+\left(1-(1+\eta \theta)^{-1 / \eta}\right) S(y)
\end{aligned}
$$

em que $B(x ; k, F(y))=P(X=x)$ e $X \sim \operatorname{Binomial}(k, F(y))$. O modelo em (4.4) vem a ser um modelo de mistura padrão (Berkson \& Gage, 1952) com a fração de curados $p_{0}=P(M=0)=$ $\lim _{y \rightarrow \infty} S_{\mathrm{pop}}(y)=(1+\eta \theta)^{-1 / \eta}$. A função de densidade correspondente ao (4.4) é dada por

$$
f_{\mathrm{pop}}(y)=-S_{\mathrm{pop}}^{\prime}(y)=\left(1-(1+\eta \theta)^{-1 / \eta}\right) f(y),
$$

onde $f(y)=-S^{\prime}(y)$ denota a função densidade do tempo de ocorrência do evento de interesse $T$ em (4.4). Além disso, a função de risco correspondente é dada por

$$
h_{\mathrm{pop}}(y)=\frac{f(t)\left((1+\eta \theta)^{-1 / \eta}\right)}{(1+\eta \theta)^{-1 / \eta}+S(t)\left(1-(1+\eta \theta)^{-1 / \eta}\right)}
$$

Na segunda configuração, supomos que o evento de interesse ocorre porque a primeira das possíveis causas é ativada, o que é conhecido como mecanismo de primeira ativação. Neste caso, $R=1$ e o tempo de vida observado é definido pela variável aleatória $Y=\min \left\{T_{1}, \cdots, T_{M}\right\}$. Sob essa configuração, considerando (4.2), podemos demonstrar que a função de sobrevivência de $Y$ é dada por

$$
S_{\mathrm{pop}}=P(Y \geq y)=A_{p}(S(y))
$$

que é o modelo proposto por Rodrigues et al. (2008), em que $A_{p}(\cdot)$ é a função geradora de probabilidade da variável aleatória $M$. 
Prova:

$$
\begin{aligned}
S_{\mathrm{pop}}(y) & =P[\text { não ocorrer o evento de interesse até o tempo } y] \\
& =P\left[T_{0}>y, M=0\right]+P\left[T_{1}>y, T_{2}>y, \ldots, T_{M}>y, M \geq 1\right] \\
& =p_{0}+P\left[T_{1}>y, T_{2}>y, \ldots, T_{M}>y, M \geq 1\right] \\
& =p_{0}+\sum_{m=1}^{\infty} P[M=m] P\left[T_{1}>y, T_{2}>y, \ldots, T_{M}>y \mid M=m\right] \\
& =p_{0}+\sum_{m=1}^{\infty} P[M=m]\{S(y)\}^{m} \\
& =A_{p}(S(y)) .
\end{aligned}
$$

Considerando (4.1), temos que a função de sobrevivência populacional é dada por

$$
S_{\text {pop }}(y)=(1+\eta \theta F(y))^{-1 / \eta}
$$

de modo que a fração de cura é dada por $p_{0}=\lim _{y \rightarrow \infty} S_{\text {pop }}(y)=(1+\theta \eta)^{-1 / \eta}$. A função de densidade correspondente a (4.8) é dada por

$$
f_{\mathrm{pop}}(y)=\theta f(y)(1+\eta \theta F(t))^{(1+1 / \eta)}
$$

note que $f_{\text {pop }}(y)$ é uma função de densidade imprópria, uma vez que $S_{\text {pop }}(y)$ é uma função de sobrevivência imprópria. Além disso, a função de risco correspondente é dada por

$$
h_{\text {pop }}(y)=\theta f(y)(1+\theta \eta F(y))^{-1} .
$$

Na terceira configuração, o evento de interesse ocorre somente depois que todas as $M$ causas são ativadas, fato também conhecido como esquema da última ativação. Neste caso, $R=M$ e o tempo de vida observado é dado por $Y=\max \left\{T_{1}, \cdots, T_{M}\right\}$. Sob essa configuração, considerando (4.2), podemos demonstrar que a função de sobrevivência de $Y$ é dada por

$$
S_{p o p}(y)=1+A_{p}(0)-A_{p}(F(t))
$$


Prova:

$$
\begin{aligned}
S_{\text {pop }}(y) & =P[\text { não ocorrer o evento de interesse até o tempo } y] \\
& =P\left[T_{0}>y, M=0\right]+\sum_{m=1}^{\infty} P[M=m]\left(1-P\left[\max \left\{T_{1}, \ldots, T_{M}\right\} \leq y\right]\right) \\
& =P[M=0]+\sum_{m=1}^{\infty} P[M=m]-\sum_{m=1}^{\infty} P[M=m] P\left[T_{1} \leq y, \ldots, T_{M} \leq y \mid M=m\right] \\
& =A_{p}(0)+\sum_{m=0}^{\infty} P[M=m]-P[M=0]-\sum_{m=1}^{\infty} P[M=m] P\left[T_{1} \leq y, \ldots, T_{M} \leq y \mid M=m\right] \\
& =A_{p}(0)+1-P[M=0]-\sum_{m=1}^{\infty} P[M=m] F(y)^{m} \\
& =A_{p}(0)+1-\sum_{m=0}^{\infty} P[M=m] F(y)^{m} \\
& =1+A_{p}(0)-A_{p}(F(y)) .
\end{aligned}
$$

Assim, de (4.1) temos que a função de sobrevivência populacional é dada por

$$
S_{\mathrm{pop}}(y)=1+(1+\theta \eta)^{-1 / \eta}-(1+\theta \eta S(y))^{-1 / \eta}
$$

de modo que a fração de cura é dada por $p_{0}=\lim _{y \rightarrow \infty} S_{\text {pop }}(y)=(1+\theta \eta)^{-1 / \eta}$. A função de densidade correspondente a (4.12) é dada por

$$
f_{\mathrm{pop}}(y)=\theta f(y)(1+\theta \eta S(t))^{-(1 / \eta+1)}
$$

com função de risco

$$
h_{\mathrm{pop}}(y)=\frac{\theta f(y)(1+\theta \eta S(t))^{-(1 / \eta+1)}}{1+(1+\theta \eta)^{-1 / \eta}-(1+\theta \eta S(y))^{-1 / \eta}} .
$$

Por meio desses resultados observamos que, seja qual for o esquema de ativação, a fração de cura é a mesma, porém os modelos diferem nas suas funções de sobrevivência, de densidade e de risco.

A seguinte proposição mostra as relações entre as funções distribuições dadas por (4.4), (4.8) e (4.12) denotadas, respectivamente, por $S_{\text {pop }}^{a}(y), S_{\text {pop }}^{p}(y)$ e $S_{\text {pop }}^{u}(y)$.

Proposição 4.1. Sob condições dos modelos em (4.4), (4.8), e (4.12) para qualquer função de distribuição $G(y)$, temos que $S_{\text {pop }}^{p}(y) \leq S_{\text {pop }}^{a}(y) \leq S_{\text {pop }}^{u}(y)$ para todo $y>0$.

Prova. Sabemos que $\frac{1-(1+\eta \theta S(y))^{-1 / \eta}}{S(y)}$ é uma função crescente em relação a $y$, e que podemos obter o valor mínimo quando $y=0$, isto é, $\lim _{y \rightarrow 0} \frac{1-(1+\eta \theta S(y))^{-1 / \eta}}{S(y)}=1-(1+\theta \eta)^{-1 / \eta}$, $\Rightarrow \frac{1-(1+\eta \theta S(y))^{-1 / \eta}}{S(y)} \geq 1-(1+\theta \eta)^{-1 / \eta}, \forall y$. Logo, $1-(1+\eta \theta S(y))^{-1 / \eta} \geq S(y)\left(1-(1+\eta \theta)^{-1 / \eta}\right)$ 
$\Rightarrow 1+\left(1_{\theta} \eta\right)^{-1 / \eta}-(1+\eta \theta S(y))^{-1 / \eta} \geq 1+\left(1_{\theta} \eta\right)^{-1 / \eta}+S(y)\left(1-(1+\eta \theta)^{-1 / \eta}\right)$, o que implica que $S_{\text {pop }}^{u}(y) \geq S_{\text {pop }}^{a}(y)$. Analogamente para provar $S_{\text {pop }}^{p} \leq S_{\text {pop }}^{a}$.

Em muitas aplicações de modelos de sobrevivência de longa duração, a fração de cura desempenha um papel central, e por essa causa consideramos a parametrização do modelo em fração de cura $p_{0}$ nas expressões. Já que $p_{0}=P(M=0)=(1+\eta \theta)^{-1 / \eta}$, temos que $\theta=\left(p_{0}^{-\eta}-1\right) / \eta$. Usando $p_{0}$ como parâmetro, e assumindo os tempos não-observados $T$ com a distribuição Weibull com parâmetro de $\alpha$ e $\lambda$ dada em (1.1), de (4.4)-(4.14), obtemos as funções de sobrevivência imprópria e as funções de densidade para modelo WBN com fração de cura sobre três diferentes esquemas de ativação, que são apresentados na Tabela 4.1, na qual $f(y), F(y)$ e $S(y)$ são a $f d p, f d a$ e funções de sobrevivência da distribuição Weibull dada em (1.1).

TABELA 4.1: Função de Sobrevivência $S_{\text {pop }}(y)$ e $f d p f_{\text {pop }}(y)$ para o modelo WBN com fração de cura sobre três diferentes esquemas de ativação

\begin{tabular}{lcc}
\hline Modelo (Ativação) & $S_{\text {pop }}(y)$ & $f_{\text {pop }}(y)$ \\
\hline $\begin{array}{l}\text { WBNcr-PA } \\
\text { (Primeira) }\end{array}$ & {$\left[1+\left(p_{0}^{-\eta}-1\right) F(y)\right]^{-1 / \eta}$} & $\left(p_{0}^{-\eta}-1\right) f(y)\left[1+\left(p_{0}^{-\eta}-1\right) F(y)\right]^{-(1+1 / \eta)} / \eta$ \\
$\begin{array}{l}\text { WBNcr-UA } \\
\text { (Última) }\end{array}$ & $1+p_{0}-\left[1+\left(p_{0}^{-\eta}-1\right) S(y)\right]^{-1 / \eta}$ & $\left(p_{0}^{-\eta}-1\right) f(y)\left[1+\left(p_{0}^{-\eta}-1\right) S(y)\right]^{-(1+1 / \eta)} / \eta$ \\
$\begin{array}{l}\text { WBNcr-AA } \\
\text { (Aleatória) }\end{array}$ & $p_{0}+S(y)\left(1-p_{0}\right)$ & $f(y)\left(1-p_{0}\right)$ \\
\hline
\end{tabular}

A Figura 4.1 mostrou os comportamentos distintos das funções de sobrevivência da Tabela 4.1, ilustrando a flexibilidade do modelo proposto.

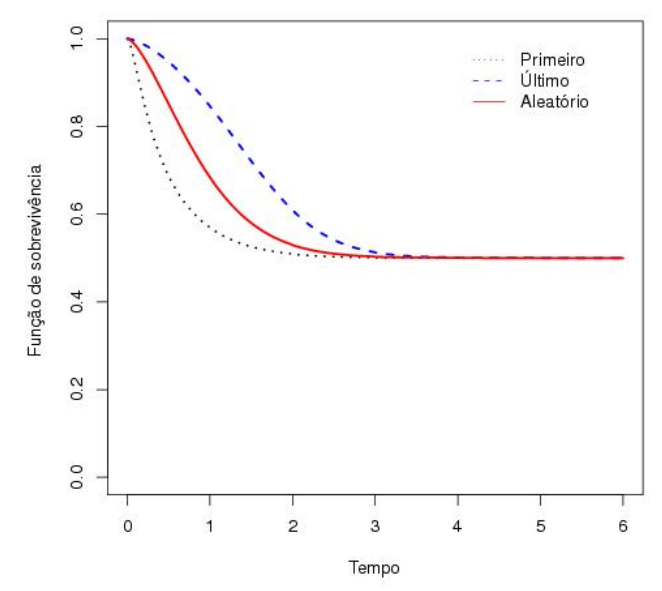

FIGURA 4.1: Função de sobrevivência da distribuição WBN com fração de cura sobre três diferentes esquemas de ativação com parâmetros $\lambda=0, \alpha=1.5, \eta=3$ e $p_{0}=0.5$. 


\subsection{Inferência Clássica}

Considere a situação em que os tempos de ocorrer o evento de interesse $y_{i}$ dados na Tabela 4.1 não são totalmente observados e estão sujeitos à censura à direita. Então, seja $z_{i}$ é o tempo observado tal que $z_{i}=\min \left\{y_{i}, c_{i}\right\}$, com $c_{i}$ sendo o tempo de censura e $\delta_{i}=I\left(y_{i} \leq c_{i}\right)$ sendo um indicador de censura, i.e., quando $\delta_{i}=1$ se $z_{i}$ for o tempo de falha e $\delta_{i}=0$ se ele é censurado à direita, para $i=1, \cdots, n$.

Seja $\mathbf{x}_{i}=\left(x_{i 1}, \cdots, x_{i p}\right)^{\top}$ o vetor de covariáveis para o $i$-ésimo indivíduo. Completando o nosso modelo, propomos para relacionar a fração de cura com as covariáveis, utilizando a ligação logística

$$
\log \left(\frac{p_{0 i}}{1-p_{0 i}}\right)=\mathbf{x}_{i}^{\top} \boldsymbol{\beta} \quad \text { ou } \quad p_{0 i}=\frac{\exp \left(\mathbf{x}_{i}^{\top} \boldsymbol{\beta}\right)}{1+\exp \left(\mathbf{x}_{i}^{\top} \boldsymbol{\beta}\right)}
$$

em que $\boldsymbol{\beta}=\left(\beta_{0}, \cdots, \beta_{p}\right)^{\top}$ denota o vetor dos coeficientes de regressão. Consideramos os dados com $n$ pares de tempo de evento e indicador de censura dado por $\left\{\left(z_{1}, \delta_{1}\right), \cdots,\left(z_{n}, \delta_{n}\right)\right\}$. A função de verossimilhança com censura não informativa é dada por

$$
L(\boldsymbol{\vartheta} ; \mathcal{D}) \propto \prod_{i=1}^{n} f_{\mathrm{pop}}\left(z_{i} ; \boldsymbol{\vartheta}\right)^{\delta_{i}} S_{\mathrm{pop}}\left(z_{i} ; \boldsymbol{\vartheta}\right)^{1-\delta_{i}}
$$

em que $\boldsymbol{\vartheta}=\left(\alpha, \lambda, \eta, \boldsymbol{\beta}^{\top}\right)^{\top}$ representa o vetor dos parâmetros e $\mathcal{D}=\left(n, \mathbf{Z}, \boldsymbol{\delta}, \mathbf{x}_{i}\right)$ são os dados observados, com $\mathbf{z}=\left(z_{1}, \ldots, z_{n}\right)^{\top}, \boldsymbol{\delta}=\left(\delta_{1}, \ldots, \delta_{n}\right)^{\top}$ e $\mathbf{x}_{i}=\left(x_{i 1}, \cdots, x_{i n}\right)^{\top}$, considerando que $f_{\mathrm{pop}}(\cdot ; \boldsymbol{\vartheta})$ e $S_{\mathrm{pop}}(\cdot ; \boldsymbol{\vartheta})$ são as funções de densidade e sobrevivência imprópria na Tabela 4.1.

Seja $y_{1}, \cdots, y_{n}$ uma amostra aleatória de tamanho $n$ da distribuição WBNcr-PA com vetor de parâmetros $\boldsymbol{\vartheta}=\left(\alpha, \lambda, \eta, \boldsymbol{\beta}^{\top}\right)$. A partir de (4.16) a função de log-verossimilhança para $\boldsymbol{\vartheta}$ é dada por

$$
\ell=r \log \left(p_{0 i}^{-\eta}-1\right)-r \log \eta+\sum_{i=1}^{n} \delta_{i} \log \left[f_{\mathrm{W}}\left(z_{i}\right)\right]-\sum_{i=1}^{n}\left(\delta_{i}+1 / \eta\right) \log T_{1,0,1}^{(i)}
$$

em que $r=\sum_{i=1}^{n} \delta_{i}, f_{\mathrm{W}}\left(z_{i}\right), F_{\mathrm{W}}\left(z_{i}\right)$ e $S_{\mathrm{W}}\left(z_{i}\right)$ denotam as $f d p, f d a$ e a função de sobrevivência da distribuição Weibull dada na Seção 1.2, e

$$
T_{j, k, l}^{(i)}=\left[1+\left(p_{0}^{-\eta}-1\right) F_{W}\left(z_{i}\right)^{j} S_{W}\left(z_{i}\right)^{k}\right]^{l}, \quad j, k, l \in \mathbb{R}, \quad i=1 \cdots n .
$$


Os componentes do vetor escore $U(\boldsymbol{\vartheta})=\left(\partial \ell / \partial \alpha, \partial \ell / \partial \lambda, \partial \ell / \partial \eta, \partial \ell / \partial \boldsymbol{\beta}^{\top}\right)^{\top}$ são dados por

$$
\begin{aligned}
\frac{\partial \ell}{\partial \alpha} & =\sum_{i=1}^{n} \frac{\delta_{i}}{f_{\mathrm{W}}\left(z_{i}\right)} \frac{\partial f_{\mathrm{W}}\left(z_{i}\right)}{\partial \alpha}-\sum_{i=1}^{n}\left(\delta_{i}+1 / \eta\right) T_{1,0,-1}^{(i)}\left(p_{0 i}^{-\eta}-1\right) \frac{\partial F_{\mathrm{W}}\left(z_{i}\right)}{\partial \alpha} ; \\
\frac{\partial \ell}{\partial \lambda} & =\sum_{i=1}^{n} \frac{\delta_{i}}{f_{\mathrm{W}}\left(z_{i}\right)} \frac{\partial f_{\mathrm{W}}\left(z_{i}\right)}{\partial \lambda}-\sum_{i=1}^{n}\left(\delta_{i}+1 / \eta\right) T_{1,0,-1}^{(i)}\left(p_{0 i}^{-\eta}-1\right) \frac{\partial F_{\mathrm{W}}\left(z_{i}\right)}{\partial \lambda} \\
\frac{\partial \ell}{\partial \eta} & =-\frac{r p_{0 i}^{-\eta} \log p_{0 i}}{p_{0 i}^{-\eta}-1}-\frac{r}{\eta}-\sum_{i=1}^{n} \eta^{-2} \log \left[T_{1,0,1}^{(i)}\right]-F_{\mathrm{W}}\left(z_{i}\right) \eta^{-1} p_{0 i}^{-\eta} \log \left(p_{0 i} T_{1,0,-1}^{(i)}\right) \\
\frac{\partial \ell}{\partial \beta_{j}} & =-\frac{r \eta p_{0 i}^{-(r+1)}}{p_{0 i}^{-\eta}-1} \frac{\partial p_{0 i}}{\partial \beta_{j}}+\sum_{i=1}^{n}\left(\eta \delta_{i}+1\right) F_{\mathrm{W}}\left(z_{i}\right) p_{0 i}^{-(\eta+1)} T_{1,0,-1}^{(i)} \frac{\partial p_{0 i}}{\partial \beta_{j}} ; j=0, \cdots, p,
\end{aligned}
$$

em que

$$
\begin{aligned}
\frac{\partial f_{\mathrm{W}}\left(z_{i}\right)}{\partial \alpha} & =z_{i}^{\alpha-1} \exp \left(\lambda-z_{i}^{\alpha} e^{\lambda}\right)\left(1+\alpha(\alpha-1) z_{i}^{-1}-\alpha^{2} z_{i}^{\alpha-1} e^{\lambda}\right) ; \\
\frac{\partial f_{\mathrm{W}}\left(z_{i}\right)}{\partial \lambda} & =\exp \left(\lambda-z_{i}^{\alpha} e^{\lambda}\right)\left(\alpha z_{i}^{\alpha-1}-t_{i}^{\alpha} e^{\lambda}\right) ; \\
\frac{\partial F_{\mathrm{W}}\left(z_{i}\right)}{\partial \alpha} & =z_{i}^{\alpha} \log \left(z_{i}\right) \exp \left(2 \lambda-z_{i}^{\alpha} e^{\lambda}\right) ; \\
\frac{\partial F_{\mathrm{W}}\left(z_{i}\right)}{\partial \lambda} & =\exp \left(\lambda-z_{i}^{\alpha} e^{\lambda}\right)\left(y^{\alpha} e^{\lambda}-1\right) . \\
\frac{\partial p_{0 i}}{\partial \beta_{j}} & =\frac{\exp \left(\boldsymbol{x}_{\boldsymbol{i}}^{\top} \boldsymbol{\beta}\right) x_{i j}}{\left(1+\exp \left(\boldsymbol{x}_{i}^{\top} \boldsymbol{\beta}\right)\right)^{2}} ; j=1, \cdots, p .
\end{aligned}
$$

Agora, seja $y_{1}, \cdots, y_{n}$ uma amostra aleatória de tamanho $n$ da distribuição WBNcr-UA com vetor de parâmetros $\boldsymbol{\vartheta}=\left(\alpha, \lambda, \eta, \boldsymbol{\beta}^{\top}\right)$. De acordo com (4.21), a função de log-verossimilhança para $\vartheta$ é dada por

$$
\begin{aligned}
\ell= & r \log \left(p_{0 i}^{-\eta}-1\right)-r \log \eta+\sum_{i=1}^{n} \delta_{i} \log \left[f_{\mathrm{W}}\left(z_{i}\right)\right]-\left(1+\eta^{-1}\right) \sum_{i=1}^{n} \delta_{i} \log T_{0,1,1}^{(i)} \\
& +\sum_{i=1}^{n}\left(1-\delta_{i}\right) \log \left(1-p_{0 i}-T_{0,1,-1 / \eta}^{(i)}\right)
\end{aligned}
$$


Os componentes do vetor escore $U(\boldsymbol{\vartheta})=\left(\partial \ell / \partial \alpha, \partial \ell / \partial \lambda, \partial \ell / \partial \eta, \partial \ell / \partial \boldsymbol{\beta}^{\top}\right)^{\top}$ são dados por

$$
\begin{aligned}
\frac{\partial \ell}{\partial \alpha}= & \sum_{i=1}^{n} \frac{\delta_{i}}{f_{\mathrm{W}}\left(z_{i}\right)} \frac{\partial f_{\mathrm{W}}\left(z_{i}\right)}{\partial \alpha}-(1+1 / \eta) \sum_{i=1}^{n} \delta_{i} T_{0,1,-1}^{(i)}\left(p_{0 i}^{-\eta}-1\right) \frac{\partial S_{\mathrm{W}}\left(z_{i}\right)}{\partial \alpha}+\sum_{i=1}^{n}\left(1-\delta_{i}\right)\left(p_{0 i}-1\right) \eta^{-1} \\
& {\left[1-p_{0 i}-T_{0,1,-1 / \eta}^{(i)}\right]^{-1} T_{0,1,-1 / \eta-1}^{(i)} \frac{\partial S_{\mathrm{W}}\left(z_{i}\right)}{\partial \alpha} ; } \\
\frac{\partial \ell}{\partial \lambda}= & \sum_{i=1}^{n} \frac{\delta_{i}}{f_{\mathrm{W}}\left(z_{i}\right)} \frac{\partial f_{\mathrm{W}}\left(z_{i}\right)}{\partial \lambda}-(1+1 / \eta) \sum_{i=1}^{n} \delta_{i} T_{0,1,-1}^{(i)}\left(p_{0 i}^{-\eta}-1\right) \frac{\partial S_{\mathrm{W}}\left(z_{i}\right)}{\partial \lambda}+\sum_{i=1}^{n}\left(1-\delta_{i}\right)\left(p_{0 i}-1\right) \eta^{-1} \\
& {\left[1-p_{0 i}-T_{0,1,-1 / \eta}^{(i)}\right]^{-1} T_{0,1,-1 / \eta-1}^{(i)} \frac{\partial S_{\mathrm{W}}\left(z_{i}\right)}{\partial \lambda} ; } \\
\frac{\partial \ell}{\partial \eta}= & -\frac{r p_{0 i}^{-\eta} \log p_{0 i}}{p_{0 i}^{-\eta}-1}-\frac{r}{\eta}+\eta^{-2} \sum_{i=1}^{n} \log T_{0,1,1}^{(i)}+\eta^{-1} \sum_{i=1}^{n} \delta_{i} S_{\mathrm{W}}\left(t_{i}\right) p_{0}^{-\eta} \log p_{0 i} T_{0,1,-1} \\
& -\sum_{i=1}^{n} \frac{\left(1-\delta_{i}\right) p_{0 i}^{-\eta} \log p_{0 i} S_{W}\left(z_{i}\right) T_{0,1,-(1+1 / \eta)}^{(i)} ;}{\eta\left(1-p_{0 i}-T_{0,1,-1 / \eta}^{(i)}\right)} \\
\frac{\partial \ell}{\partial \beta_{j}}= & -\frac{r \eta p_{0 i}^{-(r+1)}}{p_{0 i}^{-\eta}-1} \frac{\partial p_{0 i}}{\partial \beta_{j}}+\sum_{i=1}^{n}(\eta+1) \delta_{i} S_{\mathrm{W}}\left(z_{i}\right) p_{0 i}^{-(\eta+1)} T_{0,1,-1}^{(i)} \frac{\partial p_{0 i}}{\partial \beta_{j}} \\
& +\sum_{i=1}^{n}\left(1-\delta_{i}\right) p_{0 i}^{-(\eta+1)} S_{\mathrm{W}}\left(z_{i}\right)\left(1-T_{0,1,-(1+1 / \eta)}^{(i)}\right) \frac{\partial p_{0 i}}{\partial \beta_{j}}\left[1-p_{0 i}+T_{0,1,-1 / \eta}^{(i)}\right]^{-1}, j=0, \cdots, p .
\end{aligned}
$$

em que $\partial S_{\mathrm{W}}\left(z_{i}\right) / \partial \alpha=-\partial F_{\mathrm{W}}\left(z_{i}\right) / \partial \alpha$ e $\partial S_{\mathrm{W}}\left(z_{i}\right) / \partial \lambda=-\partial F_{\mathrm{W}}\left(z_{i}\right) / \partial \lambda$.

Seja $y_{1}, \cdots, y_{n}$ uma amostra aleatória de tamanho $n$ da distribuição WBNcr-AA com vetor de parâmetros $\boldsymbol{\vartheta}=\left(\alpha, \lambda, \boldsymbol{\beta}^{\top}\right)$. De acordo com (4.21), a função de log-verossimilhança para $\vartheta$ é dada por

$$
\ell=\sum_{i=1}^{n} \delta_{i} \log f_{\mathrm{W}}\left(z_{i}\right)+\sum_{i=1}^{n} \delta_{i} \log \left(1-p_{0 i}\right)+\sum_{i=1}^{n}\left(1-\delta_{i}\right) \log \left(p_{0 i}+S_{\mathrm{W}}\left(z_{i}\right)\left(1-p_{0 i}\right)\right) .
$$

Os componentes do vetor escore $U(\boldsymbol{\vartheta})=\left(\partial \ell / \partial \alpha, \partial \ell / \partial \lambda, \partial \ell / \partial \boldsymbol{\beta}^{\top}\right)^{\top}$ são dados por

$$
\begin{aligned}
\frac{\partial \ell}{\partial \alpha} & =\sum_{i=1}^{n} \frac{\delta_{i}}{f_{\mathrm{W}}\left(z_{i}\right)} \frac{\partial f_{\mathrm{W}}\left(z_{i}\right)}{\partial \alpha}+\sum_{i=1}^{n} \frac{\left(1-\delta_{i}\right)\left(1-p_{0 i}\right)}{p_{0 i}+S_{W}\left(z_{i}\right)\left(1-p_{0 i}\right)} \frac{\partial S_{\mathrm{W}}\left(z_{i}\right)}{\partial \alpha} ; \\
\frac{\partial \ell}{\partial \lambda} & =\sum_{i=1}^{n} \frac{\delta_{i}}{f_{\mathrm{W}}\left(z_{i}\right)} \frac{\partial f_{\mathrm{W}}\left(z_{i}\right)}{\partial \lambda}+\sum_{i=1}^{n} \frac{\left(1-\delta_{i}\right)\left(1-p_{0 i}\right)}{p_{0 i}+S_{\mathrm{W}}\left(z_{i}\right)\left(1-p_{0 i}\right)} \frac{\partial S_{\mathrm{W}}\left(z_{i}\right)}{\partial \lambda} \\
\frac{\partial \ell}{\partial \beta_{j}} & =-\frac{\partial p_{0 i}}{\partial \beta_{j}} \frac{\delta_{i}}{1-p_{0 i}}+\sum_{i=1}^{n} \frac{\partial p_{0 i}}{\partial \beta_{j}} \frac{1-S_{\mathrm{W}}\left(z_{i}\right)}{p_{0 i}+S_{\mathrm{W}}\left(z_{i}\right)\left(1+p_{0 i}\right)}, æ=0, \cdots, p .
\end{aligned}
$$

O estimador de máxima verossimilhança (EMV) $\hat{\boldsymbol{\vartheta}}$ para o vetor de parâmetros $\boldsymbol{\vartheta}$ pode ser obtido por meio da solução do sistema de equações $U(\boldsymbol{\vartheta})=0$; como, geralmente, esse sistema é não linear, as soluções do sistema são obtidas numericamente usando algum método de otimização, 
tais como Newton-Raphson.

No caso em que o tamanho da amostra é grande, e sob certas condições de regularidade para a função de verossimilhança, o intervalo de confiança e o teste de hipóteses podem ser obtidos usando o fato de que a distribuição assintótica para os EMV é a distribuição normal com média $\vartheta$ e a matriz de covariância é dada pelo inverso da matriz de informação de Fisher. Portanto, a matriz de covariância assintótica é dada por $I^{-1}(\boldsymbol{\vartheta}) \operatorname{com} I(\boldsymbol{\vartheta})=E\left[-\left\{\frac{\partial^{2} \ell(\boldsymbol{\vartheta})}{\partial \boldsymbol{\vartheta} \partial \boldsymbol{\vartheta}^{\top}}\right\}\right]$.

Mas nas muitas situações a matriz de informação de Fisher não tem forma fechada e é dificilmente calculada; por isso, nesse caso, pode-se usar a negativa da matriz hessiana da função de $\log$-verossimilhança, denotada por $\ddot{L}(\boldsymbol{\vartheta})=-\left\{\frac{\partial^{2} \ell(\boldsymbol{\vartheta})}{\partial \boldsymbol{\vartheta} \partial \boldsymbol{\vartheta}^{\top}}\right\}$, avaliada em $\boldsymbol{\vartheta}=\hat{\boldsymbol{\vartheta}}$, que é um estimador consistente para matriz de covariância assintótica (Mudholkar et al., 1996). Assim, a distribuição assintótica para $\hat{\boldsymbol{\vartheta}}$ é dada por $\boldsymbol{\vartheta} \sim N_{(q)}\left\{\boldsymbol{\vartheta} ; \ddot{L}(\boldsymbol{\vartheta})^{-1}\right\}$ em que $q$ é o número de parâmetros do modelo e $\ddot{L}(\boldsymbol{\vartheta})$ é conhecido como a matriz de informação observada.

Considerando que uma partição $\boldsymbol{\vartheta}=\left(\boldsymbol{\vartheta}_{1}^{\top}, \boldsymbol{\vartheta}_{2}^{\top}\right)$, em que $\boldsymbol{\vartheta}_{1}$ é o subconjunto dos parâmetros de interesse e $\vartheta_{2}$ é o subconjunto dos parâmetros restantes. O teste de razão de verossimilhança $(\mathrm{RV})$ para testar a hipótese nula $H_{0}: \boldsymbol{\vartheta}_{1}=\boldsymbol{\vartheta}_{1}^{(0)}$ contra a hipótese alternativa $H_{1}$ : $\boldsymbol{\vartheta}_{1} \neq \boldsymbol{\vartheta}_{1}^{(0)}$ é dado por

$$
\omega=2\{\ell(\hat{\boldsymbol{\vartheta}})-\ell(\widetilde{\boldsymbol{\vartheta}})\}
$$

no qual $\hat{\boldsymbol{\vartheta}}$ e $\widetilde{\boldsymbol{\vartheta}}$ são os EMV sob as hipóteses nula e alternativa, respectivamente. Assim, a estatística $\omega$ é distribuída assintoticamente (quando $n \rightarrow \infty$ ) e tem a distribuição $\chi_{k}^{2}$, em que $k$ é a dimensão do subconjunto de interesse $\boldsymbol{\vartheta}_{1}$.

Alternativamente, os diferentes modelos podem ser comparados, penalizando os ajustes usando o critério de informação Akaike dado por $A I C=-2 \ell(\hat{\xi})+2 \#(\xi)$ e o critério de Schwartz-

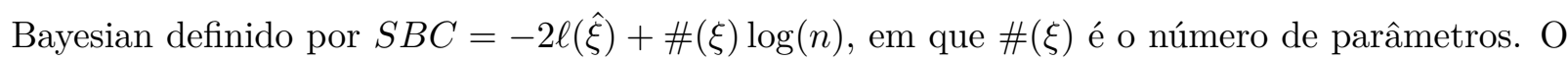
modelo com o menor valor de qualquer um desses critérios (entre todos os modos considerados) é geralmente considerado como o modelo mais adequado para descrever o conjunto de dados proposto.

\subsection{Estudo de simulação}

Um estudo de simulação foi realizado com o objetivo de comparar os modelos WBN com fração de cura na presença dos esquemas de primeira ativação (WBNcr-PA), última ativação 
(WBNcr-UA) e ativação aleatória (WBNcr-AA), e estudar o comportamento dos estimadores de máxima verossimilhança dos modelos propostos.

Nas simulações consideramos a covariável binária $x_{i}, i=1, \cdots, N$ foi gerado pela distribuição Bernoulli com parâmetro 0,5. Os tempos de ocorrência do evento de interesse $T_{i}$ são gerados a partir da distribuição Weibull (1.2) com o parâmetro $\alpha=2$ e $\lambda=-3$. Para cada indivíduo $i$, o número de causas do evento de interesse $\left(N_{i}\right)$ foi gerado pela distribuição Binomial Negativa (2.1) com parâmetro $\eta=2$ e $\left.\theta_{i}=\left(p_{0 i}^{-\eta}-1\right) / \eta\right)$ em que $p_{0 i}=\frac{\exp \left(\beta_{0}+\beta_{1} x_{i}\right)}{1+\exp \left(\beta_{0}+\beta_{1} x_{i}\right)}$. Fixamos os parâmetros $\beta_{0}=0,5$ e $\beta_{1}=-2$, e deste forma, as frações de cura para cada nível de covariável são dadas por $p_{0}^{(0)}=0,622$ e $p_{0}^{(1)}=0,182$, respectivamente. Os tempos de censura foram gerados a partir da distribuição uniforme $[0, \tau]$, na qual $\tau$ controla a proporção de censura. Também consideramos, aproximadamente, $50 \%$ de observações censuradas, sendo os tamanhos das amostras $N=50,100$ e 200, para cada configuração realizamos 1.000 replicas. O algoritmo para gerar dados dos tempos observados e indicadores de censura foi o seguinte:

1. Gerar $N_{i} \sim \operatorname{Binomial} \operatorname{Negativo}\left(\eta, \theta_{i}\right)$;

2. Se $N_{i}=0$, fazer $y_{i}=\infty$; caso contrário, gerar $T_{i} \sim$ Weibull $(\alpha, \lambda)$ para $i=1, \cdots, N_{i}$ e $y_{i}=R\left(T_{1}, \cdots, T_{N_{i}}\right)$;

3. Gerar $c_{i} \sim$ Uniforme $(0, \tau)$;

4. Considerar $z_{i}=\min \left\{y_{i}, c_{i}\right\}$;

5. Se $z_{i}<c_{i}$ então $\delta_{i}=1$, caso contrário, temos $\delta_{i}=0, i=1 \cdots, N$,

em que a função $R=$ Min, Max ou Random serve para gerar os tempos observados por meio da distribuição WBNcr sob esquema de primeira ativação, última e ativação aleatória, respectivamente.

Para cada conjunto de dados gerados, os três modelos (WBNcr-PA, WBNcr-UA e WBNcr-AA) foram ajustados e calculamos as estimativas de máxima verossimilhança (EMV) da fração de cura $\left(p_{0}^{(0)}\right.$ e $\left.p_{0}^{(1)}\right)$ para dois nível de covariável $\mathbf{x}$, os respectivos erros padrão (EP) e as raízes quadradas do erro quadrático médio(RQEM). Além disso, calculamos a porcentagem de amostra que foram geradas pela distribuição proposta e se que mostraram como o melhor modelo ajustado de acordo com o critério AIC. Ressaltamos que é possível discriminar entre as distribuições concorrentes pelo critério AIC.

A Tabela 4.2 apresenta as porcentagens das amostras que indicam o melhor modelo ajustado, e que de acordo com os critérios da AIC, a medida aumenta com o aumento do tamanho 
da amostra, mesmo que esta contenha uma porcentagem das observações censuradas. Além disso, o valor de RQEM do melhor modelo sempre é menor se comparado com as outras distribuições. Observamos, ainda que as médias das EMV da fração de cura do melhor modelo são próximas aos valores verdadeiros, e que os valores de EP e RQEM diminuem com o aumento do tamanho da amostra, conforme demostramos na Tabela 4.3.

TABELA 4.2: Porcentagem de amostras em que o modelo ajustado foi indicado como o melhor modelo de acordo com o critério AIC

\begin{tabular}{ccccc}
\hline Modelo & N & WBNcr-PA & WBNcr-UA & WBNcr-AA \\
\hline WBNcr-PA & 50 & 89,7 & 0,2 & 10,1 \\
& 100 & 98,9 & 0,0 & 1,1 \\
& 200 & 99,9 & 0,0 & 0,1 \\
\hline WBNcr-UA & 50 & 0,3 & 81,2 & 18,5 \\
& 100 & 0,0 & 97,3 & 2,7 \\
& 200 & 0,0 & 100,0 & 0,0 \\
\hline WBNcr-AA & 50 & 32,6 & 0,7 & 66,7 \\
& 100 & 22,2 & 1,7 & 76,1 \\
& 200 & 15,9 & 4,7 & 79,4 \\
\hline
\end{tabular}


TABELA 4.3: EMV, EP e RQEM das frações de cura $\left(p_{0}^{(0)}\right.$ e $\left.p_{0}^{(1)}\right)$ para os dados simulados

\begin{tabular}{|c|c|c|c|c|c|c|c|c|}
\hline \multirow[b]{2}{*}{ Modelo Gerado } & \multirow[b]{2}{*}{$\mathrm{N}$} & \multirow[b]{2}{*}{ Modelo Ajustado } & \multicolumn{3}{|c|}{$p_{0}^{(0)}$} & \multicolumn{3}{|c|}{$p_{0}^{(1)}$} \\
\hline & & & Média & EP & RQEM & Média & $\mathrm{EP}$ & RQEM \\
\hline \multirow{9}{*}{ WBNcr-PA } & 50 & WBNcr-PA & 0,616 & 0,105 & 0,105 & 0,170 & 0,070 & 0,071 \\
\hline & & WBNcr-UA & 0,591 & 0,131 & 0,135 & 0,226 & 0,073 & 0,085 \\
\hline & & WBNcr-AA & 0,627 & 0,108 & 0,108 & 0,162 & 0,074 & 0,077 \\
\hline & 100 & WBNcr-PA & 0,621 & 0,071 & 0,071 & 0,181 & 0,057 & 0,057 \\
\hline & & WBNcr-UA & 0,603 & 0,083 & 0,085 & 0,244 & 0,052 & 0,081 \\
\hline & & WBNcr-AA & 0,634 & 0,071 & 0,072 & 0,174 & 0,058 & 0,059 \\
\hline & 200 & WBNcr-PA & 0,622 & 0,050 & 0,050 & 0,183 & 0,043 & 0,043 \\
\hline & & WBNcr-UA & 0,605 & 0,057 & 0,060 & 0,250 & 0,039 & 0,078 \\
\hline & & WBNcr-AA & 0,634 & 0,050 & 0,051 & 0,175 & 0,045 & 0,045 \\
\hline \multirow[t]{9}{*}{ WBNcr-UA } & 50 & WBNcr-PA & 0,553 & 0,144 & 0,159 & 0,248 & 0,091 & 0,112 \\
\hline & & WBNcr-UA & 0,629 & 0,116 & 0,117 & 0,181 & 0,086 & 0,086 \\
\hline & & WBNcr-AA & 0,604 & 0,121 & 0,123 & 0,187 & 0,095 & 0,095 \\
\hline & 100 & WBNcr-PA & 0,562 & 0,092 & 0,110 & 0,270 & 0,064 & 0,108 \\
\hline & & WBNcr-UA & 0,625 & 0,077 & 0,077 & 0,191 & 0,067 & 0,067 \\
\hline & & WBNcr-AA & 0,605 & 0,079 & 0,081 & 0,198 & 0,071 & 0,072 \\
\hline & 200 & WBNcr-PA & 0,555 & 0,063 & 0,093 & 0,262 & 0,043 & 0,091 \\
\hline & & WBNcr-UA & 0,621 & 0,053 & 0,053 & 0,185 & 0,046 & 0,046 \\
\hline & & WBNcr-AA & 0,598 & 0,055 & 0,060 & 0,194 & 0,049 & 0,050 \\
\hline \multirow[t]{9}{*}{ WBNcr-AA } & 50 & WBNcr-PA & 0,634 & 0,110 & 0,110 & 0,234 & 0,096 & 0,109 \\
\hline & & WBNcr-UA & 0,642 & 0,111 & 0,113 & 0,239 & 0,094 & 0,109 \\
\hline & & WBNcr-AA & 0,648 & 0,105 & 0,108 & 0,212 & 0,102 & 0,106 \\
\hline & 100 & WBNcr-PA & 0,637 & 0,077 & 0,078 & 0,235 & 0,064 & 0,083 \\
\hline & & WBNcr-UA & 0,642 & 0,079 & 0,081 & 0,244 & 0,062 & 0,087 \\
\hline & & WBNcr-AA & 0,648 & 0,075 & 0,079 & 0,222 & 0,066 & 0,077 \\
\hline & 200 & WBNcr-PA & 0,645 & 0,051 & 0,056 & 0,236 & 0,046 & 0,071 \\
\hline & & WBNcr-UA & 0,648 & 0,053 & 0,059 & 0,250 & 0,043 & 0,080 \\
\hline & & WBNcr-AA & 0,654 & 0,050 & 0,059 & 0,226 & 0,047 & 0,064 \\
\hline
\end{tabular}

\subsection{Inferência Bayesiana}

Considere a situação em que os tempos de ocorrer o evento de interesse $y_{i}$ dados na Tabela 4.1 não são totalmente observados e estão sujeitos à censura à direita. Então, seja $z_{i}$ é o tempo observado tal que $z_{i}=\min \left\{y_{i}, c_{i}\right\}$, com $c_{i}$ sendo o tempo de censura e $\delta_{i}=I\left(y_{i} \leq c_{i}\right)$ sendo um indicador de censura, i.e., quando $\delta_{i}=1$ se $z_{i}$ for o tempo de falha e $\delta_{i}=0$ se ele é censurado à direita, para $i=1, \cdots, n$.

Seja $\mathbf{x}_{i}=\left(x_{i 1}, \cdots, x_{i p}\right)^{\top}$ o vetor de covariáveis para o $i$-ésimo indivíduo. Completando o nosso modelo, propomos para relacionar a fração de cura com as covariáveis, utilizando a ligação logística

$$
\log \left(\frac{p_{0 i}}{1-p_{0 i}}\right)=\mathbf{x}_{i}^{\top} \boldsymbol{\beta} \quad \text { ou } \quad p_{0 i}=\frac{\exp \left(\mathbf{x}_{i}^{\top} \boldsymbol{\beta}\right)}{1+\exp \left(\mathbf{x}_{i}^{\top} \boldsymbol{\beta}\right)}
$$


em que $\boldsymbol{\beta}=\left(\beta_{0}, \cdots, \beta_{p}\right)^{\top}$ denota o vetor dos coeficientes de regressão. Consideramos os dados com $n$ pares de tempo de evento e indicador de censura dado por $\left\{\left(z_{1}, \delta_{1}\right), \cdots,\left(z_{n}, \delta_{n}\right)\right\}$. A função de verossimilhança com censura não informativa é dada por

$$
L(\boldsymbol{\vartheta} ; \mathcal{D}) \propto \prod_{i=1}^{n} f_{\mathrm{pop}}\left(z_{i} ; \boldsymbol{\vartheta}\right)^{\delta_{i}} S_{\mathrm{pop}}\left(z_{i} ; \boldsymbol{\vartheta}\right)^{1-\delta_{i}}
$$

em que $\boldsymbol{\vartheta}=\left(\alpha, \lambda, \eta, \boldsymbol{\beta}^{\top}\right)^{\top}$ representa o vetor dos parâmetros e $\mathcal{D}=\left(n, \mathbf{Z}, \boldsymbol{\delta}, \mathbf{x}_{i}\right)$ são os dados observados, com $\mathbf{z}=\left(z_{1}, \ldots, z_{n}\right)^{\top}, \boldsymbol{\delta}=\left(\delta_{1}, \ldots, \delta_{n}\right)^{\top}$ e $\mathbf{x}_{i}=\left(x_{i 1}, \cdots, x_{i n}\right)^{\top}$, considerando que $f_{\text {pop }}(\cdot ; \boldsymbol{\vartheta})$ e $S_{\text {pop }}(\cdot ; \boldsymbol{\vartheta})$ são as funções de densidade e sobrevivência imprópria na Tabela 4.1.

$\mathrm{Na}$ análise Bayesiana, assumindo as seguintes densidades a priori para os parâmetros $\alpha$, $\lambda, \eta$ e $\boldsymbol{\beta}^{\top}$, temos:

- $\alpha \sim N\left(\mu_{\alpha}, \sigma_{\alpha}^{2}\right) I(\alpha>0), \mu_{\alpha}$ e $\sigma_{\alpha}$ conhecidos

- $\lambda \sim N\left(\mu_{\lambda}, \sigma_{\lambda}^{2}\right), \mu_{\lambda}$ e $\sigma_{\lambda}$ conhecidos

- $\eta \sim N\left(\mu_{\eta}, \sigma_{\eta}^{2}\right) I(\eta>0), \mu_{\eta}$ e $\sigma_{\eta}$ conhecidos

- $\beta_{j} \sim N\left(\mu_{0 j}, \sigma_{0 j}^{2}\right), \mu_{0 j}$ e $\sigma_{0 j}$ conhecidos, $j=0, \cdots, p$

Note que a escolha da distribuição de probabilidade Normal para as prioris, facilita a inserção de informação em uma determinada região de interesse através dos parâmetros $\mu$ e $\sigma^{2}$ da distribuição Normal $N\left(\mu, \sigma^{2}\right)$, mesmo que no caso de truncamento esses hiperparâmetros não representem a média e a variância, mas ainda controlam a região de maior massa de probabilidade. Neste caso, assumindo a independência dos parâmetros do modelo proposto, a função densidade a priori para $\vartheta$ pode ser escrita como:

$$
\pi(\boldsymbol{\vartheta})=\prod_{j=1}^{p} \pi\left(\beta_{j}\right) \pi(\alpha) \pi(\lambda) \pi(\eta)
$$

e para que as densidades a priori possuem informação vaga, consideramos que $\mu_{\alpha}=\mu_{\lambda}=\mu_{\eta}=$ $\mu_{0 j}=0, \sigma_{\alpha}^{2}, \sigma_{\lambda}^{2} \sigma_{\eta}^{2}$ e $\sigma_{0 j}^{2}$ assumem valores grandes.

Combinando a função de verossimilhança (4.21) e a função densidade a priori dada em (4.22), temos que a função densidade conjunta a posteriori para $\boldsymbol{\vartheta}$ é obtida por $\pi(\boldsymbol{\vartheta} \mid \mathcal{D}) \propto$ $L(\boldsymbol{\vartheta} ; \mathcal{D}) \prod_{j=1}^{p} \pi\left(\beta_{j}\right) \pi(\alpha) \pi(\lambda) \pi(\eta)$.

Como a função densidade conjunta a posteriori é difícil de ser tratada analiticamente, uma possível solução é utilizar os métodos de Monte Carlo via Cadeias de Markov (MCMC), 
tais como o Amostrador de Gibbs e o Metropolis-Hasting. Esses algoritmos podem ser utilizados para gerar amostras da distribuição a posteriori dos parâmetros $\pi(\boldsymbol{\vartheta} \mid \mathcal{D})$ através das distribuições condicionais completas $\pi\left(\vartheta_{i} \mid \boldsymbol{\vartheta}_{-i}, \mathcal{D}\right)$, sendo $\boldsymbol{\vartheta}_{-i}$ o vetor de todos os parâmetros exceto o parâmetro $\vartheta_{i}$. Como as distribuições condicionais completas dos modelos propostos não são identificáveis, o algoritmo Metropolis-Hastings foi utilizado nesse trabalho, tendo em vista que na literatura, é conhecido que a simulação MCMC na reta é mais eficiente; assim, utilizamos a transformação paramétrica $\boldsymbol{\varphi}=(\log (\alpha), \lambda, \log (\eta), \boldsymbol{\beta})=\left(\varphi_{1}, \lambda, \varphi_{2}, \boldsymbol{\beta}\right)$. Dessa forma, obtemos o espaço dos paramétrico $\mathbb{R}^{p+3}$ e a função densidade conjunta a posteriori, a qual é dada por

$$
\pi(\boldsymbol{\varphi} ; \mathcal{D}) \propto L(\boldsymbol{\varphi} ; \mathcal{D}) \exp \left\{-\frac{1}{2}\left[\sum_{j=1}^{p}\left(\frac{\beta_{0 j}^{2}}{\sigma_{0 j}}\right)^{2}+\frac{2 \exp \left(\varphi_{1}\right)}{\sigma_{\eta}{ }^{2}}+\frac{\lambda^{2}}{\sigma_{\lambda}{ }^{2}}+\frac{2 \exp \left(\varphi_{2}\right)}{\sigma_{\eta}{ }^{2}}\right]+\varphi_{1}+\varphi_{2}\right\}
$$

O algoritmo Metropolis-Hastings utilizado neste estudo foi demostrado na Seção 3.3 do Capítulo 2 .

\subsection{Estudo de dados artificiais}

Um estudo de simulação foi realizado com o objetivo de comparar o modelo de regressão proposto WBN com fração de cura com a presença dos três esquemas diferentes de ativação (modelo WBNcr-PA, WBNcr-UA, e WBNcr-AA).

Os critérios DIC, EAIC, EBIC, LPML foram considerados para a comparação de modelo, e as estimativas das frações de cura para cada nível da covariável, os respectivos erros padrão (EP) e as raízes quadradas do erro quadrático médio(RQEM) foram calculados utilizando as médias $a$ posteriori dos parâmetros dos modelos ajustados.

Na simulação, considerando a covariável binária $\mathbf{x}$, que foi gerada pela distribuição Bernoulli com parâmetros 0,5 , fixamos os valores dos parâmetros $\beta_{0}=0,5 ; \beta_{1}=-2 ; \eta=2$; $\alpha=2$ e $\lambda=-3$. Para cada modelo proposto foi gerada uma amostra de tamanho 600 , cujos tempos observados foram gerados usando o algoritmo dado na Seção 4.4. Para cada conjunto de dados gerado os três modelos propostos foram ajustados.

As densidades a priori consideradas para a simulação foram dadas na Seção 3.3, com $\mu_{\alpha}=\mu_{\lambda}=\mu_{\eta}=\mu_{0 j}=0, \sigma_{\alpha}^{2}=20^{2}, \sigma_{\lambda}^{2}=20^{2} \sigma_{\eta}^{2}=15^{2}$ e $\sigma_{0 j}^{2}=20^{2}$, e desta forma as densidades a priori possuem a informação vaga. Para cada conjunto de dados foi gerada uma cadeia de tamanho 50.000 e as primeiras 25.000 iterações foram desconsideradas para eliminar o efeito dos valores iniciais, assim obtivemos uma amostra efetiva de tamanho 25.000 sobre a qual a posteriori 
é baseada.

A Tabela 4.4 apresenta as estimativas dos critérios Bayesianos EAIC, EBIC, DIC e LPML para a seleção dos modelos. Note que, de acordo com esses critérios, para cada conjunto de dados simulados, o modelo correspondente apresentou o melhor ajuste. Portanto, é possível discriminar os três modelos propostos para um conjunto de dados. Além disso, as estimativas das frações de cura estão mais próximas dos valores teóricos $\left(p_{0}^{(0)}=0,6225\right.$ e $\left.p_{0}^{(1)}=0,1824\right)$ e os respectivos RQEM são sempre menores para o melhor modelo ajustado, o que pode ser observado na Tabela 4.5.

TABELA 4.4: Estimativas dos critérios Bayesianos dos modelos ajustados para os dados artificiais

\begin{tabular}{llcccc}
\hline \multirow{2}{*}{ Modelo Gerado } & Modelo Ajustado & Critério & & & \\
\cline { 3 - 6 } & & DIC & EAIC & EBIC & LPML \\
\hline \multirow{2}{*}{ WBNcr-PA } & WBNcr-PA & 1565,9739 & 1570,6486 & 1592,6333 & $-782,9186$ \\
& WBNcr-UA & 1679,3303 & 1685,3101 & 1707,2947 & $-839,6464$ \\
& WBNcr-AA & 1629,4235 & 1633,3490 & 1650,9367 & $-814,6584$ \\
\hline \multirow{2}{*}{ WBNcr-UA } & WBNcr-PA & 1886,1676 & 1892,3044 & 1914,2890 & $-943,1006$ \\
& WBNcr-UA & 1783,4519 & 1788,5755 & 1810,5602 & $-891,7152$ \\
& WBNcr-AA & 1853,8360 & 1857,8693 & 1875,4569 & $-926,8918$ \\
\hline \multirow{2}{*}{ WBNcr-AA } & WBNcr-PA & 1927,3480 & 1933,2535 & 1955,2381 & $-963,6487$ \\
& WBNcr-UA & 1927,0313 & 1932,9941 & 1954,9787 & $-963,4753$ \\
& WBNcr-AA & 1916,6736 & 1920,6282 & 1938,2159 & $-958,3198$ \\
\hline
\end{tabular}

TABELA 4.5: Estimativas das frações de cura, EP e RQEM para os dados artificiais

\begin{tabular}{llcccccc}
\hline \multirow{2}{*}{ Modelo Gerado } & \multicolumn{3}{c}{$p_{0}^{(0)}$} & \multicolumn{3}{c}{$p_{0}^{(1)}$} \\
\cline { 3 - 8 } WBNcr-PA & Modelo Ajustado & Média & EP & RQEM & Média & PE & RQEM \\
& WBNcr-PA & 0,6504 & 0,0287 & 0,0301 & 0,1924 & 0,0241 & 0,0260 \\
& WBNcr-AA & 0,6340 & 0,0300 & 0,0321 & 0,2490 & 0,0253 & 0,0712 \\
\hline WBNcr-UA & 0,6610 & 0,0287 & 0,0478 & 0,1842 & 0,0241 & 0,0242 \\
& WBNcr-PA & 0,5533 & 0,0338 & 0,0770 & 0,3306 & 0,0315 & 0,1515 \\
& WBNcr-UA & 0,6182 & 0,0299 & 0,0302 & 0,2325 & 0,0293 & 0,0581 \\
& WBNcr-AA & 0,5927 & 0,0322 & 0,0438 & 0,2520 & 0,0318 & 0,0765 \\
\hline WBNcr-AA & WBNcr-PA & 0,5600 & 0,0316 & 0,0700 & 0,1917 & 0,0244 & 0,0261 \\
& WBNcr-UA & 0,5771 & 0,0310 & 0,0549 & 0,1840 & 0,0237 & 0,0238 \\
& WBNcr-AA & 0,5809 & 0,0308 & 0,0518 & 0,1618 & 0,0240 & 0,0317 \\
\hline
\end{tabular}

\subsubsection{Análise de influência}

Um dos principais objetivos desse estudo é mostrar a necessidade dos modelos robustos para lidar com a presença da observação influente; assim, dois conjuntos de dados artificiais foram 
gerados a partir das distribuições WBNcr-PA, WBNcr-UA e WBNcr-AA, baseados nas mesmas configuração da Seção 4.6.

Selecionamos os casos 18 e 300 para perturbar a fim de criarmos observações influentes no conjunto de dados; além disso, escolhemos um ou dois casos e perturbamos a variável resposta da seguinte maneira $\widetilde{y_{i}}=y_{i}+4 S_{y}$ para i=18 e 300 , em que $S_{y}$ é o desvio padrão dos tempos $y_{i}$. Então, consideramos as seguintes configurações no estudo.

- Configuração A: o conjunto de dados sem perturbação;

- Configuração B: dados com o caso 18 perturbado;

- Configuração C: dados com o caso 300 perturbado;

- Configuração D: dados com os casos 18 e 300 perturbados.

O algoritmo MCMC é o mesmo da última seção, e a convergência do algorítimo M-H é monitorada pelo diagnóstico de convergência Geweke proposto por Geweke (1992). A Tabela 4.7 apresenta as estimativas das frações de cura $p_{0}^{(0)}$ e $p_{0}^{(1)}$, os EP e os RQEM que foram obtidos pelas médias a posteriori dos parâmetros dos modelos ajustados para os conjuntos de dados gerados.

As distribuições a posteriori são pouco sensíveis à perturbação dos casos selecionados, de fato as estimativas das frações de cura estão pouco afastadas dos valores teóricos $\left(p_{0}^{(0)}=0,6225\right.$ e $\left.p_{0}^{(0)}=0,1824\right)$, mas os respectivos EP e RQEM são maiores. A Tabela 3.4 apresentou as estimativas dos critérios bayesianos EAIC, EBIC, DIC e LPML para cada configuração considerada, na qual observamos que o modelo para os dados sem a perturbação apresenta um melhor ajuste. 
TABELA 4.6: Estimativas das frações de cura, EP e RQEM para os dados artificiais

\begin{tabular}{|c|c|c|c|c|c|c|c|c|}
\hline \multicolumn{9}{|l|}{$\begin{array}{c}\text { Modelo Gerado } \\
\text { WBNcr-PA }\end{array}$} \\
\hline Modelo & & Número & & $p_{0}^{(0)}$ & & & $p_{0}^{(1)}$ & \\
\hline Ajustado & Configuração & dos casos & Média & $\mathrm{EP}$ & RQEM & Média & $\mathrm{EP}$ & RQEM \\
\hline \multirow[t]{4}{*}{ WBNcr-PA } & $\mathrm{A}$ & nenhum & 0,6504 & 0,0287 & 0,0301 & 0,1924 & 0,0241 & 0,0260 \\
\hline & B & 18 & 0,5743 & 0,0311 & 0,0573 & 0,1971 & 0,0231 & 0,0274 \\
\hline & $\mathrm{C}$ & 300 & 0,6548 & 0,0299 & 0,0440 & 0,1479 & 0,0211 & 0,0404 \\
\hline & $\mathrm{D}$ & $\{18,300\}$ & 0,6528 & 0,0308 & 0,0432 & 0,1286 & 0,0198 & 0,0574 \\
\hline \multirow[t]{4}{*}{ WBNcr-UA } & A & nenhum & 0,6340 & 0,0300 & 0,0321 & 0,2490 & 0,0253 & 0,0712 \\
\hline & B & 18 & 0,5626 & 0,0310 & 0,0674 & 0,2611 & 0,0262 & 0,0829 \\
\hline & $\mathrm{C}$ & 300 & 0,6464 & 0,0303 & 0,0386 & 0,2039 & 0,0235 & 0,0318 \\
\hline & $\mathrm{D}$ & $\{18,300\}$ & 0,6646 & 0,0286 & 0,0509 & 0,2011 & 0,0244 & 0,0307 \\
\hline \multirow[t]{4}{*}{ WBNcr-AA } & $\bar{A}$ & nenhum & 0,6610 & 0,0287 & 0,0478 & 0,1842 & 0,0241 & 0,0242 \\
\hline & B & 18 & 0,5952 & 0,0295 & 0,0402 & 0,1970 & 0,0255 & 0,0293 \\
\hline & $\mathrm{C}$ & 300 & 0,6718 & 0,0220 & 0,0570 & 0,1538 & 0,0287 & 0,0361 \\
\hline & $\mathrm{D}$ & $\{18,300\}$ & 0,6881 & 0,0280 & 0,0714 & 0,1409 & 0,0226 & 0,0472 \\
\hline \multicolumn{9}{|l|}{$\begin{array}{l}\text { Modelo Gerado } \\
\text { WBNcr-UA }\end{array}$} \\
\hline \multirow[t]{4}{*}{ WBNcr-PA } & $\mathrm{A}$ & nenhum & 0,5533 & 0,0338 & 0,0770 & 0,3306 & 0,0315 & 0,1515 \\
\hline & B & 18 & 0,5284 & 0,2301 & 0,0298 & 0,0344 & 0,1002 & 0,0562 \\
\hline & $\mathrm{C}$ & 300 & 0,5632 & 0,0349 & 0,0688 & 0,2630 & 0,0316 & 0,0865 \\
\hline & $\mathrm{D}$ & $\{18,300\}$ & 0,6154 & 0,0343 & 0,0350 & 0,2160 & 0,0305 & 0,0454 \\
\hline \multirow[t]{4}{*}{ WBNcr-UA } & A & nenhum & 0,6182 & 0,0299 & 0,0302 & 0,2325 & 0,0293 & 0,0581 \\
\hline & B & 18 & 0,5768 & 0,0341 & 0,0570 & 0,1850 & 0,0293 & 0,0294 \\
\hline & $\mathrm{C}$ & 350 & 0,6251 & 0,0315 & 0,0316 & 0,2108 & 0,0296 & 0,0410 \\
\hline & $\mathrm{D}$ & $\{18,300\}$ & 0,6806 & 0,0292 & 0,0651 & 0,1467 & 0,0265 & 0,0445 \\
\hline \multirow[t]{4}{*}{ WBNcr-AA } & $\mathrm{A}$ & nenhum & 0,5927 & 0,0322 & 0,0438 & 0,2520 & 0,0318 & 0,0765 \\
\hline & B & 18 & 0,5793 & 0,0325 & 0,0540 & 0,1916 & 0,0285 & 0,0300 \\
\hline & $\mathrm{C}$ & 350 & 0,6116 & 0,0323 & 0,0341 & 0,2231 & 0,0300 & 0,0506 \\
\hline & $\mathrm{D}$ & $\{18,300\}$ & 0,6627 & 0,0307 & 0,0506 & 0,1700 & 0,0286 & 0,0312 \\
\hline \multicolumn{9}{|l|}{$\begin{array}{l}\text { Modelo Gerado } \\
\text { WBNcr-AA }\end{array}$} \\
\hline \multirow[t]{4}{*}{ WBNcr-PA } & A & nenhum & 0,5600 & 0,0316 & 0,0700 & 0,1917 & 0,0244 & 0,0261 \\
\hline & B & 18 & 0,6091 & 0,0307 & 0,0335 & 0,1668 & 0,0256 & 0,0300 \\
\hline & $\mathrm{C}$ & 300 & 0,5641 & 0,0323 & 0,0667 & 0,2069 & 0,0260 & 0,0357 \\
\hline & $\mathrm{D}$ & $\{18,300\}$ & 0,5329 & 0,0390 & 0,0977 & 0,1490 & 0,0242 & 0,0412 \\
\hline \multirow[t]{4}{*}{ WBNcr-UA } & $\mathrm{A}$ & nenhum & 0,5771 & 0,0310 & 0,0549 & 0,1840 & 0,0237 & 0,0238 \\
\hline & B & 18 & 0,6354 & 0,0296 & 0,0323 & 0,1566 & 0,0247 & 0,0357 \\
\hline & $\mathrm{C}$ & 300 & 0,5762 & 0,0327 & 0,0566 & 0,2145 & 0,0262 & 0,0414 \\
\hline & D & $\{18,300\}$ & 0,5754 & 0,0330 & 0,0575 & 0,1780 & 0,0245 & 0,0249 \\
\hline \multirow{4}{*}{ WBNcr-AA } & A & nenhum & 0,5809 & 0,0308 & 0,0518 & 0,1618 & 0,0240 & 0,0317 \\
\hline & B & 18 & 0,6382 & 0,0291 & 0,0331 & 0,1454 & 0,0254 & 0,0449 \\
\hline & $\mathrm{C}$ & 300 & 0,5863 & 0,0320 & 0,0483 & 0,1916 & 0,0263 & 0,0279 \\
\hline & D & $\{18,300\}$ & 0,5856 & 0,0328 & 0,0493 & 0,1603 & 0,0249 & 0,0333 \\
\hline
\end{tabular}


TABELA 4.7: Estimativas dos critérios Bayesianos dos modelos ajustados para os dados artificiais

\begin{tabular}{|c|c|c|c|c|c|c|c|c|c|c|c|c|c|}
\hline \multirow[b]{2}{*}{ Modelo Gerado } & \multirow[b]{2}{*}{ Configuração } & \multicolumn{4}{|c|}{ WBNcr-PA } & \multicolumn{4}{|c|}{ WBNcr-UA } & \multicolumn{4}{|c|}{ WBNcr-AA } \\
\hline & & DIC & EAIC & EBIC & LPML & DIC & EAIC & $\overline{\mathrm{EBIC}}$ & LPML & DIC & EAIC & EBIC & LPML \\
\hline \multirow[t]{4}{*}{ WBNcr-PA } & $\mathrm{A}$ & 1565,9 & 1570,6 & 1592,6 & $-782,9$ & 1679,3 & 1685,3 & 1707,2 & $-839,6$ & 1629,4 & 1633,3 & 1650,9 & $-814,6$ \\
\hline & B & 1785,2 & 1790,3 & 1812,3 & $-897,9$ & 1879,7 & 1885,7 & 1907,7 & $-942,2$ & 1840,7 & 1844,6 & 1862,1 & $-924,1:$ \\
\hline & C & 1695,6 & 1700,5 & 1722,5 & $-853,6$ & 1792,1 & 1798,0 & 1819,9 & $-899,1$ & 1746,4 & 1750,5 & 1768,1 & $-876,9=$ \\
\hline & D & 1661,2 & 1666,3 & 1688,3 & $-834,8$ & 1797,6 & 1803,7 & 1825,7 & $-901,1$ & 1744,4 & 1748,3 & 1765,9 & $-875,4^{2}$ \\
\hline \multirow[t]{4}{*}{ WBNcr-UA } & $\mathrm{A}$ & 1886,1 & 1892,3 & 1914,2 & $-943,1$ & 1783,4 & 1788,5 & 1810,5 & $-891,7$ & 1853,8 & 1857,8 & 1875,4 & $-926,8$ \\
\hline & B & 2036,1 & 2042,1 & 2064,1 & $-1033,0$ & 1963,5 & 1968,4 & 1990,4 & $-993,2$ & 2014,3 & 2018,4 & 2035,9 & $-1019,3$ \\
\hline & $\mathrm{C}$ & 1969,5 & 1975,5 & 1997,5 & $-995,1$ & 1899,3 & 1904,3 & 1926,3 & $-959,9$ & 1947,9 & 1952,1 & 1969,6 & $-984,1 \AA$ \\
\hline & $\mathrm{D}$ & 1915,2 & 1921,1 & 1943,1 & $-963,9$ & 1828,3 & 1833,3 & 1855,3 & $-921,2$ & 1886,4 & 1890,4 & 1908,0 & $-948,5$ \\
\hline \multirow[t]{4}{*}{ WBNcr-AA } & $\overline{\mathrm{A}}$ & $\begin{array}{l}1927,3 \\
\end{array}$ & $\begin{array}{l}1933,2 \\
\end{array}$ & 1955,2 & $-963,6$ & $\begin{array}{l}1927,0 \\
\end{array}$ & 1932,9 & 1954,9 & $-963,4$ & 1916,6 & 1920,6 & 1938,2 & $-958,3$ \\
\hline & B & 1988,1 & 1994,2 & 2016,2 & $-1006,1$ & 1970,0 & 1975,8 & 1997,8 & $-995,1$ & 1977,8 & 1981,7 & 1999,3 & $-998,8$ \\
\hline & $\mathrm{C}$ & 2000,3 & 2006,2 & 2028,2 & $-1005,9$ & 2004,4 & 2010,4 & 2032,4 & $-1006,1$ & 1998,3 & 2002,2 & 2019,7 & $-1005,0$ \\
\hline & $\mathrm{D}$ & 1927,3 & 1933,2 & 1955,2 & $-963,6$ & 1927,0 & 1932,9 & 1954,9 & $-963,4$ & 1916,6 & 1920,6 & 1938,2 & $-958,3$ \\
\hline
\end{tabular}


Também consideramos, neste trabalho, as amostras a posteriori dos parâmetros dos modelos WBNcr-PA, WBNcr-UA e WBNcr-AA, calculamos as estimativas de Monte Carlo das medidas de $\psi$-divergência descritas na Seção 1.4, com o objetivo de estudar as medidas consideradas na detecção das possíveis observações influentes na distribuição a posteriori dos modelos propostos.

As estimativas de Monte Carlo das medidas de $K$ - $L$ divergence, $L_{1}$-distance, $J$-distance e $\chi^{2}$-divergence são apresentadas na Tabela 4.8 e nas Figuras 4.2, 4.3 e 4.4, mostrando que as observações influentes foram detectadas em cada modelo de ajuste. Podemos ver claramente que todas as medidas tiveram um bom desempenho para identificar observações influentes e que, de fato, as estimativas de Monte Carlo das medidas de $\psi$-divergência dos casos perturbados apresentaram os valores maiores em relação aos outros casos.

TABELA 4.8: Estimativas das medidas de $\psi$-divergência para os dados artificiais.

\begin{tabular}{lcccccc}
\hline $\begin{array}{l}\text { Modelo } \\
\text { Gerado }\end{array}$ & Configuração & $\begin{array}{c}\text { Número } \\
\text { do caso }\end{array}$ & $d_{\mathrm{K}-\mathrm{L}}$ & $d_{\mathrm{J}}$ & $d_{L_{1}}$ & $d_{\chi^{2}}$ \\
\hline WBNcr-PA & $\mathrm{A}$ & 18 & 0,0006 & 0,0011 & 0,0130 & 0,0011 \\
& & 300 & 0,0009 & 0,0019 & 0,0178 & 0,0019 \\
& $\mathrm{~B}$ & 18 & 5,9070 & 10,9723 & 0,9262 & 523,8000 \\
& $\mathrm{C}$ & 300 & 6,4634 & 11,8767 & 0,9359 & 942,9992 \\
& $\mathrm{D}$ & 18 & 2,8595 & 5,8037 & 0,7865 & 115,4376 \\
& & 300 & 2,1468 & 4,3858 & 0,71949 & 44,8504 \\
\hline WBNcr-UA & $\mathrm{A}$ & 18 & 0,0023 & 0,0046 & 0,0272 & 0,0046 \\
& & 300 & 0,0018 & 0,0037 & 0,02457 & 0,0038 \\
& $\mathrm{~B}$ & 18 & 12,1768 & 19,0128 & 0,9803 & 2297,1616 \\
& $\mathrm{C}$ & 300 & 11,0372 & 17,7364 & 0,9788 & 2307,4451 \\
& $\mathrm{D}$ & 18 & 3,2048 & 7,09303 & 0,8179 & 464,3375 \\
& & 300 & 4,5338 & 9,7746 & 0,8928 & 1177,4437 \\
\hline WBNcr-AA & $\mathrm{A}$ & 18 & 0,0015 & 0,0029 & 0,02152 & 0,0029 \\
& & 300 & 0,0046 & 0,0093 & 0,03875 & 0,0095 \\
& $\mathrm{~B}$ & 18 & 10,5204 & 16,8135 & 0,9746 & 1223,7157 \\
& $\mathrm{C}$ & 300 & 6,3472 & 12,5478 & 0,9401 & 2008,1666 \\
& $\mathrm{D}$ & 18 & 5,40941 & 11,5296 & 0,9277 & 2120,5324 \\
& & 300 & 6,0425 & 12,5588 & 0,9427 & 2567,6781 \\
\hline
\end{tabular}



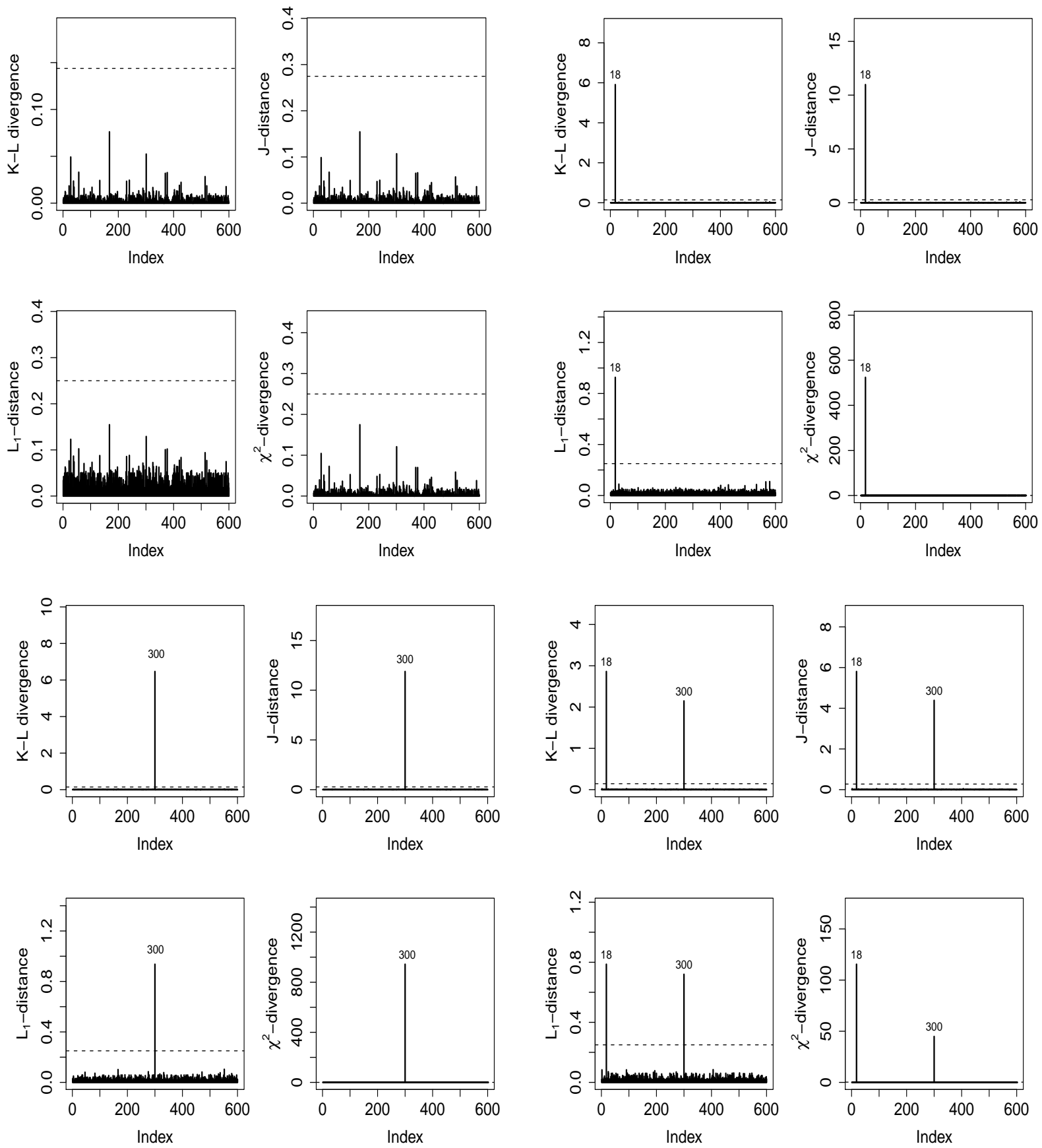

FIGURA 4.2: Estimativas de Monte Carlo das medidas de $\psi$-divergência do modelo de regressão WBNcr-PA ajustado. Painel superior esquerdo: Configuração A, conjunto de dados sem perturbação. Painel superior direito: Configuração B, conjunto de dados com observação 18 perturbada. Painel inferior esquerdo: Configuração C, conjunto de dados com observação 300 perturbada. Painel inferior direito: Configuração D, conjunto de dados com observações 18 e 300 perturbadas. 

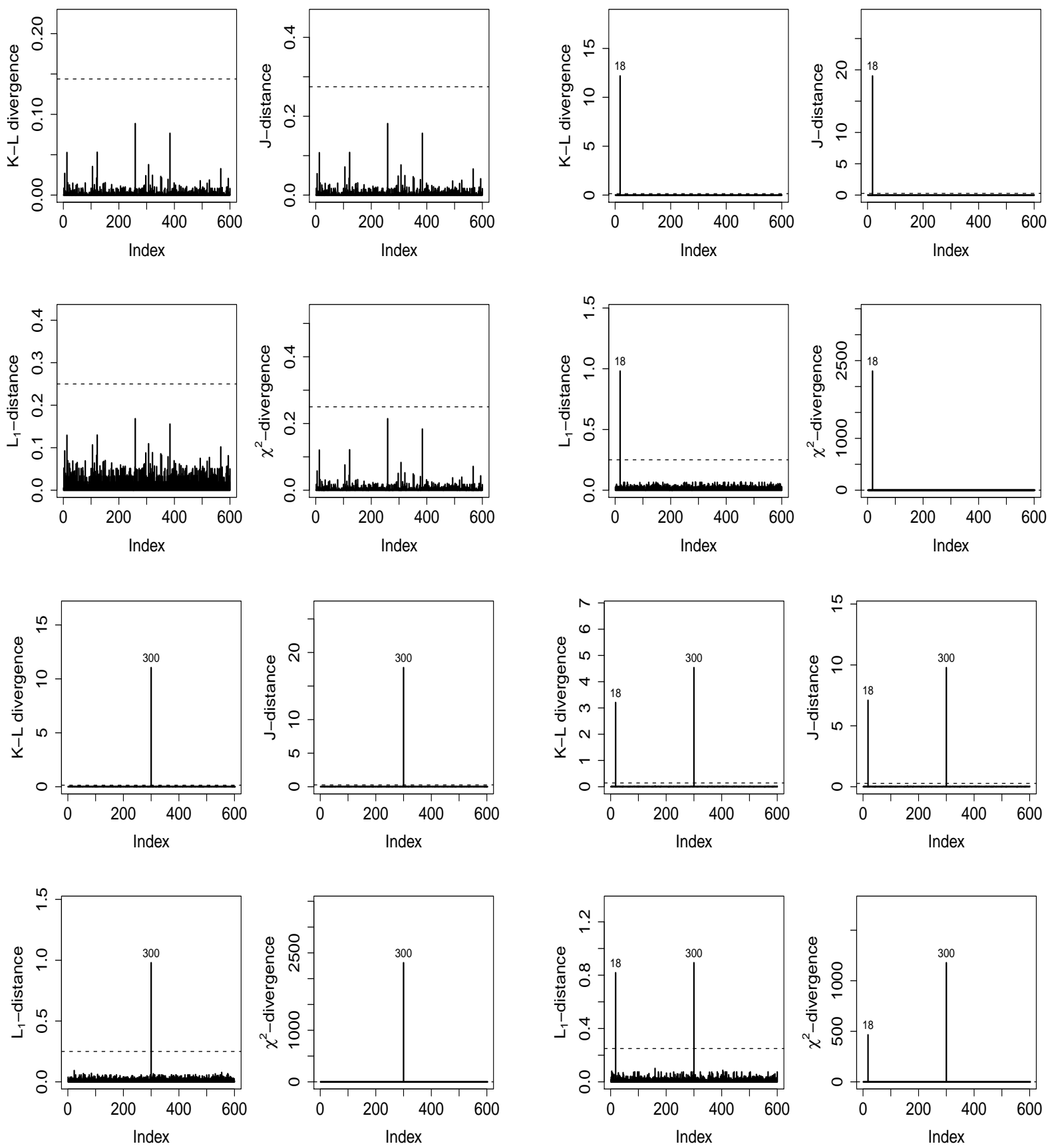

FIGURA 4.3: Estimativas de Monte Carlo das medidas de $\psi$-divergência do modelo de regressão WBNcr-UA ajustado. Painel superior esquerdo: Configuração A, conjunto de dados sem perturbação. Painel superior direito: Configuração B, conjunto de dados com observação 18 perturbada. Painel inferior esquerdo: Configuração C, conjunto de dados com observação 300 perturbada. Painel inferior direito: Configuração D, conjunto de dados com observações 18 e 300 perturbadas. 

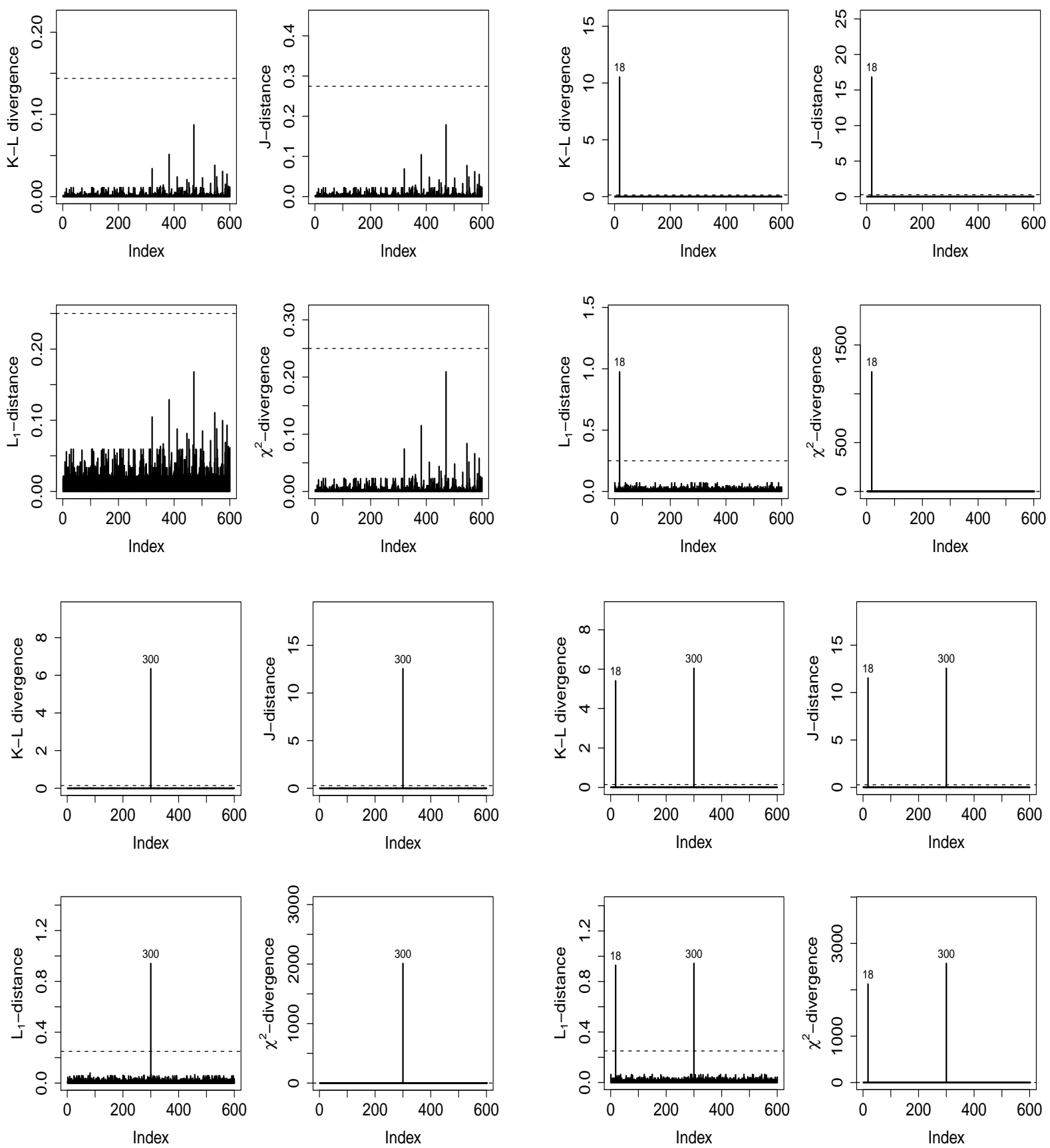

FIGURA 4.4: Estimativas de Monte Carlo das medidas de $\psi$-divergência do modelo de regressão WBNcr-AA ajustado. Painel superior esquerdo: Configuração A, conjunto de dados sem perturbação. Painel superior direito: Configuração B, conjunto de dados com observação 18 perturbada. Painel inferior esquerdo: Configuração C, conjunto de dados com observação 300 perturbada. Painel inferior direito: Configuração D, conjunto de dados com observações 18 e 300 perturbadas. 


\subsection{Aplicação}

Os dados considerados nesta seção provém de um estudo de melanoma com o objetivo de avaliar a eficácia da aplicação de uma dosagem alta de interferon alfa-2b como forma de prevenir a recorrência de câncer. Os dados foram obtidos entre 1991 e 1995, mas houve acompanhamento dos pacientes até 1998. A variável resposta representa o tempo até a morte dos paciente ou o tempo de censura. Mais detalhes deste conjunto de dados podem ser encontrados em Ibrahim et al. (2001). Da amostra original foram desconsiderados 10 pacientes devido à presença de dados faltantes, resultando $n=417$ pacientes, com $56 \%$ de observações censuradas. As variáveis consideradas neste estudo incluem $t$ : tempo observado (em anos, média $=3,179$ e desvio padrão=1,692); $x_{1 i}$ : tipo de tratamento (0: observação, $n=204 ; 1$ : interferon, $n=213$ ); $x_{2 i}$ : idade (em anos, média $=48,000$ e desvio padrão=13,121); $x_{3 i}$ : categoria do nódulo $(1, n=111 ; 2, n=137 ; 3$, $n=87 ; 4, n=82), i=1, \cdots, 417$ e $x_{4 i}$ : sexo dos pacientes (0: masculino $n=263 ; 1$ :feminino $n=154) ; x_{5 i}$ : capacidade funcional (0: ativo, $n=363 ; 1$ : outras, $\left.n=54\right)$ e $x_{6 i}$ espessura do tumor (em $m m$, média=3,941 e desvio padrão 3,204).

$\mathrm{Na}$ aplicação, fizemos o estudo sob as abordagens clássica e Bayesiana, considerando o modelo de regressão WBN com fração de cura sobre o esquema de primeira, última ativação e ativação aleatória, e relacionado a fração de cura $p_{0}$ com todas as covariáveis, utilizando a função de ligação logística com todas covariáveis, dada por:

$$
\log \left(\frac{p_{0 i}}{1-p_{0 i}}\right)=\beta_{0}+\beta_{1} x_{i 1}+\beta_{2} x_{i 2}+\beta_{3} x_{i 3}+\beta_{4} x_{i 4}+\beta_{5} x_{i 5}+\beta_{6} x_{i 6}
$$

\subsubsection{Análise Clássica}

Primeiramente, os modelos propostos (WBNcr-PA, WBNcr-UA e WBNcr-AA) e seus sub-modelos (WGcr-PA, WGcr-UA e WGcr-AA) foram ajustados para o conjunto de dados. Na Tabela 4.9 apresentamos as estimativas dos critérios de seleção dos modelos candidatos, e de acordo com os critérios AIC e SBC, o modelo WBNcr-PA se destaca como o modelo mais adequado. Na esquerda da Figura 4.5 temos as estimativas de Kaplan-Meier da função de sobrevivência para os dados descritos anteriormente, em que a curva estabilizou em 0,48, indicando a existência de uma apreciável fração de indivíduos curados. 

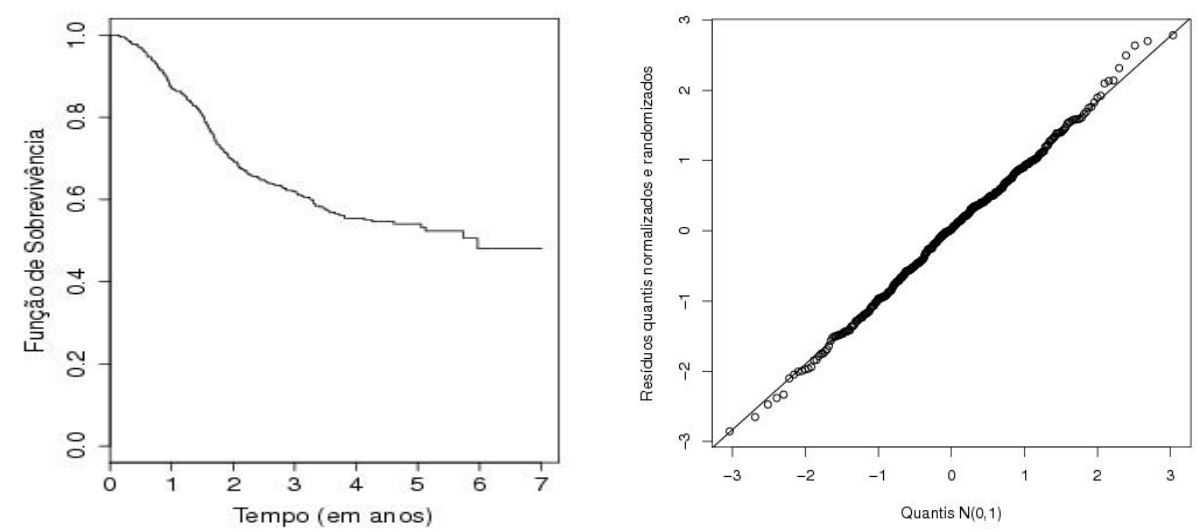

FIGURA 4.5: Painel esquerdo: Estimativas de Kaplan-Meier da função de sobrevivência. Painel direito: Gráfico QQ dos resíduos quantis aleatorizados e normalizados do modelo ajustado WBNcr-PA

O gráfico QQ dos resíduos quantis aleatorizados e normalizados (Dunn \& Smyth, 1996) à direita na Figura 4.5 sugere que o modelo WBNcr-PA produz um ajuste adequado. Considerando os critérios estabelecidos na Tabela 4.9 e o gráfico QQ mostrado na Figura 4.5, selecionamos o modelo WBNcr-PA como nosso modelo de trabalho.

TABELA 4.9: Critérios AIC e SBC para os modelos ajustados

\begin{tabular}{lcccccc}
\hline Model & WBNcr-PA & WBNcr-UA & WBNcr-AA & WGcr-PA & WGcr-UA & WGcr-AA \\
\hline AIC & 1030,0 & 1039,5 & 1116,1 & 1032,8 & 1063,8 & 1116,1 \\
SBC & 1070,3 & 1079,8 & 1152,4 & 1069,1 & 1100,1 & 1152,4 \\
\hline
\end{tabular}

TABELA 4.10: Estimativas dos EMV dos parâmetros para o modelo WBNcr-PA

\begin{tabular}{lcccc}
\hline Parâmetros & Estimativa (EST) & Erro Padrão (EP) & $\mid$ EST $\mid / \mathrm{EP}$ & p-valor \\
\hline$\alpha$ & 2,311 & 0,209 & 11,056 & - \\
$\lambda$ & $-3,113$ & 1,191 & 2,613 & - \\
$\eta$ & 3,390 & 0,748 & 4,532 & - \\
$\beta_{\text {intercepto }}$ & 1,354 & 0,664 & 2,040 & 0,041 \\
$\beta_{\text {tratamento }}$ & 0,140 & 0,184 & 0,763 & 0,445 \\
$\beta_{\text {idade }}$ & $-0,010$ & 0,007 & 1,483 & 0,138 \\
$\beta_{\text {nódulo }}$ & $-0,395$ & 0,136 & 2,915 & 0,004 \\
$\beta_{\text {sexo }}$ & 0,034 & 0,204 & 0,165 & 0,869 \\
$\beta_{\text {capacidade }}$ & $-0,139$ & 0,215 & 0,649 & 0,517 \\
$\beta_{\text {espessura }}$ & $-0,007$ & 0,027 & 0,269 & 0,788 \\
\hline
\end{tabular}

Considerando o nível de significância usual 5\%, observamos que todas as covariáveis são não significativas, com exceção da covariável de categoria do nódulo $\left(x_{3}\right)$ na Tabela 4.10. Assim, 
ajustamos o modelo WBNcr-PA sem covariáveis não significativas. Os EMV e seus erros padrão dos parâmetros do modelo ajustado são dados na Tabela 4.11.

TABELA 4.11: Estimativas dos EMV dos parâmetros para o modelo WBNcr-PA

\begin{tabular}{lcccc}
\hline Parâmetros & Estimativa (EST) & Erro Padrão (EP) & $\mid$ EST $\mid /$ EP & p-valor \\
\hline$\alpha$ & 2.2791 & 0,1211 & 6,801 & - \\
$\lambda$ & $-3,0490$ & 0,6595 & $-4,623$ & - \\
$\eta$ & 3.2808 & 0,4073 & 2,9169 & 0,0037 \\
$\beta_{\text {intercepto }}$ & 0,9082 & 0,2745 & 3,309 & $<0,001$ \\
$\beta_{\text {nódulo }}$ & $-0,3906$ & 0,0925 & $-4,222$ & $<0,001$ \\
\hline
\end{tabular}

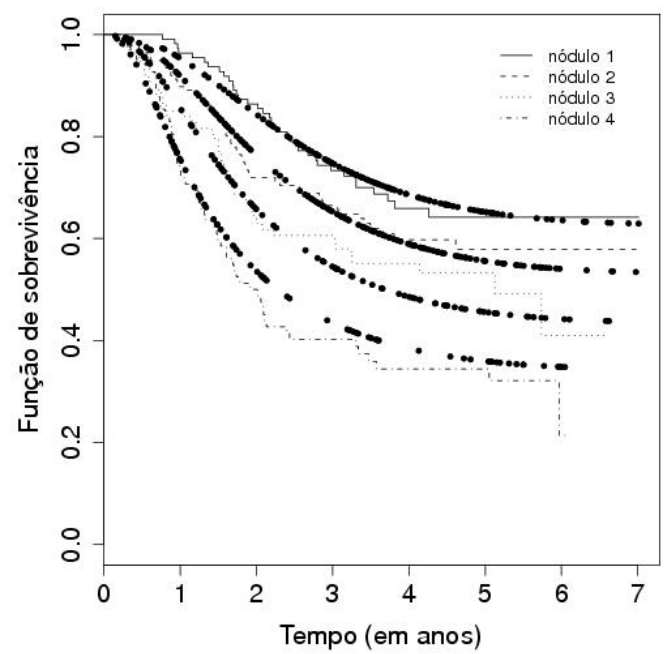

FIGURA 4.6: Estimativas de Kaplan-Meier e paramétricas da função de sobrevivência de acordo com a categoria nódulo (1 a 4 ).

TABELA 4.12: Resumo da fração de cura estratificada por categoria do nódulo.

\begin{tabular}{ccccc}
\hline Fração de & & & \multicolumn{2}{c}{ Percentil } \\
Cura & Média & Desvio padrão & $2,5 \%$ & $97,5 \%$ \\
\hline$p_{0_{1}}$ & 0,6266 & 0,0301 & 0,2864 & 0,9668 \\
$p_{0_{2}}$ & 0,5317 & 0,0208 & 0,2491 & 0,8143 \\
$p_{0_{3}}$ & 0,4345 & 0,0184 & 0,1686 & 0,7004 \\
$p_{0_{4}}$ & 0,3420 & 0,0252 & 0,0312 & 0,6529 \\
\hline
\end{tabular}

A Figura 4.6 mostrou que o modelo fornece uma boa aproximação das estimativas de Kaplan-Meier da função de sobrevivência. Na Tabela 4.11, a covariável de categoria do nódulo é significativa, pois, há uma relação com a classificação quanto ao tumor; desse modo, percebemos que a fração de cura diminui em indivíduos com maior categoria de nódulo. 


\subsubsection{Análise Bayesiana}

Assim como na abordagem clássica, primeiramente ajustamos os dados com os modelos de regressão WBNcr-PA, WBNcr-UA e WBNcr-AA para a sua comparação. De acordo com os valores das critérios DIC, EAIC, EBIC e LPML na Tabela 4.13, o modelo de regressão WBNcr-PA se apresenta como o melhor modelo ajustado, e por isso foi selecionado como o modelo de trabalho. O resumo a posteriori dos parâmetros do modelo de WBNcr-PA é apresentado na Tabela 4.14. Note que todas as covariáveis não são significativas exceto a covariável de categoria de nódulo $\left(\mathbf{x}_{3}\right)$.

TABELA 4.13: Conjunto de dados reais. Critérios Bayesianos para modelos selecionados.

\begin{tabular}{lccc}
\hline & \multicolumn{3}{c}{ Ativação } \\
\cline { 2 - 4 } Critério & Primeira & Última & Aleatória \\
\hline DIC & 1029,8337 & 1057,2525 & 1101,6494 \\
EAIC & 1039,9938 & 1067,9487 & 1117,87934 \\
EBIC & 1080,3247 & 1108,2796 & 1154,1771 \\
LPML & $-515,8537$ & $-528,95439$ & $-550,7612$ \\
\hline
\end{tabular}

TABELA 4.14: Conjunto de dados reais. Resumo a posteriori dos parâmetros para o modelo de regressão WBNcr-PA.

\begin{tabular}{clccccc}
\hline \multirow{2}{*}{ Dados } & Parâmetro & Média & Mediana & EP & LI & LS \\
\hline Original & $\alpha$ & 2,443 & 2,363 & 0,442 & 1,770 & 3,422 \\
& $\lambda$ & $-3,548$ & $-3,296$ & 1,056 & $-5,780$ & $-2,038$ \\
& $\eta$ & 3,995 & 3,564 & 2,059 & 0,690 & 8,085 \\
& $\beta_{0}$ & 1,255 & 1,225 & 0,519 & 0,319 & 2,296 \\
& $\beta_{1}$ & 0,140 & 0,155 & 0,167 & $-0,177$ & 0,462 \\
& $\beta_{2}$ & $-0,009$ & $-0,008$ & 0,005 & $-0,020$ & 0,001 \\
& $\beta_{3}$ & $-0,387$ & $-0,380$ & 0,100 & $-0,573$ & $-0,188$ \\
& $\beta_{4}$ & 0,041 & 0,029 & 0,177 & $-0,291$ & 0,391 \\
& $\beta_{5}$ & $-0,153$ & $-0,144$ & 0,216 & $-0,576$ & 0,272 \\
& $\beta_{6}$ & $-0,007$ & $-0,006$ & 0,024 & $-0,055$ & 0,040 \\
\hline
\end{tabular}

Para detectar as possíveis observações influentes na distribuição a posteriori dos parâmetros do modelo, as estimativas das medidas de $\psi$-divergência foram obtidas pela amostra $a$ posteriori dos parâmetros do modelo. O painel direito da Figura 4.7 mostrou as estimativas de Monte Carlo das medidas de $K-L$ divergence, $L_{1}$-distance, J-distance e $\chi^{2}$-divergence, onde as observações 23, 176 e 279 foram indicadas como possíveis observações influentes. Na tabela 4.15 apresentamos as informações dos casos detectados, em que o estado é um indicador de censura, 
de modo que se estado $=1$, indica que o evento ocorre e se estado $=0$, ele é censura à direita. Observamos que os tempos dos casos detectados apresentam os valores mínimos e máximos do conjunto de dados e o sexo deles é masculino.
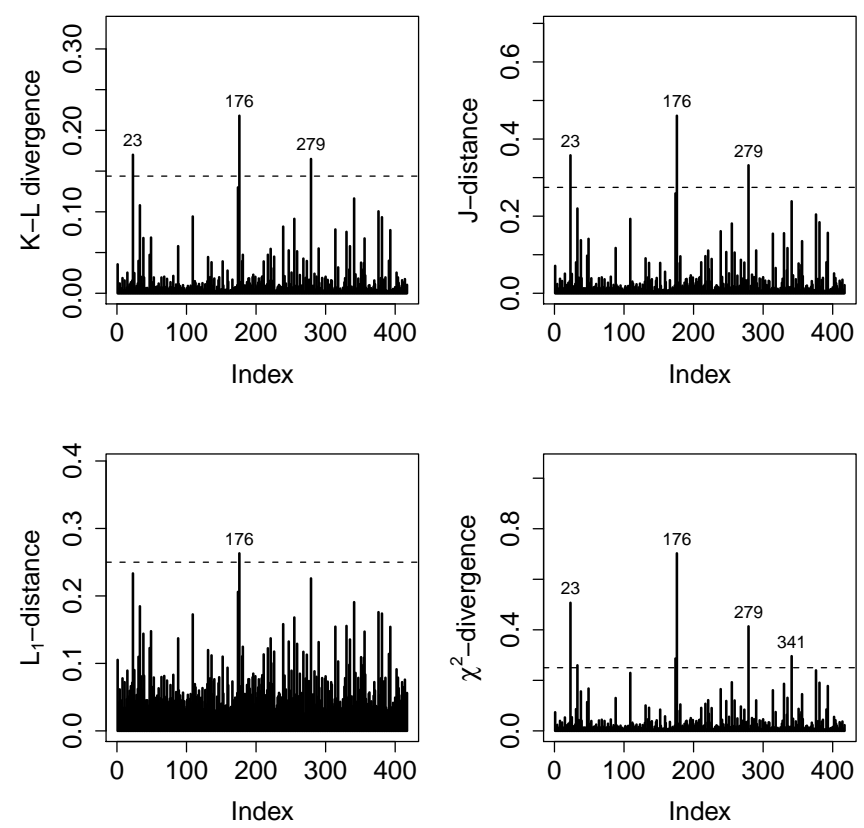

FIGURA 4.7: Estimativas de Monte Carlo das medidas de $\psi$-divergência do modelo de regressão WBNcr-PA ajustado para conjunto de dados original

TABELA 4.15: Possíveis observações influentes detectadas por $\psi$-divergence

\begin{tabular}{lccccccccccc}
\hline $\begin{array}{l}\text { Observa- } \\
\text { ções }\end{array}$ & $\begin{array}{c}\text { tempo } \\
\text { (estado) }\end{array}$ & $\begin{array}{c}\text { trata- } \\
\text { mento }\end{array}$ & idade & $\begin{array}{c}\text { categoria de } \\
\text { nódulo }\end{array}$ & sexo & $\begin{array}{c}\text { capacidade } \\
\text { functional }\end{array}$ & espessura & $d_{\mathrm{K}-\mathrm{L}}$ & $d_{\mathrm{J}}$ & $d_{L_{1}}$ & $d_{\chi^{2}}$ \\
\hline 23 & $5.74(1)$ & 1 & 36 & 3 & 0 & 0 & 2.04 & 0.170 & 0.358 & 0.233 & 0.507 \\
176 & $5.97(1)$ & 0 & 53 & 4 & 0 & 0 & 1.92 & 0.218 & 0.460 & 0.263 & 0.703 \\
279 & $0.17(1)$ & 0 & 68 & 4 & 0 & 1 & 3.00 & 0.165 & 0.332 & 0.226 & 0.414 \\
\hline
\end{tabular}

Com o objetivo de estudar o impacto dessas possíveis observações influentes na estimativa do parâmetro e na análise de inferência, reajustamos o modelo de regressão WBNcr-PA sobre as seguinte situações: primeiramente, cada um dos casos detectados foi eliminado individualmente. Depois, removemos dois a dois dos casos para todas possíveis combinações; no final, todos casos influentes foram eliminados do conjunto de dados originais, assim, o resumo a posteriori dos parâmetros do modelo de regressão WBNcr-PA reajustado é apresentado na Tabela 4.16. Ressaltamos as médias a posteriori dos parâmetros do modelo de regressão WBNcr-PA não são muito sensíveis à eliminação das observações influentes; observamos que somente as médias $a$ posteriori dos parâmetros $\lambda$ e $\eta$ mudaram quando o modelo foi ajustado para os dados sem os observações 23, 176 e 279. Além disso, os resultados de todas possíveis combinações mostram somente a covariável de categoria de nódulo $\left(\mathbf{x}_{3}\right)$ é significativa. 
TABELA 4.16: Resumo a posteriori dos parâmetros do modelo de regressão WBNcr-PA para dados reais

\begin{tabular}{|c|c|c|c|c|c|c|}
\hline \multirow[b]{2}{*}{ Dados } & \multirow[b]{2}{*}{ Parâmetro } & \multirow[b]{2}{*}{ Média } & \multirow[b]{2}{*}{ Mediana } & \multirow[b]{2}{*}{$\mathrm{EP}$} & \multicolumn{2}{|c|}{ Intervalo HPD (95\%) } \\
\hline & & & & & LI & $\mathrm{LS}$ \\
\hline Sem & $\alpha$ & 2,366 & 2,299 & 0,378 & 1,738 & 3,151 \\
\hline Observação & $\lambda$ & $-3,100$ & $-2,941$ & 0,844 & $-4,808$ & $-1,733$ \\
\hline \multirow[t]{8}{*}{23} & $\eta$ & 3,371 & 3,0300 & 1,880 & 0,310 & 7,027 \\
\hline & $\beta_{0}$ & 1,466 & 1,423 & 0,527 & 0,431 & 2,430 \\
\hline & $\beta_{1}$ & 0,110 & 0,126 & 0,182 & $-0,269$ & 0,440 \\
\hline & $\beta_{2}$ & $-0,010$ & $-0,009$ & 0,006 & $-0,023$ & 0,000 \\
\hline & $\beta_{3}$ & $-0,420$ & $-0,412$ & 0,104 & $-0,626$ & $-0,235$ \\
\hline & $\beta_{4}$ & 0,066 & 0,052 & 0,180 & $-0,275$ & 0,429 \\
\hline & $\beta_{5}$ & $-0,143$ & $-0,140$ & 0,226 & $-0,586$ & 0,313 \\
\hline & $\beta_{6}$ & $-0,010$ & $-0,008$ & 0,025 & $-0,062$ & 0,040 \\
\hline Sem & $\alpha$ & 2,428 & 2,345 & 0,424 & 1,752 & 3,297 \\
\hline Observação & $\lambda$ & $-3,287$ & $-3,0033$ & 1,0916 & $-6,178$ & $-1,924$ \\
\hline \multirow[t]{8}{*}{176} & $\eta$ & 3,643 & 3,207 & 2,063 & 0,518 & 7,721 \\
\hline & $\beta_{0}$ & 1,417 & 1,409 & 0,524 & 0,471 & 2,503 \\
\hline & $\beta_{1}$ & 0,139 & 0,154 & 0,166 & $-0,209$ & 0,436 \\
\hline & $\beta_{2}$ & $-0,010$ & $-0,009$ & 0,006 & $-0,022$ & 0,001 \\
\hline & $\beta_{3}$ & $-0,415$ & $-0,412$ & 0,103 & $-0,614$ & $-0,226$ \\
\hline & $\beta_{4}$ & 0,054 & 0,044 & 0,186 & $-0,296$ & 0,410 \\
\hline & $\beta_{5}$ & $-0,132$ & $-0,140$ & 0,214 & $-0,564$ & 0,296 \\
\hline & $\beta_{6}$ & $-0,007$ & $-0,006$ & 0,025 & $-0,059$ & 0,041 \\
\hline Sem & $\alpha$ & 2,421 & 2,306 & 0,473 & 1,818 & 3,566 \\
\hline Observação & $\lambda$ & $-3,325$ & $-3,058$ & 1,071 & $-5,438$ & $-1,741$ \\
\hline \multirow[t]{8}{*}{279} & $\eta$ & 3,685 & 3,108 & 2,347 & 0,524 & 9,141 \\
\hline & $\beta_{0}$ & 1,358 & 1,343 & 0,577 & 0,390 & 2,488 \\
\hline & $\beta_{1}$ & 0,112 & 0,135 & 0,185 & $-0,241$ & 0,479 \\
\hline & $\beta_{2}$ & $-0,009$ & $-0,009$ & 0,007 & $-0,024$ & 0,003 \\
\hline & $\beta_{3}$ & $-0,399$ & $-0,392$ & 0,118 & $-0,656$ & $-0,207$ \\
\hline & $\beta_{4}$ & 0,039 & 0,021 & 0,198 & $-0,282$ & 0,448 \\
\hline & $\beta_{5}$ & $-0,079$ & $-0,059$ & 0,227 & $-0,542$ & 0,379 \\
\hline & $\beta_{6}$ & $-0,009$ & $-0,007$ & 0,026 & $-0,065$ & 0,039 \\
\hline Sem & $\alpha$ & 2,428 & 2,349 & 0,406 & 1,737 & 3,348 \\
\hline Observações & $\lambda$ & $-2,989$ & $-2,796$ & 0,845 & $-4,851$ & $-1,651$ \\
\hline \multirow[t]{8}{*}{23 e 176} & $\eta$ & 3,309 & 2,858 & 1,953 & 0,375 & 7,587 \\
\hline & $\beta_{0}$ & 1,538 & 1,508 & 0,514 & 0,575 & 2,550 \\
\hline & $\beta_{1}$ & 0,126 & 0,135 & 0,185 & $-0,242$ & 0,466 \\
\hline & $\beta_{2}$ & $-0,010$ & $-0,009$ & 0,006 & $-0,022$ & 0,001 \\
\hline & $\beta_{3}$ & $-0,435$ & $-0,431$ & 0,107 & $-0,641$ & $-0,237$ \\
\hline & $\beta_{4}$ & 0,064 & 0,049 & 0,191 & $-0,287$ & 0,458 \\
\hline & $\beta_{5}$ & $-0,134$ & $-0,129$ & 0,232 & $-0,588$ & 0,332 \\
\hline & $\beta_{6}$ & $-0,008$ & $-0,007$ & 0,027 & $-0,066$ & 0,039 \\
\hline Sem & $\alpha$ & 2,362 & 2,299 & 0,352 & 1,845 & 3,196 \\
\hline Observações & $\lambda$ & $-3,047$ & $-2,866$ & 0,792 & $-4,658$ & $-1,773$ \\
\hline 23 and 279 & $\eta$ & 3,247 & 2,825 & 1,746 & 0,7670 & 7,090 \\
\hline & $\beta_{0}$ & 1,434 & 1,399 & 0,534 & 0,482 & 2,523 \\
\hline & $\beta_{1}$ & 0,107 & 0,112 & 0,178 & $-0,274$ & 0,422 \\
\hline & $\beta_{2}$ & $-0,009$ & $-0,008$ & 0,006 & $-0,022$ & 0,002 \\
\hline & $\beta_{3}$ & $-0,421$ & $-0,417$ & 0,105 & $-0,629$ & $-0,229$ \\
\hline & $\beta_{4}$ & 0,052 & 0,043 & 0,184 & $-0,274$ & 0,434 \\
\hline & $\beta_{5}$ & $-0,077$ & $-0,070$ & 0,234 & $-0,570$ & 0,346 \\
\hline & $\beta_{6}$ & $-0,008$ & $-0,008$ & 0,026 & $-0,061$ & 0,042 \\
\hline Sem & $\alpha$ & 2,350 & 2,309 & 0,325 & 1,752 & 2,994 \\
\hline Observações & $\lambda$ & $-2,983$ & $-2,855$ & 0,694 & $-4,436$ & $-1,806$ \\
\hline 176 e 279 & $\eta$ & 3,086 & 2,828 & 1,536 & 0,531 & 6,156 \\
\hline & $\beta_{0}$ & 1,448 & 1,429 & 0,517 & 0,448 & 2,477 \\
\hline & $\beta_{1}$ & 0,111 & 0,119 & 0,175 & $-0,238$ & 0,458 \\
\hline & $\beta_{2}$ & $-0,009$ & $-0,009$ & 0,006 & $-0,023$ & 0,002 \\
\hline & $\beta_{3}$ & $-0,427$ & $-0,423$ & 0,104 & $-0,639$ & $-0,236$ \\
\hline & $\beta_{4}$ & 0,061 & 0,052 & 0,187 & $-0,295$ & 0,424 \\
\hline & $\beta_{5}$ & $-0,075$ & $-0,075$ & 0,234 & $-0,527$ & 0,378 \\
\hline & $\beta_{6}$ & $-0,016$ & $-0,009$ & 0,026 & $-0,064$ & 0,038 \\
\hline Sem & $\alpha$ & 2,351 & 2,302 & 0,344 & 1,748 & 3,021 \\
\hline Observações & $\lambda$ & $-2,782$ & $-2,654$ & 0,694 & $-4,135$ & $-1,582$ \\
\hline 23,176 e 279 & $\eta$ & 2,752 & 2,454 & 1,594 & 0,163 & 5,837 \\
\hline & $\beta_{0}$ & 1,574 & 1,560 & 0,543 & 0,575 & 2,644 \\
\hline & $\beta_{1}$ & 0,100 & 0,105 & 0,182 & $-0,251$ & 0,465 \\
\hline & $\beta_{2}$ & $-0,010$ & $-0,009$ & 0,006 & $-0,023$ & 0,002 \\
\hline & $\beta_{3}$ & $-0,445$ & $-0,442$ & 0,104 & $-0,645$ & $-0,238$ \\
\hline & $\beta_{4}$ & 0,073 & 0,071 & 0,195 & $-0,325$ & 0,434 \\
\hline & $\beta_{5}$ & $-0,068$ & $-0,071$ & 0,243 & $-0,544$ & 0,416 \\
\hline & $\beta_{6}$ & $-0,012$ & $-0,012$ & 0,028 & $-0,065$ & 0,045 \\
\hline
\end{tabular}


Em seguida, reajustamos o modelo de regressão WBNcr-PA sem as covariáveis não significantes para o conjunto de dados originais e para os dados sem observações 23, 176 e 279 . Note que as médias a posteriori dos parâmetros do modelo de regressão WBNcr-PA não são sensíveis à eliminação das observações influentes, o que pode ser visto na Tabela 4.17.

TABELA 4.17: Resumo a posteriori dos parâmetros do modelo de regressão WBNcr-PA para dados reais com covariável significante

\begin{tabular}{ccccccc}
\hline \hline & & & & & \multicolumn{2}{c}{ Intervalo HPD (95\%) } \\
\cline { 6 - 7 } Dados & Parâmetro & Média & Mediana & EP & LI & LS \\
\hline Original & $\alpha$ & 2,377 & 2,355 & 0,277 & 1,857 & 2,902 \\
& $\lambda$ & $-3,314$ & $-3,156$ & 0,881 & $-4,813$ & $-1,944$ \\
& $\eta$ & 3,714 & 3,585 & 1,391 & 1,210 & 6,482 \\
& $\beta_{0}$ & 0,880 & 0,899 & 0,322 & 0,218 & 1,526 \\
& $\beta_{3}$ & $-0,382$ & $-0,376$ & 0,093 & $-0,570$ & $-0,208$ \\
\hline Sem & $\alpha$ & 2,481 & 2,460 & 0,286 & 1,924 & 3,011 \\
Observações & $\lambda$ & $-3,123$ & $-3,056$ & 0,613 & $-4,392$ & $-2,038$ \\
23, 176 e 279 & $\eta$ & 3,629 & 3,493 & 1,344 & 1,329 & 6,272 \\
& $\beta_{0}$ & 0,977 & 0,965 & 0,256 & 0,514 & 1,493 \\
& $\beta_{3}$ & $-0,390$ & $-0,383$ & 0,090 & $-0,574$ & $-0,223$ \\
\hline
\end{tabular}

As estimativas de Monte Carlo das quatro medidas são apresentadas na Figura 4.8, na qual duas possíveis observações influentes são indicadas pela medida de $\chi^{2}$-divergence, mas não há possível observação influente indicada por outras três medidas. Além disso, o modelo de regressão WBNcr-PA não é sensível sob exclusão das observações influentes.
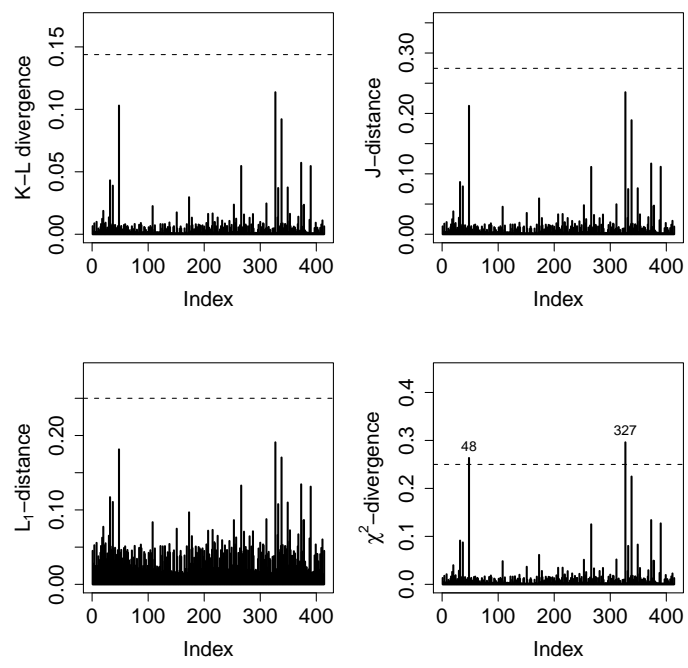

FIGURA 4.8: Estimativas de Monte Carlo das medidas de $\psi$-divergência do modelo de regressão WBNcr-PA ajustado sem covariáveis não significantes e observação 23, 176 e 279. 
TABELA 4.18: Resumo a posteriori das frações de cura de acordo com categoria de nódulo

\begin{tabular}{cccccc}
\hline & & & & \multicolumn{2}{c}{ Intervalo HPD $(95 \%)$} \\
\cline { 5 - 6 } Parâmetro & Média & Mediana & EP & LI & LS \\
\hline$p_{0_{1}}$ & 0,5837 & 0,5927 & 0,0673 & 0,4392 & 0,6972 \\
$p_{0_{2}}$ & 0,5005 & 0,5101 & 0,0557 & 0,3785 & 0,5947 \\
$p_{0_{3}}$ & 0,4165 & 0,4212 & 0,0471 & 0,3194 & 0,5020 \\
$p_{0_{4}}$ & 0,3371 & 0,3375 & 0,0452 & 0,2423 & 0,4172 \\
\hline
\end{tabular}

(a)

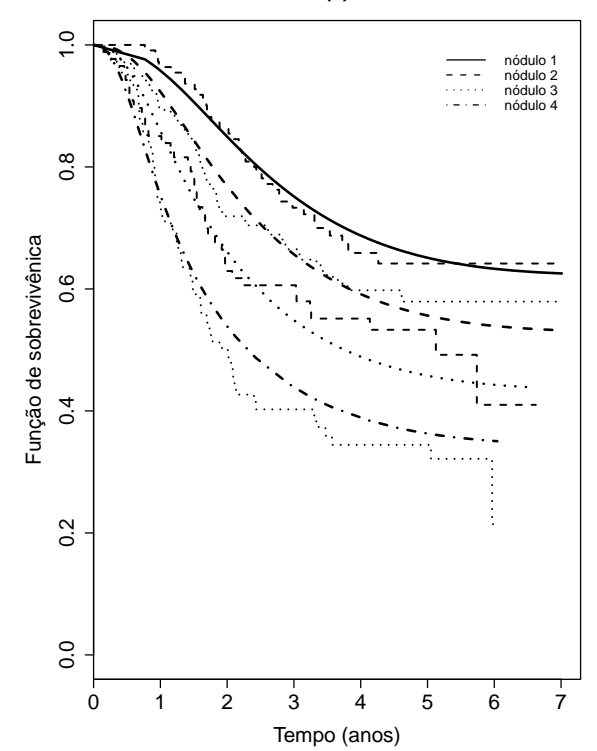

(b)

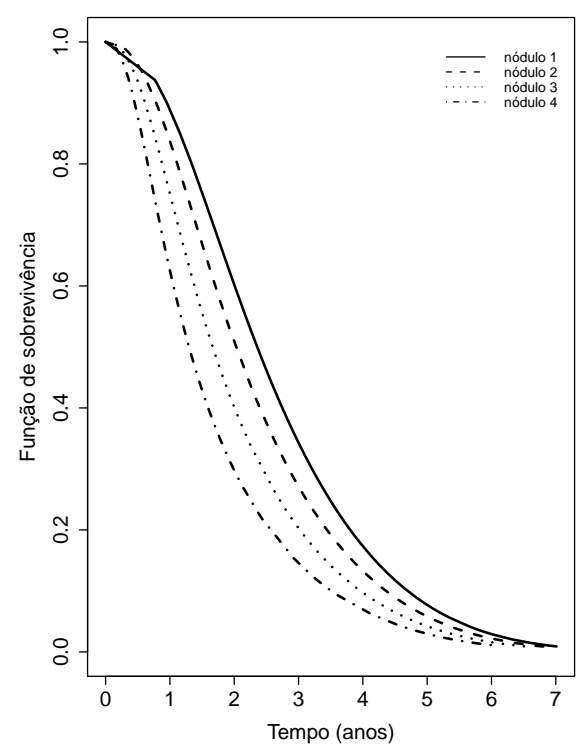

FIGURA 4.9: (a): Estimativas de Kaplan-Meier e paramétrica da função de sobrevivência do modelo de regressão WBNcr-PA de acordo com a categoria de nódulo (1-4) e (b): Funções de sobrevivência ajustadas estratificadas por nódulo para o paciente sem fração de cura

Como a covariável de categoria de nódulo é significante na Tabela 4.17, então existe uma relação com a classificação do tumor, sendo que a fração de cura diminui em indivíduos com maior categoria de nódulo. A Figura 4.9 (a) mostra que o modelo fornece uma boa aproximação das estimativas de Kaplan-Meier da função de sobrevivência e as funções de sobrevivência estratificadas por categoria de nódulo para pacientes sem curas são apresentadas na figura (b), o que mostrou que os pacientes sem fração de cura, com qualquer categoria de nódulo, tem baixa chance de sobreviver após sete anos. 


\subsection{Conclusão}

Neste capítulo de trabalho propomos o modelo de regressão WBN com fração de cura sobre diferentes mecanismos de ativação para ajustar os dados de tempo de vida. Na análise clássica, o estimador de máxima verossimilhança foi usado para estimar os parâmetros dos modelos propostos, que foram comparados com as distribuições Weibull Geométrica e Weibull Poisson como casos particulares. Na abordagem Bayesiana o método de cadeia Markov Monte Carlo (MCMC) foi usado para determinar as médias a posteriori dos parâmetros dos modelos propostos; além disso, a análise de influência caso a caso foi baseada nas medidas de $K$ - $L$ divergence, $L_{1}$ distance, J-distance e $\chi^{2}$-divergence, com o objetivo de estudar a sensibilidade das médias a posteriori dos parâmetros dos modelos sobre as perturbações no modelo/dados.

Finalmente, um conjunto de dados real foi ajustado pelo modelo de regressão WBNcr para mostrar a potencialidade das metodologias; observe-se que o modelo de regressão WBN com fração de cura sobre primeiro esquema de ativação fornece o melhor ajuste nas ambas abordagens. A probabilidade de sobrevivência dos pacientes diminui rapidamente conforme seja maior o categoria do nódulo. Além disso, a função de sobrevivência dos pacientes sem fração de cura também pode ser estimada para comparar com o tratamento, e a interpretação do papel da covariáveis é fácil devido à parametrização considerada na fração de cura. 


\section{Capítulo 5}

\section{Considerações finais}

Neste trabalho, propomos a distribuição WBN sobre uma estrutura de ativação latente para explicar a ocorrência do evento de interesse, em que o número de causas competitivas foi modelado pela distribuição Binomial Negativa, considerando que as causas competitivas assumem os mecanismos de primeira ativação, segunda ativação e ativação aleatória. A distribuição proposta generalizou várias famílias de distribuição; além disso, sua flexibilidade é facilmente observada por sua função de risco, pois possui várias formas não monótonas. Várias propriedades matemáticas padrões da distribuição foram obtidas e os momentos apresentaram forma fechada para alguns casos especiais. Para estimar os parâmetros desses modelos foi utilizado o método de máxima verossimilhança e os critérios AIC e BSC, e a teste de RV foram utilizadas na comparação dos modelos.

O modelo de regressão Log-WBN foi proposto baseando-se na mesma estrutura para os casos em que os eventos de interesse são afetados pelas covariáveis. Os métodos de máxima verosimilhança e Monte Carlo via Cadeias de Markov (MCMC) foram aplicados para desenvolver os procedimentos inferenciais em uma perspectiva clássica e Bayesiana, respectivamente. De acordo com os resultados de simulação, os modelos assumiram os três mecanismos de ativação propostos, e puderam ser discriminados nos ajustes dos modelos para um conjunto de dados. Além disso, no estudo de análise de influência caso a caso, as quatro medidas de divergência propostas, $K$-L divergence, $L_{1}$-distance, J-distance e $\chi^{2}$-divergence, apresentaram bom desempenho para identificar as observações influentes, em que $\chi^{2}$-divergência foi mais sensível do que outras medidas, o que pudemos comprovar por meio dos estudos de simulação e da aplicação para os dados reais.

Finalmente, foram definidos os modelos de regressão para os dados com fração de cura, 
baseando-se na mesma estrutura de ativação latente e os mesmos estudos foram realizados do modelo de regressão Log-WBN. A simulação e aplicação mostraram que os modelos ajustam adequadamente os dados de sobrevivência, e as médias a posteriori dos parâmetros do modelo de regressão WBN com fração de cura não são sensíveis sobre eliminação das observações influentes. Além disso, a função de sobrevivência dos modelos sem fração de cura também pode ser estimada para comparar com o tratamento, e a interpretação do papel das covariáveis é fácil devido à parametrização considerada na fração de cura.

Como propostas futuras podem-se considerar os seguintes temas de pesquisa:

1. Realizar um estudo de diagnóstico embasado na abordagem clássica, utilizando a técnica de influência local proposta por Cook (1986) para os modelos propostos, tendo como base a estrutura de ativação latente.

2. Propor um novo modelo considerando uma estrutura de ativação latente para explicar a ocorrência do evento de interesse, em que o número de causas competitivas é modelado pela distribuição Binomial Negativa e os tempos não observados devido às causas seguem a distribuição Gama generalizada (Lawless, 2003).

3. Propor um modelo para dados de sobrevivência multivariados com presença de fração de cura, considerando os modelos com ponto de mudança na função de risco (Achcar \& Bolfarine, 1989) em um cenário de riscos latentes. 


\section{Referências Bibliográficas}

Achcar, J. A. \& Bolfarine, H. (1989). Constant hazard against a change-point alternative: a bayesian approach with censored data. Communications in Statistics. Theory and Methods, 18(10).

Adamidis, K. \& Loukas, S. (1998). A lifetime distribution with decreasing failure rate. Statistics $\&$ Probability Letters, 39(1), 35-42.

Adamidis, K., Dimitrakopoulou, T. \& Loukas, S. (2005). On a generalization of the exponentialgeometric distribution. Statist. Probab. Lett., 73, 259-269.

Barreto-Souza, W., Santos, A. \& Cordeiro, M. G. (2010). The beta generalized exponential distribution. Journal of Statistical Computation and Simulation, 80(2), 159-172.

Barreto-Souza, W., De Morais, A. \& Cordeiro, G. (2011). The Weibull-Geometric distribution. Journal of Statistical Computation and Simulation, to appear, 81(5), 645-657.

Basu, A. \& Klein, J. (1982). Some recent development in competing risks theory, volume 2. Proceedings on Survival Analysis.

Berkson, J. \& Gage, R. P. (1952). Survival cure for cancer patients following treatment. Journal of the American Statistical Association, 47, 501-515.

Boag, J. (1949). Maximum likelihood estimates of the proportion of patients cured by cancer therapy. Journal of the Royal Statistical Society. Series B, 11, 15-53.

Brooks, S. P. (2002). Discussion on the paper by Spiegelhalter, Best, Carlin, and van der Linde (2002). Journal of the Royal Statistical Society B, 64, 616-618.

Cancho, V., Ortega, E. \& Bolfarine, H. (2009). The Log-exponentiated-Weibull Regression Models with Cure Rate: Local Influence and Residual Analysis. Journal of Data Science, 7, 433-458.

Cancho, V., Ortega, E. M. M. \& Paula, G. (2010). On estimation and influence diagnostics for log-birnbaum-saunders student-t regression models: Full bayesian analysis. Journal of Statistical Planning and Inference, 140, 2486-2496.

Cancho, V. G., Rodrigues, J. \& Castro, M. (2011). A flexive model for survival with a cure rate: A bayesian approach. Journal of Applied Statistics, 38, 57-70.

Carlin, B. P. \& Louis, T. A. (2001). Bayes and Empirical Bayes Methods for Data Analysis. Chapman \& Hall/CRC, Boca Raton, second edition.

Chen, M. \& Shao, Q. (1998). Monte Carlo Estimation of Bayesian Credible and HPD Intervals. Journal of Computational and Graphical Satatistics, pages 69-92. 
Chen, M. H., Ibrahim, J. G. \& Sinha, D. (1999). A new bayesian model for survival data with a surviving fraction. Journal of the American Statistical Association, 94, 909-919.

Chen, M. H., Shao, Q. M. \& Ibrahim, J. G. (2000). Monte Carlo methods in Bayesian computation. Springer-Verlag, New York.

Cho, H., Ibrahim, J. G., Sinha, D. \& Shu, H. (2009). Bayesian case influence diagnostics for survival models. Biometrics, 65, 116-124.

Colosimo, E. A. \& Giolo, S. R. (2006). Análise de Sobrevivência Aplicada. Editora Edgard Blücher, Brasil.

Cook, R. \& Weisberg, S. (1982). Residuals and influence in regression. Chapman and Hall New York.

Cook, R. D. (1977). Detection of infuential observations in linear regression. Technometrics, $19,15-18$.

Cook, R. D. (1986). Assessment of local influence. Journal of the Royal Statistical Society. Series B (Methodological), 48, 133-169.

Cooner, F., Banerjee, S. \& M., M. A. (2006). Modelling geographically referenced survival data with a cure fraction. Statistic Methods in Medical Research, 15, 307-324.

Cooner, F., Banerjee, S., Carlin, B. \& Sinha, D. (2007). Flexible cure rate modeling under latent activation schemes. Journal of the American Statistical Association, 102, 560-572.

Cordeiro, G. M. \& Castro, M. (2011). A new family of generalized distribution. Journal of Statistical Computation and Simulation, 81(7), 883-898.

Dey, D. \& Birmiwal, L. (1994). Robust bayesian analysis using divergence measures. Statistics \& Probability Letters, 20(4), 287-294.

Dunn, P. K. \& Smyth, G. K. (1996). Randomized quantile residuals. Journal of Computational and Graphical Statistic, 5(3), 236-224.

Eugene, N., C., L. \& Famoye, F. (2002). Beta-normal distribution and its appplications. Commum. in Stat.-Theory and Methods, 31(4), 497-512.

Famoye, F., Lee, C. \& Olumolade, O. (2005). The beta-weibull distribution. J. Statistic Theory and Application, 4, 121-136.

G., C. V., F., L. \& Barriga, D. C. G. (2011). The poisson-exponential lifetime distribution. Computational Statistic and Data Analysis, 55(1), 677-686.

Gamerman, D. \& Lopes, H. F. (2006). Markov Chain Monte Carlo: Stochastic Simulation for Bayesian Inference. Chapman \& Hall/CRC, Boca Raton, second edition.

Geisser, S. \& Eddy, W. (1979). A predictive approach to model selection. Journal of the American Statistical Association, pages 153-160.

Gelfand, A., Dey, D. \& Chang, H. (1992). Model determination using predictive distributions with implementation via sampling based methods (with discussion). bayesian statistics 4 . eds: J. bernardo et al. BernardoJM, BergerJO, Dawid A P, Sm ith A FM(editors). OxfordUniversityPress, 1(4), 7-167.

Geweke, J. (1992). Evaluating the accuracy of sampling-based approaches to calculating posterior moments. Clarendon Press, Oxford, UK. 
Gradshteyn, I. S. \& Ryzhik, I. M. (2000). Table of Integrals, Series, and Products. Academic Press, San Diego.

Ibrahim, J., Chen, M. \& Sinha, D. (2001). Bayesian survival analysis. Springer Verlag.

Kaplan, E. L. \& Meier, P. (1958). Nonparametric estimation from incomplete observations. Journal of the American Statistical Association, 53.

Kus, C. (2007). A new lifetime distribution. Computational Statistics \& Data analysis, 51(9), $4497-4509$.

Labra, F. V., Aoki, R. \& Bolfarine, H. (2005). Local influence in null intercept measurement error regression under a student-t model. Journal of Applied Statistics, 32, 723-740.

Lai, C., Xie, M. \& Murthy, D. (2003). A modified Weibull distribution. IEEE Transactions on reliability, 52(1), 33-37.

Lawless, J. F. (2003). Statistical models and methods for lifetime data. Wiley series in probability and statistics. John Wiley \& Sons Inc., Hoboken, NJ, second edition. ISBN 0-471-37215-3.

Lopes, C., Mendes, C. \& Bolfarine, H. (2012). Random effects in promotion time cure rate models. Computational Statistics and Data Analysis, 56, 75-87.

Maller, R. A. \& Zhou, X. (1996). Survival Analysis with Long-Term Survivors. Wiley, New York.

Mudholkar, G. \& Srivastava, D. (1993). Exponentiated Weibull family for analyzing bathtub failure-ratedata. IEEE Transactions on Reliability, 42(2), 299-302.

Mudholkar, G. S., Srivastava, D. K. \& Kollia, G. D. (1996). A generalization of the weibull distribution with application to the analysis of survival data. Journal of the American Statistical Association, 91(436), 1575-1583.

Nelson, W. (1990). Accelerated testing-statistical models, test plans and data analysis. John Wiley \& Sons Inc., New York. ISBN 0-471-09458-7. Wiley Series in Probability and Mathematical Statistics.

Ortega, E. M. M., Bolfarine, H. \& Paula, G. (2003). Influence diagnostics in generalized loggamma regression models. Computational Statistics \& Data Analysis, 42, 165-186.

Ortega, E. M. M., Cancho, V. G. \& Bolfarine, H. (2006). Influence diagnostics in exponentiatedWeibull regression models with censored data. Statistics and Operations Research Transactions, 30, 171-192.

Peng, F. \& Dey, D. (1995). Bayesian analysis of outlier problems using divergence measures. Canadian Journal of Statistics, 23(2), 199-213.

Piegorsch, W. W. (1990). Maximum likelihood estimation for the negative binomial dispersion parameter. Biometrics, 46, 863-867.

R Development Core Team (2010). R: A Language and Environment for Statistical Computing. R Foundation for Statistical Computing, Vienna, Austria.

Rodrigues, J., Cancho, V. G. \& Castro, M. (2008). Teoria Unificada de Análise de Sobrevivência. ABE, Brasil.

Rodrigues, J., Cancho, V., Castro, M. \& Louzada-Neto, F. (2009). On the unification of longterm survival models. Statistics and Probability Letters, 79, 753-759. 
Rodrigues, J., Cancho, V. G., Castro, M. \& Balakrishnan, N. (2010a). A Bayesian destructive weighted Poisson cure rate model and an application to a cutaneous melanoma data. Statistical Methods in Medical Research.

Rodrigues, J., Castro, M., Balakrishnan, N. \& Cancho, V. (2010b). Destructive weighted Poisson cure rate models. Lifetime Data Analysis.

Roman, M., Louzada Neto, F. \& G., C. V. (2011). The complementary exponential geometric distribution: Model, properties, and a comparison with its counterpart. Computational Statistic E Data Analysis, 55, 2516-2524.

Silva, G. O., Ortega, E. M. M. \& M., C. G. (2010). The beta modified weibull distribution. Lifetime Data Analysis, 16(3), 409-430.

Smith, R. L. \& Naylor, J. C. (1987). A comparison of maximum likelihood and bayesian estimatores for the three-parameter weibull distribution. Appl. Stat., 36(3), 358-369.

Spiegelhalter, D. J., Best, N. G., Carlin, B. P. \& van der Linde, A. (2002). Bayesian measures of model complexity and fit. Journal of the Royal Statistical Society B, 64, 583-639.

Tournoud, M. \& Ecochard, R. (2007). Application of the promotion time cure model with time-changing exposure to the study of HIV/AIDS and other infectious diseases. Statistics in medicine, 26, 1008-1021.

Tsodikov, A. D., Ibrahim, J. G. \& Yakovlev, A. Y. (2003). Estimating cure rates from survival data: an alternative to two-component mixture models. Journal of the American Statistical Association, 98, 1063-1078.

Weiss, R. (1996). An approach to bayesian sensitivity analysis. Journal of the Royal Statistical Society. Series B (Methodological), pages 739-750.

Xie, M., Tang, Y. \& Goh, T. N. (2002). A modified weibull extension with bathtub-shaped failure rate function. Realiability Engineering and System Safety, 76(3), 279-285.

Yakovlev, A. Y. \& Tsodikov, A. D. (1996). Stochastic Models of Tumor Latency and Their Biostatistical Applications. World Scientific, New Jersey. 